\title{
Observational hints of radial migration in disc galaxies from CALIFA ${ }^{\star}, \star \star$
}

T. Ruiz-Lara ${ }^{1,2,3,4}$, I. Pérez ${ }^{1,2}$, E. Florido ${ }^{1,2}$, P. Sánchez-Blázquez ${ }^{5}$, J. Méndez-Abreu ${ }^{3,4,6}$, L. Sánchez-Menguiano ${ }^{7,1}$, S. F. Sánchez ${ }^{8}$, M. Lyubenova ${ }^{9}$, J. Falcón-Barroso ${ }^{3,4}$, G. van de Ven $^{10}$, R. A. Marino ${ }^{11}$, A. de Lorenzo-Cáceres ${ }^{8}$, C. Catalán-Torrecilla ${ }^{12}$, L. Costantin ${ }^{13}$, J. Bland-Hawthorn ${ }^{14}$, L. Galbany ${ }^{15}$, R. García-Benito ${ }^{7}$, B. Husemann ${ }^{10}$, C. Kehrig ${ }^{7}$, I. Márquez ${ }^{7}$, D. Mast ${ }^{16,17}$, C. J. Walcher ${ }^{18}$, S. Zibetti ${ }^{19}$, B. Ziegler ${ }^{20}$, and the CALIFA team

1 Departamento de Física Teórica y del Cosmos, Universidad de Granada, Campus de Fuentenueva, 18071 Granada, Spain e-mail: tomasruizlara@gmail.com

2 Instituto Carlos I de Física Teórica y Computacional, Universidad de Granada, 18071 Granada, Spain

3 Instituto de Astrofísica de Canarias, Calle Vía Láctea s/n, 38205 La Laguna, Tenerife, Spain

${ }^{4}$ Departamento de Astrofísica, Universidad de La Laguna, 38200 La Laguna, Tenerife, Spain

5 Departamento de Física Teórica, Universidad Autónoma de Madrid, 28049 Cantoblanco, Spain

${ }^{6}$ School of Physics and Astronomy, University of St Andrews, SUPA, North Haugh, KY16 9SS St Andrews, UK

7 Instituto de Astrofísica de Andalucía (CSIC), Glorieta de la Astronomía s/n, Aptdo. 3004, 18080 Granada, Spain

8 Instituto de Astronomía, Universidad Nacional Autónoma de México, A.P. 70-264, 04510 México D.F., Mexico

9 Kapteyn Astronomical Institute, University of Groningen, PO Box 800, 9700 AV Groningen, The Netherlands

10 Max-Planck-Institut für Astronomie, Königstuhl 17, 69117 Heidelberg, Germany

11 Department of Physics, Institute for Astronomy, ETH Zürich, 8093 Zürich, Switzerland

12 Departamento de Astrofísica y CC. de la Atmósfera, Universidad Complutense de Madrid, 28040 Madrid, Spain

13 Dipartimento di Fisica e Astronomia G. Galilei, Università di Padova, vicolo dell'Osservatorio 3, 35122 Padova, Italy

14 Sydney Institute for Astronomy, School of Physics A28, University of Sydney, Sydney, NSW 2006, Australia

15 PITT PACC, Department of Physics and Astronomy, University of Pittsburgh, Pittsburgh, PA 15260, USA

16 Observatorio Astronómico, Laprida 854, 5000BGR Córdoba, Argentina

17 Consejo de Investigaciones Científicas y Técnicas de la República Argentina, Avda. Rivadavia 1917, 1033AAJ CABA, Argentina

18 Leibniz-Institut für Astrophysik Potsdam (AIP), An der Sternwarte 16, 14482 Potsdam, Germany

19 INAF-Osservatorio Astrofisico di Arcetri, Largo Enrico Fermi 5, 50125 Firenze, Italy

20 University of Vienna, Department of Astrophysics, Türkenschanzstrasse 17, 1180 Vienna, Austria

Received 27 February 2017 / Accepted 3 May 2017

\section{ABSTRACT}

\begin{abstract}
Context. According to numerical simulations, stars are not always kept at their birth galactocentric distances but they have a tendency to migrate. The importance of this radial migration in shaping galactic light distributions is still unclear. However, if radial migration is indeed important, galaxies with different surface brightness (SB) profiles must display differences in their stellar population properties. Aims. We investigate the role of radial migration in the light distribution and radial stellar content by comparing the inner colour, age, and metallicity gradients for galaxies with different SB profiles. We define these inner parts, avoiding the bulge and bar regions and up to around three disc scale lengths (type I, pure exponential) or the break radius (type II, downbending; type III, upbending).

Methods. We analysed 214 spiral galaxies from the CALIFA survey covering different SB profiles. We made use of GASP2D and SDSS data to characterise the light distribution and obtain colour profiles of these spiral galaxies. The stellar age and metallicity profiles were computed using a methodology based on full-spectrum fitting techniques (pPXF, GANDALF, and STECKMAP) to the Integral Field Spectroscopic CALIFA data.

Results. The distributions of the colour, stellar age, and stellar metallicity gradients in the inner parts for galaxies displaying different SB profiles are unalike as suggested by Kolmogorov-Smirnov and Anderson-Darling tests. We find a trend in which type II galaxies show the steepest profiles of all, type III show the shallowest, and type I display an intermediate behaviour.

Conclusions. These results are consistent with a scenario in which radial migration is more efficient for type III galaxies than for type I systems, where type II galaxies present the lowest radial migration efficiency. In such a scenario, radial migration mixes the stellar content, thereby flattening the radial stellar properties and shaping different SB profiles. However, in light of these results we cannot further quantify the importance of radial migration in shaping spiral galaxies, and other processes, such as recent star formation or satellite accretion, might play a role.
\end{abstract}

Key words. galaxies: stellar content - galaxies: spiral - galaxies: evolution - galaxies: formation - galaxies: structure

\footnotetext{
* Based on observations collected at the Centro Astronómico Hispano Alemán (CAHA) at Calar Alto, operated jointly by the MaxPlanck Institut für Astronomie and the Instituto de Astrofísica de Andalucía (CSIC).

$\star \star$ Table 1 is only available at the CDS via anonymous ftp to cdsarc.u-strasbg.fr (130.79.128.5) or via

http://cdsarc.u-strasbg.fr/viz-bin/qcat?J/A+A/604/A4
}

\section{Introduction}

The analysis of the stellar content in galaxies is an essential tool to unveil the processes that these systems underwent throughout their history (e.g. MacArthur et al. 2009; Roediger et al. 2011a; Sánchez-Blázquez et al. 2011; Pérez \& Sánchez-Blázquez 2011; Pérez et al. 2013). Stellar populations reflect the chemistry of 
the interstellar medium at the moment of their formation and temporal variations in star formation activity can give us essential information about the history of the galaxy (Cole et al 2007; Gallart et al. 2015; González Delgado et al. 2015, 2016; Beasley et al. 2015). However, galaxies are dynamical systems in continuous change and stars do not remain at their birth locations. We must take into account the effect of stellar motions to properly interpret stellar population information (e.g. Roškar et al. 2008b; Martínez-Serrano et al. 2009).

Several theoretical works have studied why stars undergo radial motions and their effects on disc properties (Sellwood \& Binney 2002; Debattista et al. 2006; Younger et al. 2007; Roškar et al. 2008a,b; Sánchez-Blázquez et al. 2009; Minchev \& Famaey 2010; Minchev et al. 2011, 2012b; Roškar et al. 2012; Bird et al. 2012). The causes for such motions can be attributed to internal (mainly caused by axisymmetric structures) or external (effect of satellite influence) agents. Sellwood \& Binney (2002) proposed that stars close to the corotation resonance of transient spirals experience large changes in their radial positions. In addition, a non-linear coupling of non-axisymmetric structures such as the bar and the spiral structure leads to stronger migrations to those caused by the single presence of transient spirals (Minchev \& Famaey 2010; Minchev et al. $2012 b, a)$. The influence of nearby satellites, as well as satellite accretion, can also induce mixing in stellar discs (Younger et al. 2007; Bird et al. 2012).

Recent simulations have suggested that these radial motions have a considerable effect on the galaxy properties. Roškar et al. (2008a), using $N$-body and smoothed-particle hydrodynamic (SPH) simulations of an isolated and idealised disc, found mass profiles with a lack of mass in the outer parts and age profiles with a characteristic "U-shape" (i.e. an age radial decline followed by an outer upturn). These authors suggested that these features found in the outer parts (i.e. lack of mass and outer ageing) are attributed to the interplay between a radial star formation cut-off and radial redistribution of stars induced by transient spiral arms. Sánchez-Blázquez et al. (2009), using fully cosmological hydrodynamical simulations, also found a downbending light surface density profile (lack of light in the outer parts) and a "U-shape" age profile in their simulated disc. In this case, the authors claimed that breaks in the light distribution do not necessarily correspond to breaks in the mass distribution, which suggests that the break origin is linked to two main processes: first, a radial change in the slope of the star formation profile linked to a drop in the gas density from a warp as the main cause and, second, radial migration of stars towards larger radii. Such "U-shaped" age gradient was found even in the absence of radial migration. Although theoretical works have concentrated on galaxies displaying downbending profiles (type II), several observational works have also observed systems with pure exponential (type I) declines of the light profiles and upbending (type III) surface brightness (SB) distributions (e.g. Bland-Hawthorn et al. 2005; Pohlen \& Trujillo 2006; Erwin et al. 2008; Gutiérrez et al. 2011; Marino et al. 2016).

Although there is no clear consensus on the causes of radial migration or a definite explanation for the simulated SB and age profiles, all these works point towards an important amount of stars migrating from the inner to outer regions. In particular, Sánchez-Blázquez et al. (2009) found that $57 \%$ of the stars currently located in the outer parts of their cosmologically simulated disc came from the inner region and had mean values of the traversed radial distance of $\sim 3.4 \mathrm{kpc}$. Other works have also analysed the amount of stellar particles populating the outer regions and coming from the inner regions.
Roškar et al. (2008b) found that the percentage of outwards migrating stars is up to $\sim 85 \%$ with the average change in radius being $3.7 \mathrm{kpc}$, while Martínez-Serrano et al. (2009) obtained a percentage as high as $64-78 \%$ in their cosmological discs. Roškar et al. (2012) analysed in detail the origin of the radial migration observed in their idealised and isolated discs and obtained that nearly $50 \%$ of the stars populating their solar neighbourhood $(7<R[\mathrm{kpc}]<9)$ came from the inner disc; some of these experienced radial changes as high as $7 \mathrm{kpc}$ although this is not the norm. Observationally, evidence of radial migration has also been found in stars in the Milky Way (Feltzing et al. 2001; Nordström et al. 2004; Bergemann et al. 2014). In particular, the RAVE collaboration ("the Radial Velocity Experiment", Steinmetz et al. 2006; Kordopatis et al. 2013) have recently found that around half of the supersolar metallicity stars currently located in the solar neighbourhood have migrated from inner regions (Kordopatis et al. 2015). This radial redistribution of material should affect, not only the outer parts, but the overall stellar population and light distributions, especially if the number of migrating stars is high.

In particular, in Sánchez-Blázquez et al. (2009), where the broken profiles were caused by a combination of a radial change in the star formation rate linked to a warp and radial migration, the authors speculated that the different observed SB profiles in the literature might be explained by different combinations of both processes. If we ignore the effect of the warp, we would expect a smooth change in the star formation rate per area unit leading to a pure exponential profile. Depending on the intensity of the radial migration, we could change from galaxies displaying a type II surface density profile (systems with little outwards radial migration) to galaxies displaying a type III profile (with a higher efficiency of the outwards radial migration), where type I galaxies are a case in between. Thus, different radial migration efficiencies might produce different SB profiles. If this scenario proposed by Sánchez-Blázquez et al. (2009) is correct, then, galaxies displaying different SB distributions must present differences in the stellar content throughout their discs. Therefore, the analysis of the stellar populations from the inner regions up to the outer discs of spiral galaxies is essential to understand better the role of radial migration, the general assembly of spiral galaxies, and to refine and constrain galaxy formation models. This analysis can be carried out following different approaches.

One possible approach consists of taking photometric images using different broadband filters. As a first approximation, light differences from different filters (colours) can be interpreted as variations in the properties of the stellar content. A vast amount of works have used this approach to determine stellar population gradients (e.g. Peletier 1993; de Jong 1996; Peletier \& Balcells 1996; Jansen et al. 2000; Bell \& de Jong 2000; MacArthur et al. 2004; Muñoz-Mateos et al. 2009; Roediger et al. 2011b,a, 2012). Whether optical colours can be interpreted as a proxy for stellar age or metallicity is still controversial.

Another approach to analyse stellar light uses spectroscopic data. This approach allows us to study specific features that are dependent on the stellar age and metallicity, i.e. the line-strength indices (e.g. Rose 1984; Faber et al. 1985; Bica \& Alloin 1986a; Bica \& Alloin 1986b; Bica 1988; Gorgas et al. 1993; Worthey 1994; Bica et al. 1994; Vazdekis et al. 1996; Worthey \& Ottaviani 1997; Kauffmann et al. 2003). Indices have been used to obtain single stellar population (SSP) equivalent values for age and metallicity in "simple" systems, such as globular clusters or elliptical galaxies (e.g. Peletier et al. 2007; Kuntschner et al. 2010). However, in the recent years great effort has made towards improving the quality of the information recovered from 

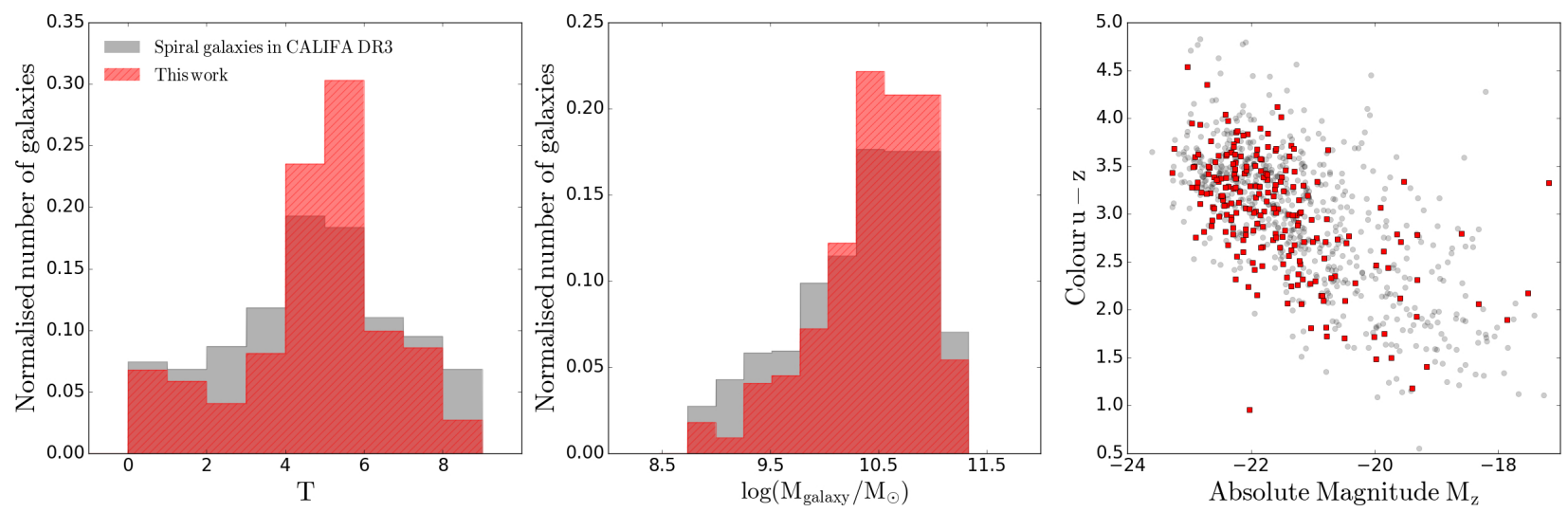

Fig. 1. Characterisation of the sample of galaxies under analysis in this work (red) compared to the CALIFA DR3 sample of galaxies limited to spiral systems (grey). Left-hand panel: distribution is indicated of the morphological types according to the $T$ parameter from Walcher et al. (2014). Middle panel: distribution is indicated of stellar masses from Walcher et al. (2014). Right-hand panel: distribution is indicated of the galaxies in the $(u-z)-M_{z}$ colour magnitude diagram.

indices and making possible the application of this approach to any kind of system (e.g. Gallazzi et al. 2005, 2006, 2008). The combination of recently developed Bayesian approaches, improved spectral libraries, and high-quality observed spectra has allowed studies based on indices to obtain star formation histories as well as metallicity and dust distributions (Zibetti et al. 2017). However, the needed signal-to-noise (S/N) hampers this kind of analysis in regions of low SB or with data of limited quality.

In order to overcome this issue and to maximise the information used from observed spectra, several codes have been developed in the recent years analysing wide wavelength ranges (e.g. Heavens et al. 2000; Reichardt et al. 2001; Cid Fernandes et al. 2005; Tojeiro et al. 2007; Ocvirk et al. 2006b,a; Koleva et al. 2009). This full-spectrum fitting approach has been proven successful at further minimising the well-known age-metallicity degeneracy (Sánchez-Blázquez et al. 2011). However, studies of the stellar content on spiral galaxies are still very scarce, limited to the bulges or the inner discs (MacArthur et al. 2009; Sánchez-Blázquez et al. 2011, 2014a; Sánchez et al. 2011; Morelli et al. 2015, 2016).

The emergence of integral field spectroscopy (IFS) makes a reliable analysis of the stellar content up to larger galactocentric distances possible. The IFS instruments and data at our disposal allow us to carry out new stellar population studies with unprecedented quality (Yoachim et al. 2012; Sánchez-Blázquez et al. 2014b; González Delgado et al. 2015; Ruiz-Lara et al. 2016b).

In this paper we study the stellar content of a sample of 214 spiral galaxies from the CALIFA survey ("Calar Alto Legacy Integral Field spectroscopy Area survey” Sánchez et al. 2012) using full-spectrum fitting techniques to assess, for the first time in such a large sample, the role of radial migration in shaping the properties that we observe in spiral galaxies. In addition, we analyse photometric data from the Sloan Digital Sky Survey (SDSS; York et al. 2000) to characterise the twodimensional (2D) light distribution of these galaxies. This analysis allows us to test if galaxies with the same SB profiles display similarities in their inner stellar content. Besides, we also obtain SDSS colour profiles $(g-r, g-i$, and $r-i)$ for a further comparison. In Sect. 2 we define the sample of galaxies under study. We explain our method of analysing SDSS and CALIFA data in Sects. 3 and 4. The main results, discussion, and conclusions of this work are given in Sects. 5-7.

\section{Sample selection}

The sample of galaxies under analysis in this work was chosen from the CALIFA mother sample (Walcher et al. 2014) and the CALIFA extension presented in the CALIFA third data release (DR3; Sánchez et al. 2016). We selected a subset of disc galaxies ( $\mathrm{S} 0 / \mathrm{a}$ to $\mathrm{Sd}$ ) with no signs of interaction according to the sample characterisation presented in Walcher et al. (2014). In addition, the galaxies under analysis are those analysed in Méndez-Abreu et al. (2017) for which accurate 2D decomposition of their light distribution is available. Galaxies fulfilling all these criteria but with inclinations that are larger than $70^{\circ}$ or for which the 2D light decomposition does not find any disc component (those dominated by a spheroid component) are discarded.

The final sample comprises 214 galaxies (124 barred galaxies, 58\%; and 90 unbarred galaxies, $42 \%$ ). Figure 1 is aimed at characterising the morphological and mass distributions and the position in a $(u-z)-M_{z}$ colour magnitude diagram of the selected galaxies (red). In order to compare with the general behaviour of the CALIFA DR3 spiral galaxies, the characteristics of this set of spiral systems are also shown (grey). We can highlight that the galaxies analysed in this study are representative of the CALIFA sample with the addition of the galaxies from the extension projects (not necessarily fulfilling all the CALIFA sample criteria) and thus, unbiased to any particular mass value or morphological type. Table 1 lists the main characteristics of each individual galaxy.

\section{Photometric analysis}

We use the fully calibrated $g, r$, and $i$ band images from the SDSS seventh data release (DR7; Abazajian et al. 2009) to analyse the light distribution of the sample of galaxies. We chose these filters to take advantage of the higher quality of the SDSS images in these bands compared to $u$ and $z$ bands. This analysis has been presented in detail in Méndez-Abreu et al. (2017) covering the entire CALIFA sample. In this section we outline the main characteristics of this analysis and focus on the extraction of colour profiles as a by-product of that work. Méndez-Abreu et al. (2017) provides further detail on the DR7 SDSS photometric images, the general procedure and the sky subtraction, which is critical in the outermost regions of spiral galaxies. 

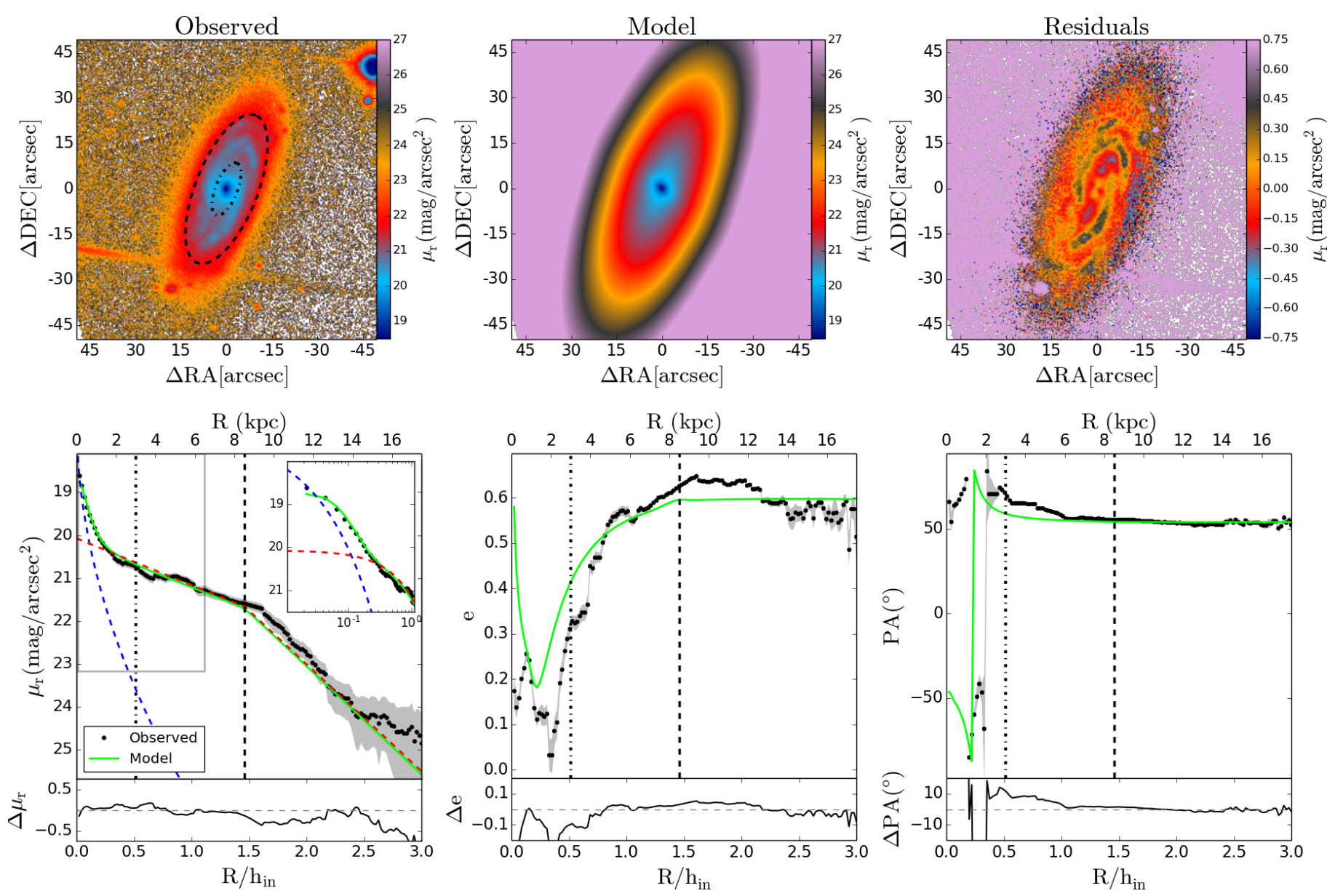

Fig. 2. Plots summarising the 2D decomposition for IC 1199. Top left: $r$-band SDSS image is shown. Top middle: galaxy model derived from GASP2D fit considering a bulge and a broken disc component is shown. Top right: residuals image derived by subtracting the observed image to the model is shown. North is up in all these images. Bottom left: the SDSS $r$-band surface brightness profile is shown. Bottom middle: the ellipticity profile is shown. Bottom right: the position angle profile is shown. In bottom panels: points represent the observed magnitude; shadow areas account for the errors, computed as one sigma of the distribution of values within the ellipse; red dashed line is the broken disc model; blue dashed line follows the bulge light distribution; light green line is the output of ellipse applied to the model; and the inset in bottom left panel is focused on the inner part with a logarithmic radial scale. All bottom panel plots show an auxiliary plot below with the residuals between observed and model values. Dot-dashed vertical lines and the dot-dashed ellipse delimit the bulge-dominated region. Dashed vertical lines and the dashed ellipse are located at the break radius.

\subsection{Two-dimensional surface brightness distribution}

We perform a 2D photometric decomposition of the structural components shaping our galaxy sample by applying GASP2D (Méndez-Abreu et al. 2008, 2014) to the SDSS skysubtracted images. GASP2D iteratively fits a model of the SB distribution to the pixels of the galaxy image by means of a nonlinear least-squares minimisation based on a robust LevenbergMarquardt method (Moré et al. 1980) using the MPFIT algorithm (Markwardt 2009). GASP2D weights every pixel in the image according to the variance of its photon counts, assuming a photon noise limitation and considering the detector readout noise. It deals with seeing effects by convolving the model image with a circular Moffat (Trujillo et al. 2001) point spread function (PSF) with the full width at half maximum (FWHM) measured directly from stars in the galaxy image. The code allows us to determine the different photometric structures contributing to the light distribution of the studied galaxy. The components that GASP2D can fit are a bulge (or nuclear source for very small bulges), a disc (or broken disc), and/or a bar (or double bar). This way, GASP2D provides us with the set of structural parameters of these components that better fit the observed light distribution, such as ellipticities and position angles ( $e$ and PA), the bar length, the break radius $\left(R_{\text {break }}\right)$ for galaxies with broken profiles, the inner and outer disc scale lengths $\left(h_{\text {in }}, h_{\text {out }}\right)$, etc.

Figure 2 shows the 2D decomposition into a bulge and a broken disc components for IC 1199 as a good example of the typical performance of GASP2D in our sample of galaxies (see also Méndez-Abreu et al. 2017). Top row shows three panels with the observed SDSS $r$-band image (left), the 2D model (middle), and the residuals (model - observed, right). As an average for the entire sample, the residuals oscillate between $\pm 0.45 \mathrm{mag} / \operatorname{arcsec}^{2}$ with the bulk of pixels showing residuals within $\pm 0.15 \mathrm{mag} / \operatorname{arcsec}^{2}(\sim 60 \%)$. The bottom row shows the $\mathrm{SB}$ (left), $e$ (middle), and PA (right) profiles. In those panels, the black dots and shaded areas represent the observed values and errors computed as one sigma of the distribution of values within the ellipse, respectively. The solid light green line is the output from the ellipse IRAF ${ }^{1}$ task applied to the model

1 IRAF is distributed by the National Optical Astronomy Observatory, which is operated by the Association of Universities for Research in Astronomy (AURA) under cooperative agreement with the National Science Foundation. 
image. ellipse fits the galaxy isophotal light distribution by means of ellipses of variable $e$ and PA. The left panel also illustrates the contribution to the light profile coming from the bulge (dashed blue line) and the broken disc (dashed red line) components. Dot-dashed vertical lines and the dot-dashed ellipse in top left panel delimit the inner region (affected by the bulge) and the beginning of the disc-dominated region $\left(R_{\text {lim,in }}\right.$; see Sect. 3.2 for details). Dashed vertical lines and the dashed ellipse in the observed image are located at the break radius $\left(R_{\text {break }}\right)$. Despite the difficulties of fitting complex systems dominated by spiral structures or H II regions with smooth components, the agreement between observed and reconstructed profiles is reasonably good (see bottom row, auxiliary panels).

The results from this analysis are summarised in Table 1 and have been published recently in Méndez-Abreu et al. (2017). Although it is beyond the scope of the present paper, in Appendix A we properly characterise the SB profiles of the galaxies analysed in this work and compare our results with the literature. For the purposes of the main work in this paper, we find that this sample is comprised by 132 type I, 69 type II, and 13 type III galaxies.

\subsection{Colour profiles}

The SDSS data are also used to compute the $g-r, g-i$, and $r-i$ colour profiles for the sample of galaxies by running the ellipse IRAF task to the $g, r$, and $i$ science frames. We fix the $e$ and PA of the successive ellipses matching those of the outer disc (according to the GASP2D analysis in each filter). The onedimensional (1D) light profiles in the three filters are calibrated in flux according to the SDSS DR7 webpage ${ }^{2}$ and subtracted accordingly to obtain the three colour profiles. The errors in the colour profiles are quadratically propagated from the errors in the SB profiles of the bands involved (e.g. $g$ and $r$ bands in the case of the $g-r$ colour) computed as one sigma of the distribution of values (see Sect. 3.1).

We compute linear fits to these colour profiles to quantify and describe their general trends. We restrain the fit to the disc region, avoiding the inner or bulge-dominated area. Considering that the bulge region is where the observed light distribution deviate from the disc exponential profile, we define the inner limit $\left(R_{\text {lim,in }}\right)$ as

$$
\mu_{\text {disc }}(r)-\mu_{\text {obs }}(r)>0.2 \mathrm{mag} / \operatorname{arcsec}^{2} \quad \forall r<R_{\text {lim,in }},
$$

where $r$ is the radius, $\mu_{\text {disc }}$ is the functional shape of the disc light distribution found by GASP2D, and $\mu_{\mathrm{obs}}$ is the observed SB profile (black points in bottom left panel of Fig. 2). The choice of $0.2 \mathrm{mag} / \mathrm{arcsec}^{2}$ in the difference between the theoretical disc and the observed SB profiles allows us to avoid the region dominated by the bulge or the bar in this computation.

For a fair comparison between colour and stellar parameters profile gradients (see Sect. 5), we apply as an outer limit $\left(R_{\text {lim,out }}\right)$ for the colour linear fits that defined from the stellar age and metallicity profiles (see Sect. 4.2). We compute the inner gradients taking into account colour values from $R_{\text {lim,in }}$ to $R_{\text {lim,out }}$ for type I galaxies and from $R_{\mathrm{lim}, \text { in }}$ to $R_{\text {break }}$ for type II and III galaxies. We highlight that we are able to compute high-quality colour profiles beyond $R_{\text {lim,out }}$. However, we fixed the outer limit to $R_{\text {lim,out }}$ to properly compare with the stellar parameters gradients. In Fig. 3 (top panels) we show the colour profiles and results of the linear fits for IC 1199 as an example. The performed linear fits are error weighted and take into account the

\footnotetext{
2 http://www.sdss2.org/dr7/algorithms/fluxcal.html
}
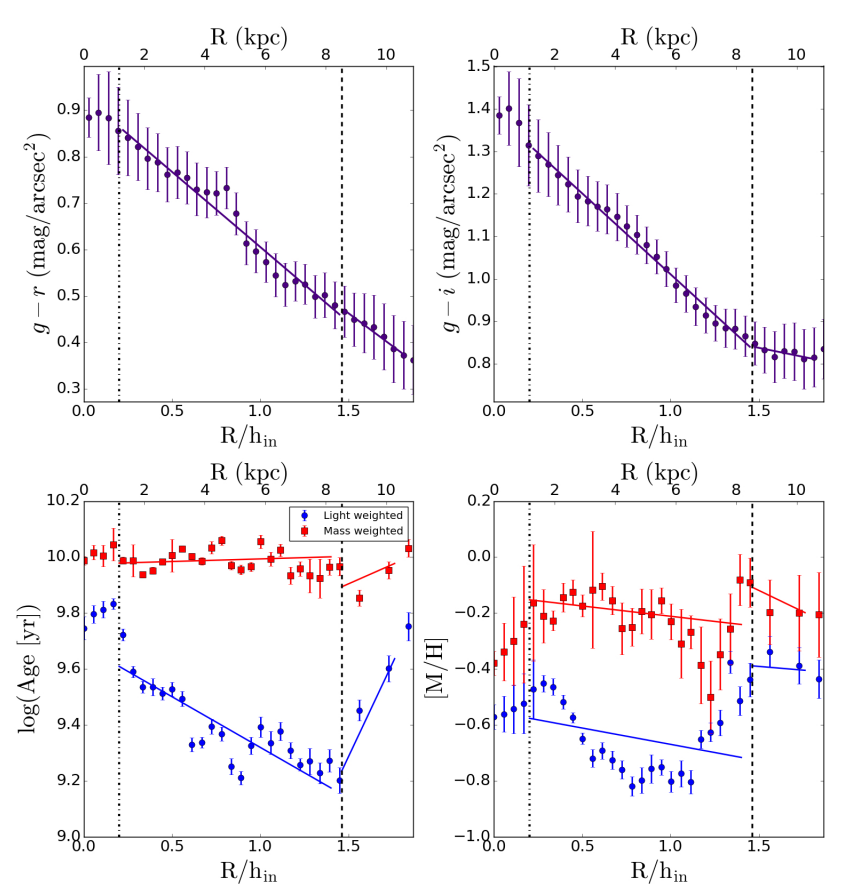

Fig. 3. $g-r$ and $g-i$ colour (top panels) and stellar age and metallicity (bottom panels) profiles for IC 1199 as an example. The colour profiles are represented by means of purple circles (top panels). Blue circles represent light-weighted stellar age or metallicity; red squares symbolise mass-weighted stellar age or metallicity (bottom panels). The dotdashed vertical line delimits the bulge-dominated region $\left(R_{\mathrm{lim}, \text { in }}\right)$, while the dashed vertical is located at the break radius $\left(R_{\text {break }}\right)$.

observational errors of the radial points to derive the parameters of the fit, such as the gradient and its error. The values for all the derived gradients and errors are given in Table B.3.

\section{Spectroscopic analysis}

Our sample of galaxies was drawn from those comprising the CALIFA survey, as already stated in Sect. 2. The CALIFA data (as well as the data from the CALIFA extended projects) were collected at the $3.5 \mathrm{~m}$ telescope at Calar Alto using the PMAS integral field unit spectrograph in its $\mathrm{PPaK}$ configuration (Roth et al. 2005). This project provides high-quality spectra of 667 galaxies in the local Universe $(0.005<z<$ 0.03 , Walcher et al. 2014). Two different observing set-ups were adopted to maximise the scientific impact of the survey, one at high resolution (V1200) and the other at a lower resolution (V500). The wavelength range of the V500 (V1200) data is 3745-7500 $\AA(3650-4840 \AA)$ with a spectral resolution of $F W H M=6.0 \AA(F W H M=2.7 \AA)$. A three-position dithering scheme was chosen to obtain a $100 \%$ coverage of the entire field of view with a final exposure time of $2700 \mathrm{~s}$ for the V500 data (5400 s for the V1200 set-up). We used the CALIFA $\mathrm{COMBO}$ cubes from the version 1.5 of the reduction pipeline (García-Benito et al. 2015). The COMBO cubes are a combination of the V500 and the V1200 (degraded to the V500 resolution) data cubes that avoids vignetting of the data in the blue end of some fibres for the V500 data (see Sánchez et al. 2012). This COMBO dataset displays high-quality spectra across the entire field of view in the wavelength range from $3700 \AA$ to $7500 \AA$.

This dataset has been proven unique to analyse the outer parts of spiral galaxies (see Ruiz-Lara et al. 2016b; Marino et al. 2016; Sánchez-Menguiano et al. 2016b) because of its wide field of view $\left(74^{\prime \prime} \times 64^{\prime \prime}\right)$. In addition, the sky subtraction, which is 
crucial for our purposes, has been thoroughly studied to maximise the information coming from the 36 PMAS sky fibres (see Husemann et al. 2013).

\subsection{Stellar population analysis}

The methodology applied in this work to extract the stellar age and metallicity distributions from the CALIFA data has been extensively tested in previous works (e.g. Sánchez-Blázquez et al. 2011; Seidel et al. 2015). In particular, Ruiz-Lara et al. (2015) apply this method to an integrated spectrum that comes from the scanning a wide region in the bar of the Large Magellanic Cloud to determine its stellar content. We compare those results to the star formation history reconstructed with state-of-the-art methods comparing synthetic and observed colour-magnitude diagrams (Aparicio \& Gallart 2004; Aparicio \& Hidalgo 2009; Hidalgo et al. 2011; Monelli et al. 2010). The good agreement between both approaches encourages us to apply this method to external systems supported by other works in the literature (García-Benito \& Pérez-Montero 2012; Kuncarayakti et al. 2016).

In this section we carefully explain this method. As a preliminary step, we masked the CALIFA data cubes to avoid foreground and background stars and bad or low $\mathrm{S} / \mathrm{N}$ spaxels. This methodology can be divided into three main steps:

i) Stellar kinematics: an adaptive Voronoi method following the Cappellari \& Copin (2003) algorithm is applied to the masked data with a goal continuum $\mathrm{S} / \mathrm{N}$ of 20 and considering just spaxels with a minimum $\mathrm{S} / \mathrm{N}$ of 3 . We apply a stellar kinematics pipeline specifically designed for dealing with the CALIFA data (Falcón-Barroso et al. 2017) to these binned spectra based on the "penalised pixel fitting" code pPXF (Cappellari \& Emsellem 2004; Cappellari et al. 2011). This code uses a maximum-likelihood approach to match the observed spectrum with a combination of stellar templates, once they have been convolved with a line-of-sight velocity distribution (LOSVD). The LOSVD used by pPXF can be described via the Gauss-Hermite parametrisation, allowing the measurement of the velocity, velocity dispersion, and higher order Gauss-Hermite moments up to the h3 and h4 (Gerhard 1993; van der Marel \& Franx 1993). To derive the velocity and velocity dispersion maps we use a subset of the INDOUSv2 library (Valdes et al. 2004). Typical errors in the velocity determination are of the order of 5 to $20 \mathrm{~km} \mathrm{~s}^{-1}$ (for inner to outer spaxels). We use the computed velocity and velocity dispersion maps to shift the observed data cubes to the rest-frame and convolve them to a final FWHM of $8.4 \AA$, resolution that is well suited for analysing low- and intermediate-mass galaxies (Vazdekis et al. 2010). Afterwards, we apply an elliptical integration (annuli) to these corrected data cubes. The width of each annulus is not fixed with the purpose of having spectra with at least a $\mathrm{S} / \mathrm{N}$ of 20 (per $\AA$, in the continuum). The centre, ellipticity, and position angle of the ellipses are fixed, matching the outer disc isophotes from GASP2D (see Sect. 3.1).

ii) Emission line removal: we use GANDALF (Gas AND Absorption Line Fitting; Sarzi et al. 2006; Falcón-Barroso et al. 2006) to decontaminate the CALIFA spectra to have pure absorption spectra. This code is able to simultaneously recover the stellar and ionised gas kinematics and content. GANDALF treats emission lines as additional Gaussian templates to add to the best combination of stellar templates (accounting for the stellar continuum). The code has been modified to take into account the dependency with wavelength of the instrumental FWHM when transformed to velocity units (L. Coccato and M. Sarzi, priv. comm.). In addition, the wavelength range has been limited to the blue part $(3800 \AA$ to $5800 \AA$ ) as most of the spectral features sensitive to the stellar populations are located in this region of the spectrum. We used an optimal subset of the Vazdekis et al. (2010) models (hereafter, V10) with a Kroupa universal initial mass function (Kroupa 2001). These models are based on the MILES library ${ }^{3}$ (Sánchez-Blázquez et al. 2006; Falcón-Barroso et al. 2011) as observed stellar templates. The shape and position of the emission lines computed in this way are subtracted from the observed spectrum (with contributions from stars and gas) to obtain the pureabsorption stellar spectrum.

iii) Stellar content: to recover the stellar content from these "absorption-pure" spectra we use STECKMAP ${ }^{4}$ (STEllar Content and Kinematics via Maximum A Posteriori likelihood; Ocvirk et al. 2006a,b). STECKMAP is aimed at simultaneously recovering the stellar content and stellar kinematics using a Bayesian method via a maximum a posteriori algorithm. It is based on the minimisation of a penalised $\chi^{2}$ while no a priori shape of the solution is assumed (i.e. it is a non-parametric programme). The definition of the penalised $\chi^{2}$ function to minimise is

$Q_{\mu}=\chi^{2}(s(x, Z, g))+P_{\mu}(x, Z, g)$,

where $s$ is the modelled spectrum, which depends on the stellar content (age distribution $x$ and age-metallicity relation, and $Z$ in the above equation) and the stellar kinematics (broadening function, $g$ ). The STECKMAP output consists of three different solutions, the stellar age distribution (SAD), the age-metallicity relation (AMR), and the LOSVD. Those solutions with smooth SAD, AMR, and LOSVD are favoured while solutions with strong variations (i.e. those that are thought to be non-physical) are penalised by means of the penalisation function $\left(P_{\mu}\right.$ in Eq. (2)). This penalisation function is defined as

$P_{\mu}(x, Z, g)=\mu_{x} P(x)+\mu_{Z} P(Z)+\mu_{v} P(g)$.

The different smoothing parameters $\left(\mu_{x}, \mu_{Z}\right.$, and $\left.\mu_{v}\right)$ allow the user to choose the smoothness for the different solutions (SAD, AMR, and LOSVD). The smoothness is completely accomplished by means of the function $P$. This function gives higher penalisation values to strongly oscillated functions while giving low values to smooth solutions. Higher values for the smoothing parameters as input parameters implies that more smoothed solutions are preferred. The function $P$ can also adopt different shapes (for further information see Ocvirk et al. 2006b,a). STECKMAP uses a polynomial to deal with the shape of the continuum, thus, avoiding flux calibration and extinction errors.

Prior to running STECKMAP to the CALIFA data, we investigated which combination of input parameters better fits the CALIFA data. According to those tests, we decided to use a square Laplacian smoothing kernel for the shape of the function $P$ for the SAD and AMR solutions with values $\mu_{x}=0.01$ and $\mu_{Z}=100$, respectively. For further information about these parameters and their selection, see previous works (Ocvirk et al. 2006a,b; Ocvirk 2010; Sánchez-Blázquez et al. 2014b). As we

\footnotetext{
3 The models are publicly available at http://miles.iac.es

4 STECKMAP can be downloaded at http://astro.u-strasbg.fr/ ocvirk/
} 
studied the LOSVD with especially devoted codes (see above), we did not allow STECKMAP to fit the kinematics. We convolved all the observed spectra to a common velocity dispersion of $8.4 \AA$ and shifted them to the rest-frame and we fixed the kinematics while running STECKMAP to these values to reduce the velocity dispersion-metallicity degeneracy reported in Sánchez-Blázquez et al. (2011). In this step we used the entire set of the V10 models with ages ranging from $63 \mathrm{Myr}$ to $17.8 \mathrm{Gyr}$ and metallicities from -2.32 to $+0.2([\mathrm{M} / \mathrm{H}])$ and the same wavelength range that for the emission line removal (3800 $\AA$ to $5800 \AA$ A).

Figure 4 shows an example of a typical, high-quality spectrum analysed with this methodology. The kinematics correction applied to the CALIFA data cubes and the elliptical integration allow us to obtain high-quality spectra even in the outer parts of the analysed galaxies. In this particular case, we show a spectrum (solid black line) at an intermediate-to-large galactocentric distance from IC 1199 ( $\left.24 \operatorname{arcsecs}, 1.34 h_{\text {in }}\right)$. We must note the high quality of the spectrum $(S / N=41.3$ per pixel in the continuum), especially highlighting some features, such as the D4000 break, emission lines ( $\mathrm{H} \beta$, O III, etc.), or absorption features (Ca II, Mg I, etc.). We can observe some remainders of the CALIFA sky subtraction in the O I sky line at $5577 \AA$, which is a region that is masked and thus not considered in the STECKMAP fit. The solid red line shows the STECKMAP fit to the emissionfree spectrum (from GANDALF). The inset is focused on the region where $\mathrm{H} \beta$ and $\mathrm{Mg} \mathrm{I}$ absorption features are located.

\subsection{Age and metallicity radial profiles}

To compute the age and metallicity profiles, both light weighted $(\mathrm{L}-\mathrm{W})$ and mass weighted $(\mathrm{M}-\mathrm{W})$, we average the AMR and the SAD from STECKMAP in logarithmic scale, as extensively done in the literature (e.g. Sánchez-Blázquez et al. 2011, 2014b; Cid Fernandes et al. 2013; González Delgado et al. 2015), i.e.

$$
\begin{aligned}
& \langle\log (\text { Age }[\mathrm{yr}])\rangle_{\mathrm{M}-\mathrm{W}}=\frac{\sum_{i} \operatorname{mass}(i) * \log \left(\mathrm{Age}_{i}\right)}{\sum_{i} \operatorname{mass}(i)} \\
& \langle\log (\operatorname{Age}[\mathrm{yr}])\rangle_{\mathrm{L}-\mathrm{W}}=\frac{\sum_{i} \text { flux }(i) * \log \left(\mathrm{Age}_{i}\right)}{\sum_{i} \operatorname{flux}(i)} \\
& \langle[\mathrm{M} / \mathrm{H}]\rangle_{\mathrm{M}-\mathrm{W}}=\frac{\sum_{i} \operatorname{mass}(i) * \log \left(Z_{i} / Z_{\odot}\right)}{\sum_{i} \operatorname{mass}(i)} \\
& \langle[\mathrm{M} / \mathrm{H}]\rangle_{\mathrm{L}-\mathrm{W}}=\frac{\sum_{i} \text { flux }(i) * \log \left(Z_{i} / Z_{\odot}\right)}{\sum_{i} \text { flux }(i)}
\end{aligned}
$$

where $Z_{\odot}$ is the solar metallicity $(0.02)$ and $\operatorname{mass}(i)$ and flux $(i)$ are the mass and flux of the population with age $=$ Age $_{i}$ and metallicity $=Z_{i}$. Errors in all of the above-defined quantities are computed by means of 25 Monte Carlo simulations, which is a number that has been proven sufficient to provide reasonable errors (Seidel et al. 2015; Ruiz-Lara et al. 2016b). We derive these errors by adding some noise that is consistent with the quality of the original data to the best fit of the observed spectrum and running STECKMAP 25 times to all of these noisy, best-fit spectra. The standard deviation of the 25 recovered age and metallicity values is what we consider the error in each magnitude.

Once we derived the age and metallicity radial distributions, we computed linear fits in a similar way as we explained for the colour profiles (see Sect. 3.2). We used the same definition of $R_{\text {lim,in }}$ as before and the definition of $R_{\text {lim,out }}$ is imposed by the quality of the CALIFA spectroscopic data or performance of the method. Thus, $R_{\text {lim,out }}$ is given by the last radial spectrum

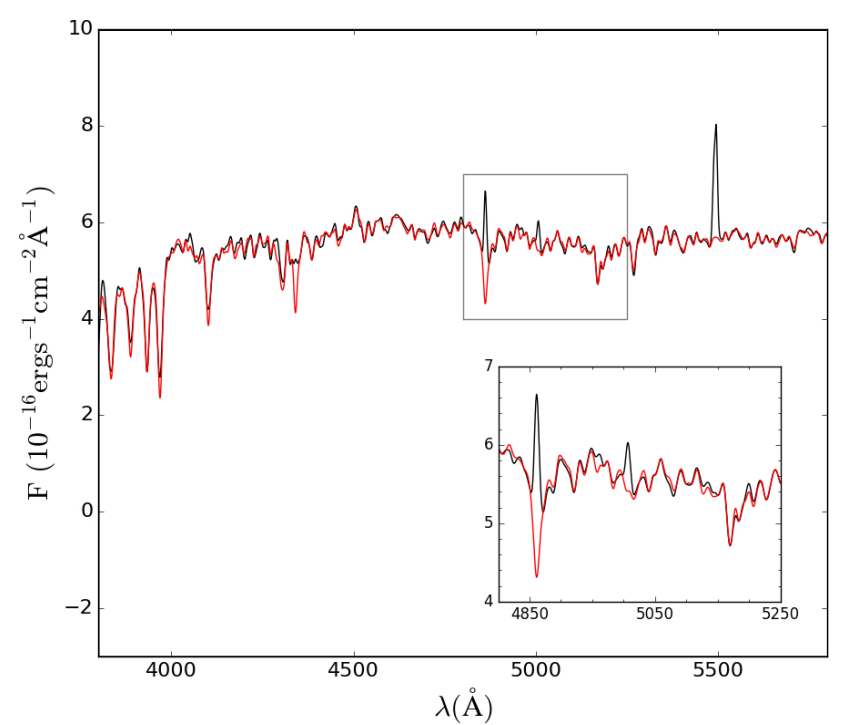

Fig. 4. Example of a typical CALIFA spectrum analysed with the described method. In particular, this is the spectrum of IC 1199 at $24 \operatorname{arcsecs}\left(1.34 h_{\text {in }}\right)$. The solid black line represents the observed, rest-framed spectrum. The solid red line is the STECKMAP best fit to the observed spectrum after removing the gaseous emission lines with GANDALF. The inset is focused on the $\mathrm{H} \beta-\mathrm{Mg} \mathrm{I}(4800-5250 \AA)$. See text for further details.

with a $S / N>20$ or by the last radial spectrum from which reliable stellar population results are drawn. We highlight that in a small number of cases (corresponding to the outermost regions), although the computed $\mathrm{S} / \mathrm{N}$ is higher than 20 , a visual inspection of the GANDALF or STECKMAP fits suggests that they are not good enough (bad emission line removal, deficient continuum shape reconstruction, etc.) and thus, non-reliable stellar population results are obtained. The information from these spectra is discarded. For type I galaxies we computed a single (inner) gradient from $R_{\text {lim,in }}$ to $R_{\text {lim,out }}$ while for type II and III galaxies the outer limit is restricted to the break radius. In this case, again, the performed linear fits are error weighted, taking into account the observational errors of the points to derive the gradient and its error. Figure 3 (bottom panels) shows an example of the typical age and metallicity profiles that we obtained with the CALIFA data. The values of all the gradients and their errors for the age and metallicity profiles (light and mass weighted) are given in Tables B.1 and B.2.

\section{Results}

The procedure explained in the previous sections allows us to characterise the light distribution for the 214 galaxies under analysis and obtain their colour profiles, stellar age, and metallicity profiles. In this paper we focus on the behaviour of such trends in the inner regions to identify differences in their inner gradients for galaxies displaying different SB profiles. The stellar content for some of the galaxies in this sample in the outer regions (i.e. beyond the break radius for type II galaxies or beyond three disc scale lengths for type I galaxies) has been presented in Ruiz-Lara et al. (2016b).

We know from previous works that stellar radial migration exists in spiral galaxies (Feltzing et al. 2001; Nordström et al. 2004; Roškar et al. 2008a; Sánchez-Blázquez et al. 2009; Minchev \& Famaey 2010; Bergemann et al. 2014; Kordopatis et al. 2015; Sánchez-Menguiano et al. 2016a). Moreover, if 

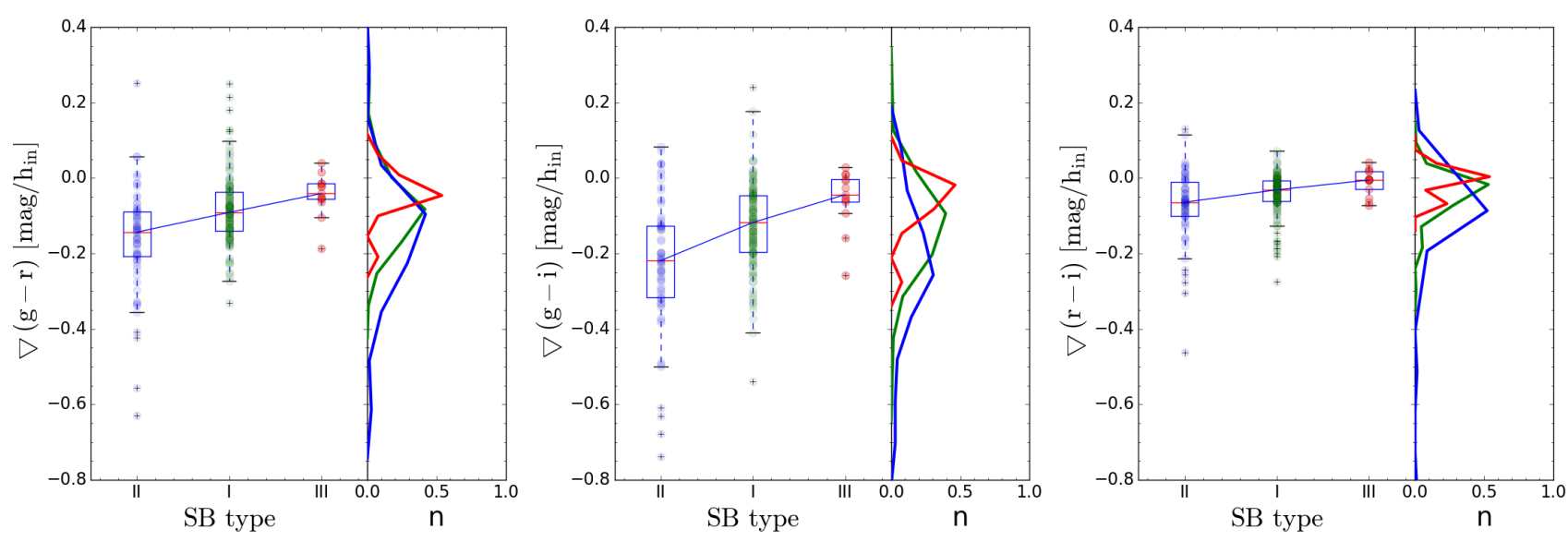

Fig. 5. Distribution of the inner $g-r(l e f t), g-i$ (middle), and $r-i$ (right) colour gradients as a function of the surface brightness profiles of the galaxies represented as box plots. The boxes extend from the lower to upper quartile values of the gradient distributions with a red line at the median. The whiskers extend from the box to show the range of the data. Outlier points are those past the end of the whiskers and are represented with crosses. We also show the histograms for each distribution colour coded according to the SB type: type I (green), type II (blue), and type III (red). Dots with transparency represent all the observational values. The median values of all the distributions are linked using a blue line to highlight the tendency.

radial redistribution of material is important, it must have an effect in the stellar content of the inner and outer regions. As a consequence, if there is a relation between radial migration and SB profiles, galaxies displaying different SB profiles should display differences in their inner stellar content. Therefore, the analysis of the inner colour profiles (Sect. 5.1) along with the inner stellar age and metallicity gradients (Sect. 5.2) segregating the different SB profile types, can help shed light on the role of radial migration in the shaping of stellar parameter and light distribution profiles.

\subsection{Inner colour gradients}

The analysis of the light distribution carried out along this work (see Sect. 3) allows us to obtain colour radial profiles. Although we know that drawing stellar population information from optical colours might not be an optimal approach (Ganda et al. 2009), for the sake of completeness we studied the colour inner gradient distribution for type I, II, and III galaxies.

Figure 5 shows the distribution of the inner gradients for the $(g-r),(g-i)$, and $(r-i)$ colour profiles as box plots (main plots) and by means of histograms (right-hand auxiliary panels). The error-weighted mean values of the inner gradient distribution for the $(g-r),(g-i)$ and $(r-i)$ colour profiles are shown in Table 2. We can see that gradient distributions for type I, II, and III galaxies are different. Type II galaxies present the steepest gradients of all, followed by type I and type III systems (displaying the shallowest profiles). To further quantify such a result, we make use of two different statistical tests, the Kolmogorov-Smirnov (KS) and Anderson-Darling (AD) tests, to check whether or not these distributions are drawn from the same distribution. These tests are used to compare two samples and provide estimations on how different these two distributions are by means of a parameter called " $p$-value". If this statistical parameter is below a significance level (generally $\sim 0.05$ ) then, we can reject the "null hypothesis", i.e. we can conclude that the two samples are drawn from different distributions. The corresponding " $p$-values" for all these tests can be found in Table 3. Very low $p$-values are found using the KS tests when comparing the colour inner gradient distributions for types I and II galaxies as well as those of types I and III and types II and III. Although the size of the sample under analysis in this work is large enough to be analysed with a KS test that is suited for large samples, we decided to use the AD test too, which is better suited for small samples. The $p$-values obtained using this second statistical test (AD) agree with those found with a KS test, with $p$-values always well below the significance level. Then, we can conclude that the colour profile inner gradient distributions for type I, II, and III galaxies seem to be drawn from different distributions, in which type II galaxies display steeper negative trends than type I and III systems with the latter showing the shallowest profiles.

\subsection{Inner stellar parameters gradients}

To further test our hypothesis of radial migration, which affects the inner stellar content of galaxies with different SB profiles differently, Fig. 6 shows the distribution of the inner gradients of the $\log ($ age $[\mathrm{yr}])$ and $[\mathrm{M} / \mathrm{H}]$ profiles $(\mathrm{L}-\mathrm{W}$ and $\mathrm{M}-\mathrm{W})$ for galaxies with different SB profiles in a similar way as presented in Fig. 5 for the colour gradients. After a visual inspection, we can claim that similar trends to those shown by the colour profiles are found especially in the case of L-W quantities, although in this case those trends are not as clear as in the previous case. The observed tendency suggests that as we move from type II galaxies to type I and type III systems the slope of the L-W profiles becomes shallower. The trend is also observed but to a lesser degree in the case of the M-W quantities. The actual values for the error-weighted average gradients and their dispersions for each SB profile type are shown in Table 4.

The average values confirm the first visual impression: type II galaxies present the steepest profiles and type III the shallowest with type I spirals displaying an intermediate behaviour in L-W parameters. However, although a similar trend is found for the gradients from the M-W quantities, we find that M-W age gradients, where type I and III galaxies display similar average values, are the exception. As in the case of the colour gradients, and to further quantify such a result, we make use of the KS and AD statistical tests. The " $p$-values" for all these tests can be found in Table 5. The KS tests seem to indicate that the observed distributions for type I and III galaxies are basically identical in the case of the M-W quantities with high $p$-values $(0.62$ and 0.37 , respectively). However, slight differences are found in the case of the L-W age gradients ( $p$-value of 0.07 , around the 
T. Ruiz-Lara et al.: Observational hints of radial migration in disc galaxies from CALIFA

Table 2. Error-weighted average, its error and dispersion (the later in parenthesis) values of the inner gradients for the $g-r, g-i$, and $r-i$ colour profiles (see text for details).

\begin{tabular}{cccc}
\hline \hline & & SB profile & \\
& Type II & Type I & Type III \\
\hline$g-r$ & $-0.12 \pm 0.02(0.16)$ & $-0.084 \pm 0.007(0.08)$ & $-0.04 \pm 0.02(0.07)$ \\
$g-i$ & $-0.17 \pm 0.03(0.20)$ & $-0.123 \pm 0.010(0.12)$ & $-0.05 \pm 0.02(0.08)$ \\
$r-i$ & $-0.05 \pm 0.01(0.13)$ & $-0.041 \pm 0.005(0.07)$ & $-0.01 \pm 0.01(0.04)$ \\
\hline
\end{tabular}

Notes. Units are in $\mathrm{mag} / h_{\mathrm{in}}$.

Table 3. Statistical " $p$-values" for the KS (AD tests in parenthesis) when comparing the inner gradients distributions for type I vs. type II, type I vs. type III, and type II vs. type III galaxies of the colour profiles analysed in this work $(g-r, g-i$, and $r-i)$.

\begin{tabular}{lccc}
\hline \hline SB profiles & $(g-r)$ & $(g-i)$ & $(r-i)$ \\
\hline I vs. II & $0.001(0.0001)$ & $0.0001(0.00002)$ & $0.0001(0.0005)$ \\
I vs. III & $0.00001(0.012)$ & $0.0(0.01)$ & $0.0(0.04)$ \\
II vs. III & $0.0(0.0003)$ & $0.0(0.0004)$ & $0.0(0.007)$ \\
\hline
\end{tabular}

Table 4. Error-weighted average, its error and dispersion (the later in parenthesis) values of the inner gradients for the stellar age and metallicity profiles (see text for details).

\begin{tabular}{lccc}
\hline \hline & & SB profile & \\
& Type II & Type I & Type III \\
\hline L-W age & $-0.30 \pm 0.06(0.51)$ & $-0.18 \pm 0.02(0.26)$ & $-0.09 \pm 0.03(0.11)$ \\
L-W metallicity & $-0.15 \pm 0.06(0.47)$ & $-0.09 \pm 0.02(0.18)$ & $-0.04 \pm 0.02(0.06)$ \\
M-W age & $-0.06 \pm 0.02(0.14)$ & $-0.03 \pm 0.01(0.13)$ & $-0.03 \pm 0.02(0.06)$ \\
M-W metallicity & $-0.14 \pm 0.03(0.26)$ & $-0.09 \pm 0.02(0.26)$ & $-0.06 \pm 0.02(0.08)$ \\
\hline
\end{tabular}

Notes. L-W stands for light-weighted quantities whereas M-W stands for mass weighted. Units are in dex $/ h_{\text {in }}$.

significance level) and clear differences in the case of the L-W metallicity gradients ( $p$-value of 0.04 , below the significance level). Similar results arise from the AD tests, i.e. the samples for type I and III galaxies follow the same distribution in the case of M-W quantities but differences seem to appear when we compare the distributions of the L-W gradients. Regarding the distributions shown by type II galaxies, KS and AD tests suggest that they are drawn from different distributions than type I and III systems if we pay attention to both $\mathrm{L}-\mathrm{W}$ and $\mathrm{M}-\mathrm{W}$ profiles. However, this statement should be taken with caution because in the case of the metallicity gradients the $p$-values are in some cases similar to, or even slightly above, the significance level and thus the observed differences, if any, are almost negligible.

We also reproduced the analysis shown in Fig. 6, but we distinguished between barred and unbarred systems instead of SB profile types to assess the effect of the presence of a bar in shaping the stellar parameter profiles. The distributions found for barred and unbarred galaxies are completely consistent, thus suggesting that presence of bars does not seem to have a strong effect in shaping the stellar parameter profiles (in agreement with the results found in Sánchez-Blázquez et al. 2014b; Marino et al. 2016).

The similar behaviours of the inner gradients of the stellar parameter and colour profiles for type I, II, and III galaxies, especially in the case of the L-W quantities, can be interpreted as a reinforcement of the results outlined above. In addition, this similarity might shed some light into whether optical colours can be used as a proxy for stellar ages or metallicities when looking for statistical trends in large samples of galaxies. However, to make this claim further investigations and comparisons between colour and stellar parameter profiles are needed.

It is worth mentioning the fact that, apart from the abovementioned differences, the distributions of inner gradients (colours and stellar parameters) also present different dispersions (see parenthesis in Tables 2 and 4). The values of the inner gradients for type III galaxies always present lower dispersions than type I or II systems. However, this is understandable considering the low number of galaxies exhibiting a type III profile (13). It is more intriguing that even though the number of type I galaxies is much larger than the number of type II galaxies (132 versus 69), type II galaxies present larger dispersion for all analysed quantities. Whether this is a consequence of the different possible processes shaping the type II SB profiles or a direct consequence of the lower degree of mixing induces by radial migration needs to be further investigated.

\section{Discussion}

Our hypothesis states that $\mathrm{i}$ ) if migration is capable of redistributing a sufficient mass fraction as to affect the observed inner stellar content and ii) different radial migration efficiencies affect the shaping of the outer light distributions, galaxies with different SB profiles should display differences in their inner colour and stellar age and metallicity gradients. The results presented in this paper suggest that this can be the case: type II galaxies might present the lowest degree of radial migration, thereby causing the stellar population gradients to be steeper, while type III systems might display a larger degree of mixing, thus resulting in shallower gradients. The fact that type I systems tend to display average values in between type II and III systems and slightly different distributions to both types further supports this scenario. In this work, we have also checked if the differences in the stellar parameter gradients for type II galaxies (steep gradients) are due to a more efficient current star formation in these systems. The analysis of the light fraction of stars younger than $150 \mathrm{Myr}$ yields no differences between type I, II, and III galaxies discarding a 

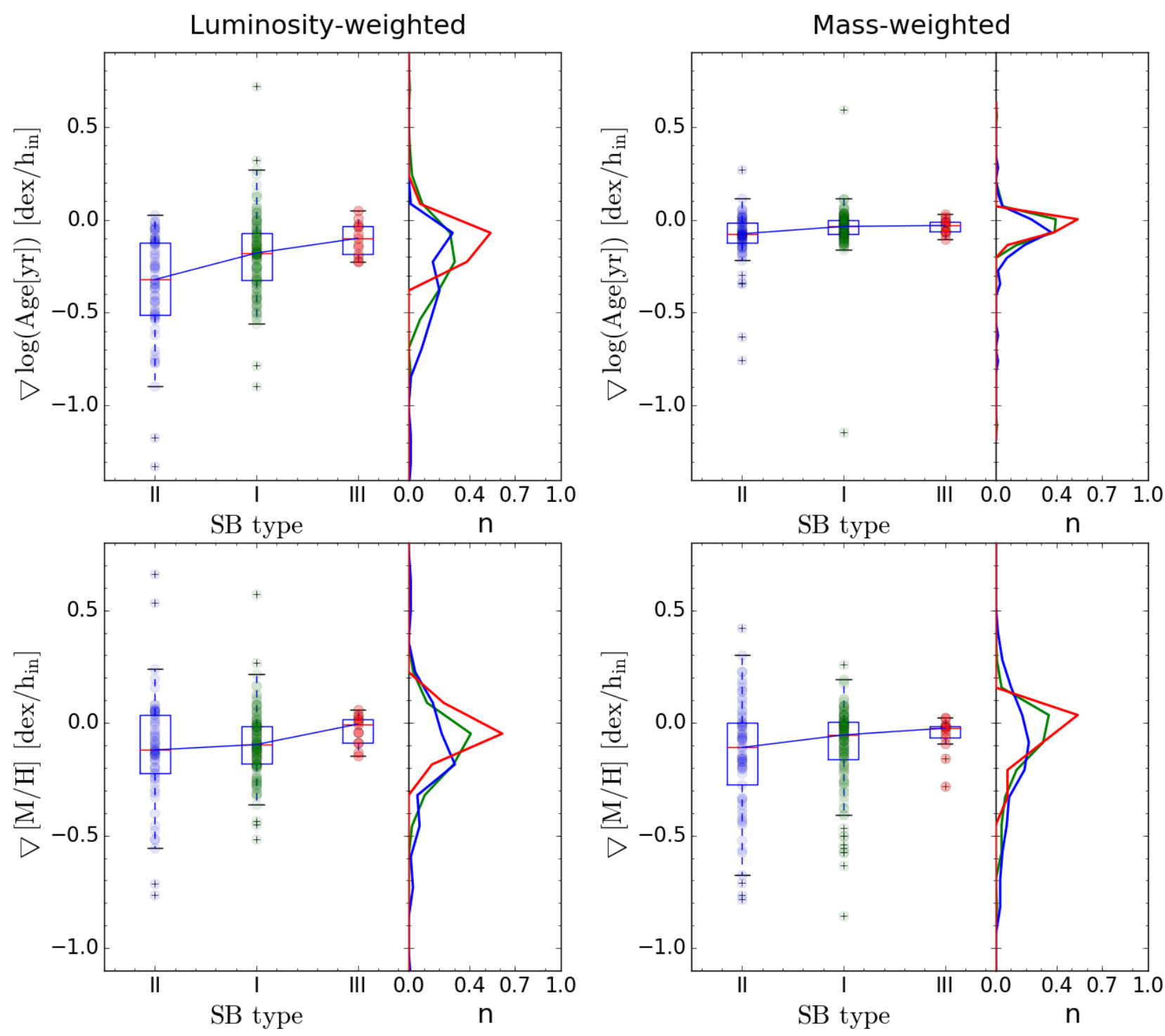

Fig. 6. Distribution of the inner gradients of the $\log (\operatorname{age}[\mathrm{yr}])$ and $[\mathrm{M} / \mathrm{H}]$ profiles as a function of the surface brightness profiles of the galaxies represented as box plots. The boxes extend from the lower to upper quartile values of the gradient distributions with a red line at the median. The whiskers extend from the box to show the range of the data. Outlier points are those past the end of the whiskers and are represented with crosses. We also show the histograms for each distribution colour coded according to the SB type: type I (green), type II (blue), and type III (red). Top left: luminosity-weighted age is shown. Top right: mass-weighted age is shown. Bottom left: luminosity-weighted [M/H] is shown. Bottom right: mass-weighted $[\mathrm{M} / \mathrm{H}]$ is shown. Dots with transparency represent all the observational values. The median values of all the distributions are linked using a blue line to highlight the tendency.

Table 5. Statistical " $p$-values" for the KS (AD tests in parenthesis) when comparing the inner gradient distributions for type I vs. type II, type I vs. type III and type II vs. type III galaxies of the stellar properties analysed in this work (L-W and M-W age as well as L-W and M-W metallicity).

\begin{tabular}{lcccc}
\hline \hline SB profiles & L-W age & M-W age & L-W $[M / H]$ & M-W [M/H] \\
\hline I vs. II & $0.005(0.0003)$ & $0.001(0.004)$ & $0.07(0.05)$ & $0.07(0.06)$ \\
I vs. III & $0.07(0.07)$ & $0.62(0.60)$ & $0.04(0.02)$ & $0.37(0.23)$ \\
II vs. III & $0.001(0.001)$ & $0.02(0.03)$ & $0.03(0.03)$ & $0.08(0.05)$ \\
\hline
\end{tabular}

recent star formation burst as the cause of the steeper L-W age profiles in type II systems. Although we are tempted to claim that these results are evidence of different radial migration efficiencies for galaxies displaying different SB profiles, we must bear in mind that other processes involved in the formation of spiral galaxies besides migration can be also at play (as suggested in Ruiz-Lara et al. 2016b).

Other theoretical and observational works have also tried to understand the role of radial migration on shaping light distribution and the stellar content in spiral galaxies. Roškar et al. (2008b) used their $\mathrm{SPH}+N$-body simulations to propose that SB and age profiles were intimately related to radial migration, i.e. radial migration, along with a radial cut-off of the star formation, was the cause of downbending of the light profile and ageing of the outer disc. In Ruiz-Lara et al. (2016a), the authors have analysed the origin of the outer ageing in a set of simulated spiral galaxies (RaDES, Few et al. 2012) and have found that type I, II, and III galaxies show "U-shape" age 
profiles even when radial migration is not considered. Consistent with that work, Ruiz-Lara et al. (2016b) analysed spectroscopic data from the CALIFA survey to obtain "U-shape" age profiles indistinctly for type I and II galaxies suggesting that the shaping of the SB and the outer ageing on the stellar age profiles are unconnected. In addition, recent observational works suggest the existence of old outer discs formed through radial migration and/or satellite accretion in a wide variety of galactic systems. Zhang et al. (2012) have analysed broadband images of 34 dwarf irregular galaxies and have found some hints of old stellar populations over the entire disc from their spectral energy distribution (SED) modelling. Similar results have been found by Zheng et al. (2015), who analysed SED modelling of 698 spiral galaxies from the Pan-STARRS1 Medium Deep Survey images. Dale et al. (2016) have analysed deep optical and near-infrared images of a sample of 15 nearby spiral galaxies and have found results that are consistent with an inside-out disc formation coupled with an old stellar outer disc. Analyses of deep colour magnitude diagrams have also claimed the presence of old stars in the outskirts of very nearby systems (Bernard et al. 2012, 2015; Radburn-Smith et al. 2012).

The variety of results found in the previous mentioned works suggest that we are still far from understanding the real role of radial migration in shaping the stellar distribution (light, age, and metallicity) of spiral galaxies. Although it seems that radial migration is present in galaxies, we still cannot decouple the possible effects of recent star formation, satellite accretion, or radial migration in modelling observed galaxies. The observed properties can be the consequence of a complex process, involving not only radial migration but multicomponent star formation recipes (Elmegreen \& Hunter 2006), combinations of star formation thresholds, and collapse of the protogalactic cloud conserving angular momentum (van den Bosch 2001), satellite accretion, etc.

However, the results shown in this paper are in agreement with the speculations presented in Sánchez-Blázquez et al. (2009) and might indicate that there can be a radial migration efficiency transition between type II, I, and III. If this is the case, the factor that determines the type of SB profile to exhibit would be the amount of outwards migrated stars. If outwards radial migration is high enough, this migration could change the observed SB profiles of spiral galaxies from type II to I or even III (with the combined action of satellite accretion).

This work helps us to constrain future theoretical works through studying the origin of the outskirts of spiral galaxies and proposing a possible scenario to explain the different observed SB profiles. Although we cannot conclude whether radial migration is the main mechanism or it is just one of many mechanisms shaping the different SB profiles, we can claim that the amount of stars that are currently displaced from their birth locations can represent a significant fraction of the total stellar mass as to affect the inner stellar age and metallicity profiles.

\section{Conclusions}

In this work we present stellar age and metallicity profiles from full-spectrum fitting analysis of the CALIFA IFS data of a sample of 214 spiral galaxies. We have carefully analysed their 2D light distribution to characterise their SB profiles and obtain colour profiles from the analysis of SDSS data. Our main conclusions are as follows:

i) There are statistical differences in the behaviour of the colour profiles $(g-r, g-i$ and $r-i)$ in the inner parts for type II galaxies compared to type I and III systems. Type II galaxies tend to display the steepest profiles and type III the shallowest while type I galaxies show an intermediate behaviour.

ii) Similar findings are obtained when computing the inner gradients of the L-W stellar age and metallicity trends in the inner regions. The trend is also reproduced in the case of M-W quantities but to a lesser extent.

iii) Conclusions i) and ii) together suggest a segregation in the radial migration efficiency for type I, II, and III galaxies, where type III systems are the most efficient (shallowest profiles) and type II are the least efficient systems (steepest profiles). These findings allow us to suggest that radial migration seems to have an effect in shaping the stellar content in spiral galaxies (light, stellar age, and stellar metallicity distributions). The efficiency of the outwards radial migration might be the factor that determines the observed SB profile in a galaxy, from type II (low efficiency) to type III (high efficiency). However, a joint effort of simulations and observations is needed in order to properly assess a) the actual role of radial migration in galaxy evolution and its causes; b) the role of satellite accretion; and c) whether these different behaviours between type I, II, and III systems are imprinted at birth or actually acquired via radial migration.

Acknowledgements. We thank the referee for very useful suggestions and comments that have helped improve the current version of this manuscript. The authors also thank L. Coccato and M. Sarzi for the new version of the GANDALF code taking into account the dependency with wavelength of the instrumental FWHM. We would like to thank Reynier Peletier and Stéphane Courteau for very helpful suggestions and discussions that have considerably improved this paper. This paper is (partially) based on data obtained by the CALIFA Survey, funded by the Spanish Ministery of Science under grant ICTS-2009-10, and the Centro Astronómico Hispano-Alemán. T.R.L. thanks the support of the Spanish Ministerio de Educación, Cultura y Deporte by means of the FPU fellowship. This research has been supported by the Spanish Ministry of Science and Innovation (MICINN) under grants AYA2014-53506-P, AYA2007-67625-C02-02, AYA2014-56795-P and Consolider-Ingenio CSD2010-00064; and by the Junta de Andalucía (FQM-108). L.G. was supported in part by the US National Science Foundation under Grant AST-1311862. I.M. would like to thank support under grants AYA2013-42227-P and AYA2016-76682-C3-1-P. S.Z. has been supported by the EU Marie Curie Career Integration Grant SteMaGE No. PCIG12-GA2012-326466 (Call Identifier: FP7-PEOPLE-2012 CIG). J.F.B. thanks the support received under grant AYA2016-77237-C3-1-P. A.d.L.C. acknowledges support from the CONACyT-125180, DGAPA-IA100815 and DGAPA-IA101217 projects. R.A.M. acknowledges support by the Swiss National Science Foundation. J.M.A. thanks support from the support from the European Research Council Starting Grant (SEDmorph; P.I. V. Wild) and MINECO through the grant AYA2013-43188-P. Funding for the creation and distribution of the SDSS Archive has been provided by the Alfred P. Sloan Foundation, the Participating Institutions, the National Aeronautics and Space Administration, the National Science Foundation, the US Department of Energy, the Japanese Monbukagakusho, and the Max Planck Society. The SDSS Web site is http://www . sdss.org/. The SDSS is managed by the Astrophysical Research Consortium (ARC) for the Participating Institutions. The Participating Institutions are The University of Chicago, Fermilab, the Institute for Advanced Study, the Japan Participation Group, The Johns Hopkins University, the Korean Scientist Group, Los Alamos National Laboratory, the Max-Planck-Institute for Astronomy (MPIA), the Max-Planck-Institute for Astrophysics (MPA), New Mexico State University, University of Pittsburgh, University of Portsmouth, Princeton University, the United States Naval Observatory, and the University of Washington.

\section{References}

Abazajian, K. N., Adelman-McCarthy, J. K., Agüeros, M. A., et al. 2009, ApJS, 182,543

Aparicio, A., \& Gallart, C. 2004, AJ, 128, 1465

Aparicio, A., \& Hidalgo, S. L. 2009, AJ, 138, 558

Beasley, M. A., San Roman, I., Gallart, C., Sarajedini, A., \& Aparicio, A. 2015, MNRAS, 451, 3400

Bell, E. F., \& de Jong, R. S. 2000, MNRAS, 312, 497

Bergemann, M., Ruchti, G. R., Serenelli, A., et al. 2014, A\&A, 565, A89

Bernard, E. J., Ferguson, A. M. N., Barker, M. K., et al. 2012, MNRAS, 420, 2625 
Bernard, E. J., Ferguson, A. M. N., Richardson, J. C., et al. 2015, MNRAS, 446, 2789

Bica, E. 1988, A\&A, 195, 76

Bica, E., \& Alloin, D. 1986a, A\&A, 162, 21

Bica, E., \& Alloin, D. 1986b, A\&AS, 66, 171

Bica, E., Alloin, D., \& Schmitt, H. R. 1994, A\&A, 283, 805

Bird, J. C., Kazantzidis, S., \& Weinberg, D. H. 2012, MNRAS, 420, 913

Bland-Hawthorn, J., Vlajić, M., Freeman, K. C., \& Draine, B. T. 2005, ApJ, 629 239

Cappellari, M., \& Copin, Y. 2003, MNRAS, 342, 345

Cappellari, M., \& Emsellem, E. 2004, PASP, 116, 138

Cappellari, M., Emsellem, E., Krajnović, D., et al. 2011, MNRAS, 413, 813

Cid Fernandes, R., Mateus, A., Sodré, L., Stasińska, G., \& Gomes, J. M. 2005 , MNRAS, 358, 363

Cid Fernandes, R., Pérez, E., García Benito, R., et al. 2013, A\&A, 557, A86

Cole, A. A., Skillman, E. D., Tolstoy, E., et al. 2007, ApJ, 659, L17

Dale, D. A., Beltz-Mohrmann, G. D., Egan, A. A., et al. 2016, AJ, 151, 4

de Jong, R. S. 1996, A\&A, 313, 377

Debattista, V. P., Mayer, L., Carollo, C. M., et al. 2006, ApJ, 645, 209

Elmegreen, B. G., \& Hunter, D. A. 2006, ApJ, 636, 712

Erwin, P., Pohlen, M., \& Beckman, J. E. 2008, AJ, 135, 20

Faber, S. M., Friel, E. D., Burstein, D., \& Gaskell, C. M. 1985, ApJS, 57, 711

Falcón-Barroso, J., Bacon, R., Bureau, M., et al. 2006, MNRAS, 369, 529

Falcón-Barroso, J., Sánchez-Blázquez, P., Vazdekis, A., et al. 2011, A\&A, 532, A95

Falcón-Barroso, J., Lyubenova, M., van de Ven, G., et al. 2017, A\&A, 597, A48

Feltzing, S., Holmberg, J., \& Hurley, J. R. 2001, A\&A, 377, 911

Few, C. G., Gibson, B. K., Courty, S., et al. 2012, A\&A, 547, A63

Freeman, K. C. 1970, ApJ, 160, 811

Gallart, C., Monelli, M., Mayer, L., et al. 2015, ApJ, 811, L18

Gallazzi, A., Charlot, S., Brinchmann, J., White, S. D. M., \& Tremonti, C. A. 2005, MNRAS, 362, 41

Gallazzi, A., Charlot, S., Brinchmann, J., \& White, S. D. M. 2006, MNRAS, 370,1106

Gallazzi, A., Brinchmann, J., Charlot, S., \& White, S. D. M. 2008, MNRAS, 383,1439

Ganda, K., Peletier, R. F., Balcells, M., \& Falcón-Barroso, J. 2009, MNRAS, 395,1669

García-Benito, R., \& Pérez-Montero, E. 2012, MNRAS, 423, 406

García-Benito, R., Zibetti, S., Sánchez, S. F., et al. 2015, A\&A, 576, A135

Gerhard, O. E. 1993, MNRAS, 265, 213

González Delgado, R. M., García-Benito, R., Pérez, E., et al. 2015, A\&A, 581, A103

González Delgado, R. M., Cid Fernandes, R., Pérez, E., et al. 2016, A\&A, 590, A44

Gorgas, J., Faber, S. M., Burstein, D., et al. 1993, ApJS, 86, 153

Gutiérrez, L., Erwin, P., Aladro, R., \& Beckman, J. E. 2011, AJ, 142, 145

Heavens, A. F., Jimenez, R., \& Lahav, O. 2000, MNRAS, 317, 965

Hidalgo, S. L., Aparicio, A., Skillman, E., et al. 2011, ApJ, 730, 14

Husemann, B., Jahnke, K., Sánchez, S. F., et al. 2013, A\&A, 549, A87

Jansen, R. A., Fabricant, D., Franx, M., \& Caldwell, N. 2000, ApJS, 126, 331

Kauffmann, G., Heckman, T. M., White, S. D. M., et al. 2003, MNRAS, 341, 33

Koleva, M., Prugniel, P., Bouchard, A., \& Wu, Y. 2009, A\&A, 501, 1269

Kordopatis, G., Gilmore, G., Steinmetz, M., et al. 2013, AJ, 146, 134

Kordopatis, G., Binney, J., Gilmore, G., et al. 2015, MNRAS, 447, 3526

Kroupa, P. 2001, MNRAS, 322, 231

Kuncarayakti, H., Galbany, L., Anderson, J. P., Krühler, T., \& Hamuy, M. 2016 A\&A, 593, A78

Kuntschner, H., Emsellem, E., Bacon, R., et al. 2010, MNRAS, 408, 97

MacArthur, L. A., Courteau, S., Bell, E., \& Holtzman, J. A. 2004, ApJS, 152, 175

MacArthur, L. A., González, J. J., \& Courteau, S. 2009, MNRAS, 395, 28

Marino, R. A., Gil de Paz, A., Sánchez, S. F., et al. 2016, A\&A, 585, A47

Markwardt, C. B. 2009, in Astronomical Data Analysis Software and Systems XVIII, eds. D. A. Bohlender, D. Durand, \& P. Dowler, ASP Conf. Ser., 411, 251

Martínez-Serrano, F. J., Serna, A., Doménech-Moral, M., \& DomínguezTenreiro, R. 2009, ApJ, 705, L133

Méndez-Abreu, J., Aguerri, J. A. L., Corsini, E. M., \& Simonneau, E. 2008, A\&A, 478, 353

Méndez-Abreu, J., Debattista, V. P., Corsini, E. M., \& Aguerri, J. A. L. 2014, A\&A, 572, A25

Méndez-Abreu, J., Ruiz-Lara, T., Sánchez-Menguiano, L., et al. 2017, A\&A, 598, A32

Minchev, I., \& Famaey, B. 2010, ApJ, 722, 112

Minchev, I., Famaey, B., Combes, F., et al. 2011, A\&A, 527, A147
Minchev, I., Famaey, B., Quillen, A. C., et al. 2012a, A\&A, 548, A127 Minchev, I., Famaey, B., Quillen, A. C., et al. 2012b, A\&A, 548, A126 Monelli, M., Hidalgo, S. L., Stetson, P. B., et al. 2010, ApJ, 720, 1225 Moré, J. J., Garbow, B. S., \& Hillstrom, K. E. 1980, Argonne National Laboratory Report ANL-80-74

Morelli, L., Corsini, E. M., Pizzella, A., et al. 2015, MNRAS, 452, 1128 Morelli, L., Parmiggiani, M., Corsini, E. M., et al. 2016, MNRAS, 463, 4396 Muñoz-Mateos, J. C., Gil de Paz, A., Zamorano, J., et al. 2009, ApJ, 703, 1569 Nordström, B., Mayor, M., Andersen, J., et al. 2004, A\&A, 418, 989 Ocvirk, P. 2010, ApJ, 709, 88

Ocvirk, P., Pichon, C., Lançon, A., \& Thiébaut, E. 2006a, MNRAS, 365, 74 Ocvirk, P., Pichon, C., Lançon, A., \& Thiébaut, E. 2006b, MNRAS, 365, 46 Peletier, R. F. 1993, A\&A, 271, 51

Peletier, R. F., \& Balcells, M. 1996, AJ, 111, 2238

Peletier, R. F., Falcón-Barroso, J., Bacon, R., et al. 2007, MNRAS, 379, 445

Pérez, I., \& Sánchez-Blázquez, P. 2011, A\&A, 529, A64

Pérez, E., Cid Fernandes, R., González Delgado, R. M., et al. 2013, ApJ, 764, L1 Pohlen, M., \& Trujillo, I. 2006, A\&A, 454, 759

Radburn-Smith, D. J., Roškar, R., Debattista, V. P., et al. 2012, ApJ, 753, 138

Reichardt, C., Jimenez, R., \& Heavens, A. F. 2001, MNRAS, 327, 849

Roediger, J. C., Courteau, S., MacArthur, L. A., \& McDonald, M. 2011a, MNRAS, 416, 1996

Roediger, J. C., Courteau, S., McDonald, M., \& MacArthur, L. A. 2011b, MNRAS, 416, 1983

Roediger, J. C., Courteau, S., Sánchez-Blázquez, P., \& McDonald, M. 2012, ApJ, 758,41

Rose, J. A. 1984, AJ, 89, 1238

Roth, M. M., Kelz, A., Fechner, T., et al. 2005, PASP, 117, 620

Roškar, R., Debattista, V. P., Quinn, T. R., Stinson, G. S., \& Wadsley, J. 2008a, ApJ, 684, L79

Roškar, R., Debattista, V. P., Stinson, G. S., et al. 2008b, ApJ, 675, L65

Roškar, R., Debattista, V. P., Quinn, T. R., \& Wadsley, J. 2012, MNRAS, 426, 2089

Ruiz-Lara, T., Pérez, I., Gallart, C., et al. 2015, A\&A, 583, A60

Ruiz-Lara, T., Few, C. G., Gibson, B. K., et al. 2016a, A\&A, 586, A112

Ruiz-Lara, T., Pérez, I., Florido, E., et al. 2016b, MNRAS, 456, L35

Sánchez, S. F., Rosales-Ortega, F. F., Kennicutt, R. C., et al. 2011, MNRAS, 410 , 313

Sánchez, S. F., Kennicutt, R. C., Gil de Paz, A., et al. 2012, A\&A, 538, A8

Sánchez, S. F., García-Benito, R., Zibetti, S., et al. 2016, A\&A, 594, A36

Sánchez-Blázquez, P., Peletier, R. F., Jiménez-Vicente, J., et al. 2006, MNRAS 371,703

Sánchez-Blázquez, P., Courty, S., Gibson, B. K., \& Brook, C. B. 2009, MNRAS, 398, 591

Sánchez-Blázquez, P., Ocvirk, P., Gibson, B. K., Pérez, I., \& Peletier, R. F. 2011, MNRAS, 415, 709

Sánchez-Blázquez, P., Rosales-Ortega, F., Diaz, A., \& Sánchez, S. F. 2014a, MNRAS, 437, 1534

Sánchez-Blázquez, P., Rosales-Ortega, F. F., Méndez-Abreu, J., et al. 2014b, A\&A, 570, A6

Sánchez-Menguiano, L., Sánchez, S. F., Kawata, D., et al. 2016a, ApJ, 830, L40

Sánchez-Menguiano, L., Sánchez, S. F., Pérez, I., et al. 2016b, A\&A, 587, A70

Sarzi, M., Falcón-Barroso, J., Davies, R. L., et al. 2006, MNRAS, 366, 1151

Seidel, M. K., Cacho, R., Ruiz-Lara, T., et al. 2015, MNRAS, 446, 2837

Sellwood, J. A., \& Binney, J. J. 2002, MNRAS, 336, 785

Steinmetz, M., Zwitter, T., Siebert, A., et al. 2006, AJ, 132, 1645

Tojeiro, R., Heavens, A. F., Jimenez, R., \& Panter, B. 2007, MNRAS, 381, 1252

Trujillo, I., Aguerri, J. A. L., Cepa, J., \& Gutiérrez, C. M. 2001, MNRAS, 328, 977

Valdes, F., Gupta, R., Rose, J. A., Singh, H. P., \& Bell, D. J. 2004, ApJS, 152, 251

van den Bosch, F. C. 2001, MNRAS, 327, 1334

van der Marel, R. P., \& Franx, M. 1993, ApJ, 407, 525

Vazdekis, A., Casuso, E., Peletier, R. F., \& Beckman, J. E. 1996, ApJS, 106, 307 Vazdekis, A., Sánchez-Blázquez, P., Falcón-Barroso, J., et al. 2010, MNRAS, 404, 1639

Walcher, C. J., Wisotzki, L., Bekeraité, S., et al. 2014, A\&A, 569, A1

Worthey, G. 1994, ApJS, 95, 107

Worthey, G., \& Ottaviani, D. L. 1997, ApJS, 111, 377

Yoachim, P., Roškar, R., \& Debattista, V. P. 2012, ApJ, 752, 97

York, D. G., Adelman, J., Anderson, Jr., J. E., et al. 2000, AJ, 120, 1579

Younger, J. D., Cox, T. J., Seth, A. C., \& Hernquist, L. 2007, ApJ, 670, 269

Zhang, H.-X., Hunter, D. A., Elmegreen, B. G., Gao, Y., \& Schruba, A. 2012,

AJ, 143, 47

Zheng, Z., Thilker, D. A., Heckman, T. M., et al. 2015, ApJ, 800, 120

Zibetti, S., Gallazzi, A. R., Ascasibar, Y., et al. 2017, MNRAS, 468, 1902 


\section{Appendix A: SB profile classification}

The proper characterisation of the light distribution in the galaxy sample is an essential step in analysing the stellar content of different SB profiles. Table A.1 summarises the information from the 2D light decomposition presented in this work (see Sect. 3.1). We find that type I $(61.7 \%)$ and II $(32.2 \%)$ are the most frequent SB profiles in the galaxies under analysis with only 13 galaxies $(6.1 \%)$ displaying an upbending profile. Type II galaxies exhibit the most extended inner discs $\left(h_{\text {in }}=8.92 \pm 3.80 \mathrm{kpc}\right)$, followed by type I $(4.88 \pm 1.15 \mathrm{kpc})$ and type III $(2.51 \pm 0.36 \mathrm{kpc})$ discs. The breaks are located at larger galactocentric distances for type II than for type III galaxies $(12.31 \pm 3.25$ versus $8.50 \pm 1.40 \mathrm{kpc}$ ), although in terms of $h_{\text {in }}$ this trend is reversed $\left(1.92 \pm 0.68\right.$ versus $\left.3.51 \pm 0.54 h_{\text {in }}\right)$. There seems to be a tendency with morphology in the inner disc scale length for type I galaxies in which later types show less extended discs than early types. However, no further correlations are found with morphology.

Although it is not the main scope of this paper, for the sake of completeness, in Table A. 2 we compare our results with other works focused on the 1D light distribution up to the outer discs using visible light, i.e. Pohlen \& Trujillo (2006, hereafter PT06), Erwin et al. (2008, hereafter E08), Gutiérrez et al. (2011, hereafter G11), and Marino et al. (2016, hereafter M16). We note that, by no means, is this comparison meant to be complete: there are many more works in the literature to be compared with. We must also highlight the different selection criteria adopted to define each sample. PT06 was focused on analysing the light distribution for late type galaxies (98 galaxies with $2.99<\mathrm{T}<8.49$ ), while E08 and G11 were focused on S0-Sb galaxies (66 barred and 47 unbarred galaxies, respectively). The sample analysed in M16 should, in principle, present more similarities with that analysed in this work as both were drawn from the CALIFA sample (with some overlapping between both samples).

The comparison among these works (including the one presented here) shows some differences. Especially striking is the discrepancy in the frequency of profile types obtained by these studies. While this work suggests that single exponential (type I) profiles are the most frequent type of SB profiles found in our galaxies, PT06, E08, and M16 suggest that galaxies mostly display downbending profiles and upbending SB distributions are most frequently found in G11. However, we find a lack of type III galaxies with the 2D decomposition method. The values for the inner disc scale lengths in all the works suggest that type II galaxies have higher values than type I and III galaxies (in that order), with the exception of G11 for which the order would change to type I, II, and III with decreasing inner disc scale-length values. However, while all the works in 1D found relatively consistent values for this morphological parameter (within errors), the 2D analysis presented here produces larger inner disc scale lengths for type I and II galaxies than the rest of the works. Regarding the break radius, although in physical units different works found different values (both for type II and type III systems), those found for the $R_{\text {break }} / h_{\text {in }}$ for different SB types are fairly consistent among works, with values around 2 for type II galaxies and around 4 for type III systems. All the works seem to suggest that the position of the break in units of $h_{\text {in }}$ is larger for type III galaxies than for downbending profiles while in terms of physical units there is no agreement between them. The tendency found with this $2 \mathrm{D}$ analysis for type I galaxies with later types displaying lower values of $h_{\text {in }}$ is also found when comparing the PT06 results (later types) with the E08 or G11 results (earlier types).

Before drawing any conclusions, we must bear in mind the great discrepancies among the compared works: i) PT06, E08, G11, and M16 follow a 1D analysis of the light distribution while this analysis makes use of a 2D decomposition; in addition; ii) different galaxy samples are analysed in each work; if the samples under analysis are not similar (masses, morphology, etc.) then, different results might arise as a consequence of the differences among the analysed samples.

In order to check to what extent the decomposition method affects the global morphological parameters for type I, II, and III galaxies, we apply a 1D analysis similar to that performed in other works to the sample of galaxies analysed in this work and compare the results from both methods on the same sample. To perform such exercise we have analysed the 1D SB distributions already computed in Sect. 3.2. We fit these 1D SB profiles with a classical exponential decline (Freeman 1970) in the case of type I galaxies, i.e.

$I_{\text {disc }}(r)=I_{0} \mathrm{e}^{-\left(\frac{r}{h_{\text {in }}}\right)}$,

where $I_{\text {disc }}(\mathrm{r})$ is the disc intensity as a function of the radius $(r)$, $I_{0}$ is the central surface brightness, and $h_{\text {in }}$ is the scale length of the disc. For type II and III galaxies we use the function defined in E08 as follows:

$I_{\text {disc }}(r)=S I_{0} \mathrm{e}^{-r / h_{\text {in }}}\left[1+\mathrm{e}^{\alpha\left(r-R_{\text {break }}\right)}\right]^{\frac{1}{\alpha}\left(\frac{1}{h_{\text {in }}}-\frac{1}{h_{\text {out }}}\right)}$,

where $h_{\text {out }}$ is the outer scale length of the disc, $R_{\text {break }}$ is the position of the break, $\alpha$ is a parametrisation of the sharpness of the break, and $S$ is a scaling factor in the form

$S=\left(1+\mathrm{e}^{-\alpha R_{\text {break }}}\right)^{\frac{1}{\alpha}\left(\frac{1}{h_{\text {in }}}-\frac{1}{h_{\text {out }}}\right)}$.

The results of this 1D analysis of the light distribution are shown in Col. (2) of Table A.2. We find again that a discrepancy in the frequency of profile types with this $1 \mathrm{D}$ approach reveals more similar percentages when compared with other works. Not only does the frequency of profile types change from a 1D to a 2D approach, even when analysing the same sample of galaxies, but also the rest of morphological parameters change. Different values for the break radii and inner disc scale lengths are found with no clear pattern. In addition, the comparison of the results from the 1D approach in this sample of galaxies and the results of other works present quite striking differences mainly due to the different samples under analysis.

In summary, the differences among the compared works are mainly due to two aspects. On the one hand, the different decomposition methods used (1D versus 2D) must play an important role in the derived structural parameters; however, a 2D analysis of the light distribution is a more realistic procedure, and thus we decided to follow this approach in this work. On the other hand, the different selection criteria to define each sample and thus, the different morphologies under consideration, might produce different results. It is clear that larger statistical samples and a more rigorous analysis (far from the scope of this present work) are needed to properly assess the variety of SB profiles in nature. These findings are agreement with what has been shown in Méndez-Abreu et al. (2017). 
Table A.1. Surface brightness profile parameters for the sample of galaxies from the 2D decomposition using GASP2D.

\begin{tabular}{|c|c|c|c|c|c|c|c|c|c|}
\hline & $N$ & $h_{\text {in }}(\mathrm{kpc})$ & $R_{\text {break }}(\mathrm{kpc})$ & $R_{\text {break }} / h_{\text {in }}$ & & $N$ & $h_{\text {in }}(\mathrm{kpc})$ & $R_{\text {break }}(\mathrm{kpc})$ & $R_{\text {break }} / h_{\text {in }}$ \\
\hline Type I & $132(61.7 \%)$ & $4.88 \pm 1.15$ & - & - & $\begin{array}{l}\mathrm{Sa} \\
\mathrm{Sb} \\
\mathrm{Sc} \\
\mathrm{Sd} \\
\end{array}$ & $\begin{array}{c}22(10.3) \\
47(22.0) \\
52(24.3) \\
11(5.1) \\
\end{array}$ & $\begin{array}{l}5.22 \pm 1.41 \\
5.22 \pm 0.97 \\
4.66 \pm 1.15 \\
3.75 \pm 1.21 \\
\end{array}$ & $\begin{array}{l}- \\
- \\
- \\
-\end{array}$ & $\begin{array}{l}- \\
- \\
- \\
-\end{array}$ \\
\hline Type II & $69(32.2 \%)$ & $8.92 \pm 3.80$ & $12.31 \pm 3.25$ & $1.92 \pm 0.68$ & $\begin{array}{l}\mathrm{Sa} \\
\mathrm{Sb} \\
\mathrm{Sc} \\
\mathrm{Sd}\end{array}$ & $\begin{array}{c}8(3.7) \\
19(8.9) \\
31(14.5) \\
11(5.1)\end{array}$ & $\begin{array}{c}8.22 \pm 2.55 \\
12.15 \pm 2.61 \\
8.78 \pm 3.15 \\
4.26 \pm 0.85\end{array}$ & $\begin{array}{c}11.09 \pm 2.92 \\
11.77 \pm 2.89 \\
13.86 \pm 3.45 \\
9.77 \pm 2.90\end{array}$ & $\begin{array}{l}2.26 \pm 1.33 \\
1.40 \pm 0.41 \\
2.04 \pm 0.57 \\
2.20 \pm 0.37\end{array}$ \\
\hline Type III & $13(6.1 \%)$ & $2.51 \pm 0.36$ & $8.50 \pm 1.40$ & $3.51 \pm 0.54$ & $\begin{array}{l}\mathrm{Sa} \\
\mathrm{Sb} \\
\mathrm{Sc} \\
\mathrm{Sd}\end{array}$ & $\begin{array}{l}5(2.3) \\
2(0.9) \\
4(2.0) \\
2(0.9)\end{array}$ & $\begin{array}{l}2.98 \pm 0.25 \\
2.55 \pm 0.09 \\
1.89 \pm 0.29 \\
2.51 \pm 0.37\end{array}$ & $\begin{array}{l}9.65 \pm 1.77 \\
7.41 \pm 0.61 \\
8.58 \pm 1.05 \\
6.55 \pm 0.78\end{array}$ & $\begin{array}{l}7.17 \pm 0.45 \\
2.89 \pm 0.14 \\
4.67 \pm 0.40 \\
2.62 \pm 0.09\end{array}$ \\
\hline
\end{tabular}

Notes. See Sect. 3.1 for further details.

Table A.2. Comparison between the surface brightness profile parameters presented here and other works.

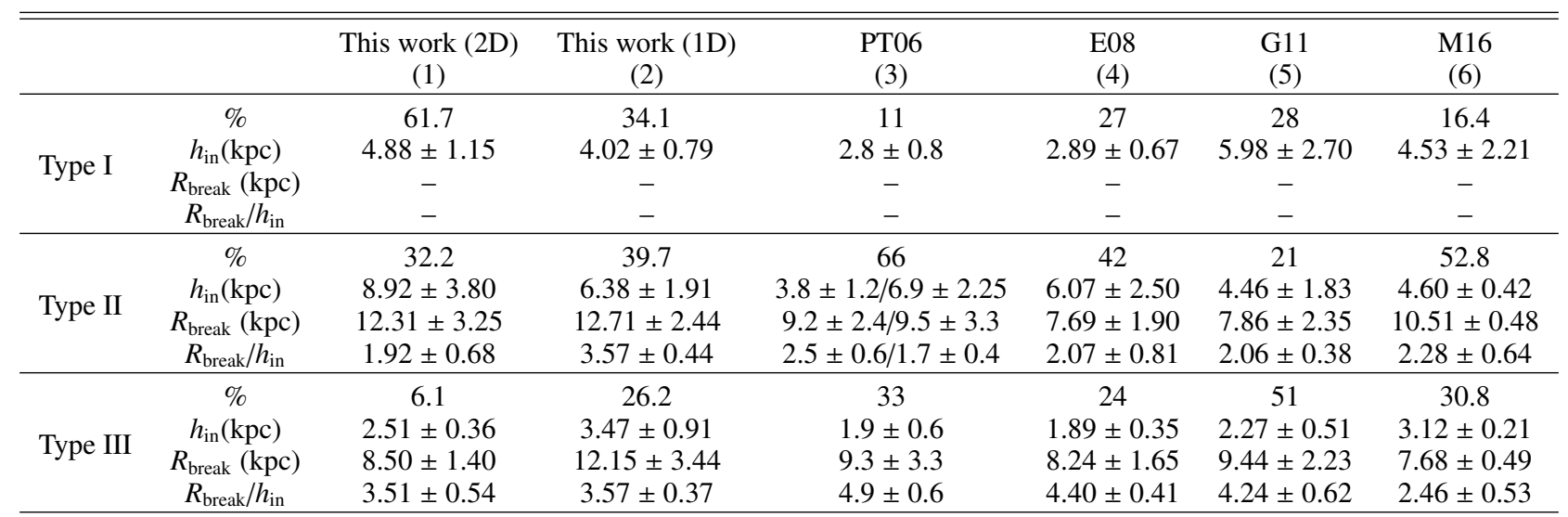

Notes. (1) Results applying the 2D decomposition using GASP2D (see Sect. 3.1 for further details) to the sample of galaxies under analysis; (2) results applying a more classical 1D approach (see text for details) to the sample of galaxies; (3) results from Pohlen \& Trujillo (2006); for type II galaxies the parameters are divided into classical truncations and breaks associated with the Outer Lindblad Reso-ce (II-CT/II-o.OLR); (4) results from Erwin et al. (2008); (5) results from Gutiérrez et al. (2011); and (6) results from Marino et al. (2016). 
T. Ruiz-Lara et al.: Observational hints of radial migration in disc galaxies from CALIFA

\section{Appendix B: Stellar parameter and colour gradients}

In this section we present tables with the derived stellar age and metallicity profile inner gradients as well as colour profile inner gradients for all the galaxies in the sample. Table B.1 lists this information for the stellar age profiles, Table B.2 for the [M/H] profiles, and Table B. 3 for the colour $(g-r, g-i$, and $r-i)$ profiles.

Table B.1. Stellar age inner gradients from the stellar content analysis (see Sect. 4).

\begin{tabular}{|c|c|c|c|c|}
\hline $\begin{array}{l}\text { Name } \\
\text { (1) }\end{array}$ & $\begin{array}{r}\nabla \log \\
\left(\operatorname{dex} / h_{\text {in }}\right) \\
(2)\end{array}$ & $\begin{array}{r}\text { (dex/kpc) } \\
\text { (3),in } \\
\end{array}$ & $\begin{array}{r}\nabla \log \\
\left(\operatorname{dex} / h_{\text {in }}\right) \\
(4)\end{array}$ & $\begin{array}{l})_{\mathrm{M}, \mathrm{in}} \\
\quad(\mathrm{dex} / \mathrm{kpc}) \\
\end{array}$ \\
\hline UGC 00005 & $-0.122 \pm 0.008$ & $-0.019 \pm 0.001$ & $-0.008 \pm 0.005$ & $-0.001 \pm 0.001$ \\
\hline NGC 7819 & $-0.098 \pm 0.025$ & $-0.006 \pm 0.002$ & $-0.184 \pm 0.016$ & $-0.012 \pm 0.001$ \\
\hline IC 1528 & $-0.138 \pm 0.007$ & $-0.033 \pm 0.002$ & $-0.053 \pm 0.004$ & $-0.013 \pm 0.001$ \\
\hline UGC 00036 & $-0.207 \pm 0.008$ & $-0.051 \pm 0.002$ & $-0.016 \pm 0.005$ & $-0.004 \pm 0.001$ \\
\hline NGC 0001 & $-0.254 \pm 0.007$ & $-0.059 \pm 0.002$ & $-0.001 \pm 0.005$ & $-0.0 \pm 0.001$ \\
\hline NGC 0023 & $1.404 \pm 0.116$ & $0.041 \pm 0.003$ & $0.267 \pm 0.072$ & $0.008 \pm 0.002$ \\
\hline NGC 0036 & $-0.414 \pm 0.008$ & $-0.043 \pm 0.001$ & $-0.024 \pm 0.005$ & $-0.003 \pm 0.001$ \\
\hline UGC 00139 & $0.005 \pm 0.077$ & $0.001 \pm 0.024$ & $-0.04 \pm 0.036$ & $-0.012 \pm 0.011$ \\
\hline MCG-02-02-030 & $-0.703 \pm 0.04$ & $-0.156 \pm 0.009$ & $0.049 \pm 0.014$ & $0.011 \pm 0.003$ \\
\hline UGC 00312 & $-0.046 \pm 0.041$ & $-0.008 \pm 0.007$ & $-0.065 \pm 0.015$ & $-0.012 \pm 0.003$ \\
\hline ESO540-G003 & $-0.897 \pm 0.033$ & $-0.265 \pm 0.01$ & $-0.161 \pm 0.024$ & $-0.047 \pm 0.007$ \\
\hline NGC 0160 & $-0.769 \pm 0.034$ & $-0.045 \pm 0.002$ & $-0.086 \pm 0.022$ & $-0.005 \pm 0.001$ \\
\hline NGC 0165 & $-0.072 \pm 0.013$ & $-0.012 \pm 0.002$ & $-0.018 \pm 0.012$ & $-0.003 \pm 0.002$ \\
\hline NGC 0171 & $-2.063 \pm 0.037$ & $-0.101 \pm 0.002$ & $-0.336 \pm 0.019$ & $-0.016 \pm 0.001$ \\
\hline NGC 0180 & $-0.455 \pm 0.008$ & $-0.054 \pm 0.001$ & $0.018 \pm 0.005$ & $0.002 \pm 0.001$ \\
\hline NGC 0214 & $-0.343 \pm 0.015$ & $-0.08 \pm 0.003$ & $-0.004 \pm 0.008$ & $-0.001 \pm 0.002$ \\
\hline NGC 0237 & $-0.002 \pm 0.007$ & $-0.001 \pm 0.003$ & $-0.036 \pm 0.005$ & $-0.013 \pm 0.002$ \\
\hline NGC 0234 & $-0.1 \pm 0.013$ & $-0.018 \pm 0.002$ & $-0.071 \pm 0.019$ & $-0.013 \pm 0.003$ \\
\hline NGC 0257 & $-0.173 \pm 0.008$ & $-0.031 \pm 0.002$ & $-0.066 \pm 0.008$ & $-0.012 \pm 0.001$ \\
\hline NGC 0309 & $-0.434 \pm 0.014$ & $-0.047 \pm 0.002$ & $-0.086 \pm 0.006$ & $-0.009 \pm 0.001$ \\
\hline NGC 0447 & $-0.426 \pm 0.023$ & $-0.042 \pm 0.002$ & $-0.045 \pm 0.015$ & $-0.004 \pm 0.002$ \\
\hline NGC 0477 & $-0.395 \pm 0.008$ & $-0.058 \pm 0.001$ & $-0.13 \pm 0.005$ & $-0.019 \pm 0.001$ \\
\hline IC 1683 & $0.269 \pm 0.009$ & $0.055 \pm 0.002$ & $0.061 \pm 0.006$ & $0.012 \pm 0.001$ \\
\hline NGC 0496 & $-0.008 \pm 0.01$ & $-0.002 \pm 0.002$ & $-0.107 \pm 0.006$ & $-0.019 \pm 0.001$ \\
\hline NGC 0528 & $-0.038 \pm 0.004$ & $-0.013 \pm 0.001$ & $-0.016 \pm 0.003$ & $-0.006 \pm 0.001$ \\
\hline NGC 0551 & $-0.17 \pm 0.007$ & $-0.033 \pm 0.001$ & $0.01 \pm 0.003$ & $0.002 \pm 0.001$ \\
\hline NGC 0570 & $-0.185 \pm 0.01$ & $-0.033 \pm 0.002$ & $-0.067 \pm 0.005$ & $-0.012 \pm 0.001$ \\
\hline UGC 01271 & $-0.017 \pm 0.008$ & $-0.004 \pm 0.002$ & $0.032 \pm 0.006$ & $0.008 \pm 0.001$ \\
\hline NGC 0716 & $-0.063 \pm 0.011$ & $-0.019 \pm 0.003$ & $-0.055 \pm 0.006$ & $-0.016 \pm 0.002$ \\
\hline NGC 0768 & $-0.053 \pm 0.012$ & $-0.008 \pm 0.002$ & $0.011 \pm 0.01$ & $0.002 \pm 0.002$ \\
\hline NGC 0776 & $-0.259 \pm 0.008$ & $-0.046 \pm 0.001$ & $0.019 \pm 0.005$ & $0.003 \pm 0.001$ \\
\hline NGC 0787 & $-0.198 \pm 0.015$ & $-0.049 \pm 0.004$ & $-0.031 \pm 0.008$ & $-0.008 \pm 0.002$ \\
\hline NGC 0842 & $-0.024 \pm 0.006$ & $-0.005 \pm 0.001$ & $0.026 \pm 0.005$ & $0.006 \pm 0.001$ \\
\hline UGC 01659 & $-1.17 \pm 0.099$ & $-0.039 \pm 0.003$ & $-0.627 \pm 0.065$ & $-0.021 \pm 0.002$ \\
\hline NGC 0873 & $0.082 \pm 0.007$ & $0.03 \pm 0.002$ & $-0.053 \pm 0.006$ & $-0.019 \pm 0.002$ \\
\hline NGC 0924 & $0.124 \pm 0.037$ & $0.023 \pm 0.007$ & $0.034 \pm 0.012$ & $0.006 \pm 0.002$ \\
\hline UGC 01918 & $-0.224 \pm 0.033$ & $-0.036 \pm 0.005$ & $0.056 \pm 0.017$ & $0.009 \pm 0.003$ \\
\hline NGC 0932 & $-0.203 \pm 0.008$ & $-0.037 \pm 0.002$ & $-0.036 \pm 0.005$ & $-0.007 \pm 0.001$ \\
\hline NGC 0941 & $-0.186 \pm 0.015$ & $-0.083 \pm 0.007$ & $-0.096 \pm 0.01$ & $-0.043 \pm 0.005$ \\
\hline NGC 0976 & $-0.325 \pm 0.045$ & $-0.098 \pm 0.014$ & $0.115 \pm 0.024$ & $0.035 \pm 0.007$ \\
\hline NGC 0991 & $-0.504 \pm 0.013$ & $-0.173 \pm 0.005$ & $-0.039 \pm 0.008$ & $-0.014 \pm 0.003$ \\
\hline UGC 02099 & $-0.343 \pm 0.021$ & $-0.033 \pm 0.002$ & $-0.08 \pm 0.012$ & $-0.008 \pm 0.001$ \\
\hline UGC 02134 & $-0.894 \pm 0.078$ & $-0.042 \pm 0.004$ & $-0.073 \pm 0.046$ & $-0.003 \pm 0.002$ \\
\hline NGC 1070 & $-0.149 \pm 0.008$ & $-0.063 \pm 0.003$ & $0.016 \pm 0.004$ & $0.007 \pm 0.002$ \\
\hline NGC 1094 & $-0.186 \pm 0.01$ & $-0.069 \pm 0.004$ & $-0.105 \pm 0.012$ & $-0.039 \pm 0.004$ \\
\hline NGC 1093 & $-0.164 \pm 0.009$ & $-0.036 \pm 0.002$ & $-0.026 \pm 0.005$ & $-0.006 \pm 0.001$ \\
\hline UGC 02311 & $-0.094 \pm 0.011$ & $-0.016 \pm 0.002$ & $-0.066 \pm 0.011$ & $-0.012 \pm 0.002$ \\
\hline UGC 02403 & $0.32 \pm 0.041$ & $0.065 \pm 0.008$ & $0.034 \pm 0.028$ & $0.007 \pm 0.006$ \\
\hline UGC 02443 & $-0.322 \pm 0.026$ & $-0.087 \pm 0.007$ & $-0.095 \pm 0.017$ & $-0.026 \pm 0.005$ \\
\hline NGC 1167 & $-0.248 \pm 0.013$ & $-0.028 \pm 0.002$ & $-0.099 \pm 0.009$ & $-0.011 \pm 0.001$ \\
\hline NGC 1211 & $-0.124 \pm 0.056$ & $-0.019 \pm 0.009$ & $-0.075 \pm 0.033$ & $-0.012 \pm 0.005$ \\
\hline UGC 02690 & $-0.013 \pm 0.034$ & $-0.003 \pm 0.007$ & $-0.102 \pm 0.014$ & $-0.021 \pm 0.003$ \\
\hline MCG-01-10-019 & $-0.149 \pm 0.013$ & $-0.021 \pm 0.002$ & $-0.135 \pm 0.007$ & $-0.019 \pm 0.001$ \\
\hline NGC 1645 & $-0.329 \pm 0.007$ & $-0.062 \pm 0.001$ & $-0.071 \pm 0.004$ & $-0.013 \pm 0.001$ \\
\hline NGC 1659 & $-0.05 \pm 0.008$ & $-0.014 \pm 0.002$ & $-0.032 \pm 0.005$ & $-0.009 \pm 0.001$ \\
\hline
\end{tabular}

Notes. This table shows the gradients for the light- and mass-weighted $\log (\mathrm{Age})\left(\mathrm{dex} / h_{\mathrm{in}}\right.$ and dex/kpc) represented in Fig. 6 . 
Table B.1. continued.

\begin{tabular}{|c|c|c|c|c|}
\hline $\begin{array}{l}\text { Name } \\
\text { (1) }\end{array}$ & $\begin{array}{r}\nabla \log \\
\left(\operatorname{dex} / h_{\text {in }}\right) \\
(2)\end{array}$ & $\begin{array}{l}\text { e) })_{\mathrm{L}, \text { in }} \\
\quad(\operatorname{dex} / \mathrm{kpc}) \\
(3)\end{array}$ & $\begin{array}{r}\nabla \log \\
\left(\operatorname{dex} / h_{\text {in }}\right) \\
(4)\end{array}$ & $\begin{array}{r})_{\mathrm{M}, \text { in }} \\
\quad(\mathrm{dex} / \mathrm{kpc}) \\
(5)\end{array}$ \\
\hline NGC 1665 & $-0.023 \pm 0.006$ & $-0.007 \pm 0.002$ & $0.004 \pm 0.004$ & $0.001 \pm 0.001$ \\
\hline NGC 1666 & $-0.051 \pm 0.008$ & $-0.02 \pm 0.003$ & $0.031 \pm 0.006$ & $0.012 \pm 0.002$ \\
\hline NGC 1667 & $-0.089 \pm 0.006$ & $-0.025 \pm 0.002$ & $-0.035 \pm 0.004$ & $-0.01 \pm 0.001$ \\
\hline UGC 03253 & $-0.499 \pm 0.012$ & $-0.139 \pm 0.003$ & $-0.063 \pm 0.007$ & $-0.017 \pm 0.002$ \\
\hline NGC 2253 & $-0.182 \pm 0.007$ & $-0.063 \pm 0.002$ & $-0.028 \pm 0.005$ & $-0.01 \pm 0.002$ \\
\hline NGC 2347 & $-0.286 \pm 0.008$ & $-0.061 \pm 0.002$ & $-0.025 \pm 0.006$ & $-0.005 \pm 0.001$ \\
\hline UGC 03944 & $-0.198 \pm 0.013$ & $-0.063 \pm 0.004$ & $-0.113 \pm 0.012$ & $-0.036 \pm 0.004$ \\
\hline UGC 03973 & $-0.002 \pm 0.012$ & $-0.0 \pm 0.002$ & $-0.08 \pm 0.008$ & $-0.011 \pm 0.001$ \\
\hline UGC 03995 & $-0.412 \pm 0.011$ & $-0.056 \pm 0.001$ & $-0.008 \pm 0.005$ & $-0.001 \pm 0.001$ \\
\hline NGC 2449 & $-0.134 \pm 0.006$ & $-0.03 \pm 0.001$ & $0.011 \pm 0.003$ & $0.002 \pm 0.001$ \\
\hline NGC 2487 & $-0.785 \pm 0.012$ & $-0.106 \pm 0.002$ & $-0.067 \pm 0.009$ & $-0.009 \pm 0.001$ \\
\hline UGC 04195 & $-0.041 \pm 0.039$ & $-0.006 \pm 0.006$ & $0.001 \pm 0.01$ & $0.0 \pm 0.001$ \\
\hline NGC 2530 & $-0.078 \pm 0.015$ & $-0.017 \pm 0.003$ & $-0.1 \pm 0.01$ & $-0.022 \pm 0.002$ \\
\hline NGC 2540 & $-0.242 \pm 0.01$ & $-0.045 \pm 0.002$ & $-0.113 \pm 0.011$ & $-0.021 \pm 0.002$ \\
\hline NGC 2543 & $-0.467 \pm 0.009$ & $-0.123 \pm 0.002$ & $0.012 \pm 0.008$ & $0.003 \pm 0.002$ \\
\hline UGC 04308 & $-0.349 \pm 0.011$ & $-0.083 \pm 0.003$ & $-0.086 \pm 0.008$ & $-0.021 \pm 0.002$ \\
\hline NGC 2553 & $-0.14 \pm 0.007$ & $-0.032 \pm 0.002$ & $-0.068 \pm 0.005$ & $-0.016 \pm 0.001$ \\
\hline UGC 04262 & $-0.307 \pm 0.012$ & $-0.053 \pm 0.002$ & $-0.129 \pm 0.007$ & $-0.022 \pm 0.001$ \\
\hline NGC 2558 & $-0.461 \pm 0.01$ & $-0.081 \pm 0.002$ & $-0.128 \pm 0.007$ & $-0.023 \pm 0.001$ \\
\hline NGC 2565 & $-0.184 \pm 0.007$ & $-0.052 \pm 0.002$ & $0.108 \pm 0.005$ & $0.03 \pm 0.001$ \\
\hline NGC 2572 & $-0.156 \pm 0.008$ & $-0.032 \pm 0.002$ & $0.023 \pm 0.004$ & $0.005 \pm 0.001$ \\
\hline UGC 04375 & $-0.392 \pm 0.007$ & $-0.144 \pm 0.003$ & $-0.044 \pm 0.005$ & $-0.016 \pm 0.002$ \\
\hline NGC 2596 & $-0.494 \pm 0.018$ & $-0.056 \pm 0.002$ & $-0.155 \pm 0.016$ & $-0.017 \pm 0.002$ \\
\hline NGC 2595 & $-0.503 \pm 0.015$ & $-0.058 \pm 0.002$ & $-0.059 \pm 0.014$ & $-0.007 \pm 0.002$ \\
\hline NGC 2604 & $-0.245 \pm 0.021$ & $-0.083 \pm 0.007$ & $-0.105 \pm 0.009$ & $-0.036 \pm 0.003$ \\
\hline NGC 2639 & $-0.01 \pm 0.003$ & $-0.004 \pm 0.001$ & $0.028 \pm 0.002$ & $0.01 \pm 0.001$ \\
\hline NGC 2730 & $-0.181 \pm 0.02$ & $-0.028 \pm 0.003$ & $-0.194 \pm 0.011$ & $-0.03 \pm 0.002$ \\
\hline NGC 2805 & $-1.887 \pm 0.233$ & $-0.275 \pm 0.034$ & $-1.143 \pm 0.153$ & $-0.167 \pm 0.022$ \\
\hline NGC 2906 & $-0.136 \pm 0.004$ & $-0.068 \pm 0.002$ & $0.042 \pm 0.002$ & $0.021 \pm 0.001$ \\
\hline NGC 2916 & $-0.728 \pm 0.01$ & $-0.129 \pm 0.002$ & $-0.052 \pm 0.007$ & $-0.009 \pm 0.001$ \\
\hline UGC 05108 & $-0.751 \pm 0.054$ & $-0.019 \pm 0.001$ & $0.114 \pm 0.028$ & $0.003 \pm 0.001$ \\
\hline UGC 05359 & $-0.171 \pm 0.008$ & $-0.026 \pm 0.001$ & $-0.08 \pm 0.004$ & $-0.012 \pm 0.001$ \\
\hline UGC 05396 & $-0.41 \pm 0.013$ & $-0.064 \pm 0.002$ & $-0.012 \pm 0.008$ & $-0.002 \pm 0.001$ \\
\hline NGC 3106 & $-0.312 \pm 0.011$ & $-0.041 \pm 0.001$ & $-0.073 \pm 0.008$ & $-0.01 \pm 0.001$ \\
\hline NGC 3300 & $0.01 \pm 0.006$ & $0.004 \pm 0.002$ & $0.029 \pm 0.005$ & $0.01 \pm 0.002$ \\
\hline NGC 3381 & $-0.036 \pm 0.009$ & $-0.018 \pm 0.004$ & $-0.005 \pm 0.005$ & $-0.002 \pm 0.003$ \\
\hline IC 0674 & $-0.141 \pm 0.008$ & $-0.022 \pm 0.001$ & $0.03 \pm 0.004$ & $0.005 \pm 0.001$ \\
\hline UGC 06312 & $-0.004 \pm 0.012$ & $-0.001 \pm 0.002$ & $0.062 \pm 0.009$ & $0.009 \pm 0.001$ \\
\hline NGC 3614 & $-0.124 \pm 0.022$ & $-0.026 \pm 0.005$ & $0.059 \pm 0.014$ & $0.012 \pm 0.003$ \\
\hline NGC 3687 & $-0.189 \pm 0.01$ & $-0.083 \pm 0.004$ & $0.004 \pm 0.006$ & $0.002 \pm 0.002$ \\
\hline NGC 3811 & $-0.171 \pm 0.007$ & $-0.066 \pm 0.003$ & $-0.069 \pm 0.003$ & $-0.027 \pm 0.001$ \\
\hline NGC 3815 & $-0.077 \pm 0.005$ & $-0.027 \pm 0.002$ & $-0.004 \pm 0.003$ & $-0.001 \pm 0.001$ \\
\hline NGC 3994 & $-0.075 \pm 0.008$ & $-0.059 \pm 0.007$ & $0.004 \pm 0.005$ & $0.003 \pm 0.004$ \\
\hline NGC 4003 & $-0.204 \pm 0.009$ & $-0.031 \pm 0.001$ & $-0.017 \pm 0.005$ & $-0.003 \pm 0.001$ \\
\hline UGC 07012 & $-0.133 \pm 0.018$ & $-0.076 \pm 0.01$ & $-0.064 \pm 0.008$ & $-0.037 \pm 0.004$ \\
\hline NGC 4047 & $-0.037 \pm 0.009$ & $-0.015 \pm 0.004$ & $-0.014 \pm 0.006$ & $-0.006 \pm 0.002$ \\
\hline UGC 07145 & $-0.441 \pm 0.013$ & $-0.07 \pm 0.002$ & $-0.045 \pm 0.007$ & $-0.007 \pm 0.001$ \\
\hline NGC 4185 & $-0.404 \pm 0.011$ & $-0.054 \pm 0.001$ & $-0.087 \pm 0.006$ & $-0.012 \pm 0.001$ \\
\hline NGC 4210 & $-0.344 \pm 0.017$ & $-0.06 \pm 0.003$ & $0.059 \pm 0.011$ & $0.01 \pm 0.002$ \\
\hline NGC 4470 & $0.124 \pm 0.008$ & $0.07 \pm 0.004$ & $0.003 \pm 0.005$ & $0.002 \pm 0.003$ \\
\hline NGC 4711 & $-0.325 \pm 0.011$ & $-0.082 \pm 0.003$ & $-0.017 \pm 0.006$ & $-0.004 \pm 0.002$ \\
\hline UGC 08004 & $-0.072 \pm 0.018$ & $-0.011 \pm 0.003$ & $-0.017 \pm 0.01$ & $-0.003 \pm 0.002$ \\
\hline NGC 4961 & $-0.099 \pm 0.023$ & $-0.039 \pm 0.009$ & $0.01 \pm 0.012$ & $0.004 \pm 0.005$ \\
\hline UGC 08231 & $-0.17 \pm 0.011$ & $-0.075 \pm 0.005$ & $-0.013 \pm 0.006$ & $-0.006 \pm 0.002$ \\
\hline NGC 5000 & $-0.305 \pm 0.009$ & $-0.055 \pm 0.002$ & $-0.109 \pm 0.007$ & $-0.019 \pm 0.001$ \\
\hline NGC 5016 & $-0.108 \pm 0.008$ & $-0.054 \pm 0.004$ & $-0.022 \pm 0.005$ & $-0.011 \pm 0.002$ \\
\hline NGC 5157 & $-0.334 \pm 0.007$ & $-0.046 \pm 0.001$ & $-0.065 \pm 0.004$ & $-0.009 \pm 0.001$ \\
\hline NGC 5205 & $-0.474 \pm 0.009$ & $-0.267 \pm 0.005$ & $-0.046 \pm 0.005$ & $-0.026 \pm 0.003$ \\
\hline NGC 5267 & $-0.51 \pm 0.013$ & $-0.052 \pm 0.001$ & $0.014 \pm 0.008$ & $0.001 \pm 0.001$ \\
\hline NGC 5320 & $-0.256 \pm 0.009$ & $-0.068 \pm 0.002$ & $0.028 \pm 0.006$ & $0.007 \pm 0.002$ \\
\hline UGC 08781 & $-0.278 \pm 0.009$ & $-0.036 \pm 0.001$ & $-0.029 \pm 0.006$ & $-0.004 \pm 0.001$ \\
\hline NGC 5376 & $-0.259 \pm 0.01$ & $-0.088 \pm 0.004$ & $0.017 \pm 0.006$ & $0.006 \pm 0.002$ \\
\hline NGC 5379 & $0.035 \pm 0.007$ & $0.019 \pm 0.004$ & $-0.036 \pm 0.004$ & $-0.02 \pm 0.002$ \\
\hline
\end{tabular}


T. Ruiz-Lara et al.: Observational hints of radial migration in disc galaxies from CALIFA

Table B.1. continued.

\begin{tabular}{|c|c|c|c|c|}
\hline $\begin{array}{l}\text { Name } \\
\text { (1) }\end{array}$ & $\begin{array}{r}\nabla \log \\
\left(\operatorname{dex} / h_{\text {in }}\right) \\
(2) \\
\end{array}$ & $\begin{array}{r}e_{\mathrm{L}, \text { in }} \\
\quad(\mathrm{dex} / \mathrm{kpc}) \\
(3) \\
\end{array}$ & $\begin{array}{r}\nabla \log \\
\left(\operatorname{dex} / h_{\text {in }}\right) \\
(4) \\
\end{array}$ & $\begin{array}{r})_{\mathrm{M}, \mathrm{in}} \\
\quad(\mathrm{dex} / \mathrm{kpc}) \\
(5) \\
\end{array}$ \\
\hline NGC 5378 & $-0.422 \pm 0.008$ & $-0.082 \pm 0.002$ & $-0.123 \pm 0.006$ & $-0.024 \pm 0.001$ \\
\hline NGC 5406 & $-1.79 \pm 0.041$ & $-0.081 \pm 0.002$ & $-0.093 \pm 0.02$ & $-0.004 \pm 0.001$ \\
\hline NGC 5473 & $0.047 \pm 0.008$ & $0.02 \pm 0.003$ & $0.03 \pm 0.006$ & $0.013 \pm 0.002$ \\
\hline NGC 5480 & $-0.116 \pm 0.019$ & $-0.039 \pm 0.007$ & $-0.082 \pm 0.017$ & $-0.028 \pm 0.006$ \\
\hline UGC 09067 & $-0.115 \pm 0.009$ & $-0.025 \pm 0.002$ & $-0.008 \pm 0.006$ & $-0.002 \pm 0.001$ \\
\hline NGC 5520 & $-0.112 \pm 0.005$ & $-0.068 \pm 0.003$ & $-0.07 \pm 0.005$ & $-0.042 \pm 0.003$ \\
\hline NGC 5633 & $-0.086 \pm 0.006$ & $-0.061 \pm 0.004$ & $-0.042 \pm 0.004$ & $-0.03 \pm 0.003$ \\
\hline NGC 5657 & $-0.178 \pm 0.006$ & $-0.045 \pm 0.002$ & $-0.009 \pm 0.003$ & $-0.002 \pm 0.001$ \\
\hline NGC 5720 & $-0.372 \pm 0.007$ & $-0.055 \pm 0.001$ & $-0.061 \pm 0.004$ & $-0.009 \pm 0.001$ \\
\hline NGC 5732 & $0.025 \pm 0.008$ & $0.01 \pm 0.003$ & $-0.081 \pm 0.008$ & $-0.031 \pm 0.003$ \\
\hline UGC 09476 & $-0.203 \pm 0.009$ & $-0.052 \pm 0.002$ & $-0.147 \pm 0.007$ & $-0.037 \pm 0.002$ \\
\hline NGC 5784 & $0.065 \pm 0.011$ & $0.01 \pm 0.002$ & $-0.024 \pm 0.007$ & $-0.004 \pm 0.001$ \\
\hline UGC 09598 & $-0.437 \pm 0.01$ & $-0.074 \pm 0.002$ & $-0.004 \pm 0.006$ & $-0.001 \pm 0.001$ \\
\hline NGC 5876 & $-0.487 \pm 0.011$ & $-0.068 \pm 0.002$ & $-0.109 \pm 0.006$ & $-0.015 \pm 0.001$ \\
\hline NGC 5888 & $-0.112 \pm 0.011$ & $-0.011 \pm 0.001$ & $-0.029 \pm 0.005$ & $-0.003 \pm 0.0$ \\
\hline UGC 09777 & $-0.222 \pm 0.009$ & $-0.089 \pm 0.004$ & $-0.078 \pm 0.006$ & $-0.031 \pm 0.002$ \\
\hline UGC 09842 & $-0.09 \pm 0.013$ & $-0.013 \pm 0.002$ & $-0.069 \pm 0.008$ & $-0.01 \pm 0.001$ \\
\hline NGC 5957 & $-0.452 \pm 0.007$ & $-0.179 \pm 0.003$ & $-0.064 \pm 0.005$ & $-0.025 \pm 0.002$ \\
\hline NGC 5971 & $-0.619 \pm 0.03$ & $-0.146 \pm 0.007$ & $-0.137 \pm 0.019$ & $-0.032 \pm 0.005$ \\
\hline IC 4566 & $-0.264 \pm 0.012$ & $-0.055 \pm 0.003$ & $0.005 \pm 0.007$ & $0.001 \pm 0.001$ \\
\hline NGC 5980 & $-0.189 \pm 0.007$ & $-0.056 \pm 0.002$ & $-0.02 \pm 0.007$ & $-0.006 \pm 0.002$ \\
\hline NGC 6004 & $-0.168 \pm 0.007$ & $-0.035 \pm 0.001$ & $0.013 \pm 0.003$ & $0.003 \pm 0.001$ \\
\hline IC 1151 & $-0.128 \pm 0.01$ & $-0.049 \pm 0.004$ & $-0.081 \pm 0.005$ & $-0.031 \pm 0.002$ \\
\hline NGC 6032 & $-2.477 \pm 0.099$ & $-0.084 \pm 0.003$ & $-0.753 \pm 0.058$ & $-0.025 \pm 0.002$ \\
\hline NGC 6060 & $-0.321 \pm 0.009$ & $-0.048 \pm 0.001$ & $-0.041 \pm 0.005$ & $-0.006 \pm 0.001$ \\
\hline NGC 6063 & $-0.532 \pm 0.017$ & $-0.126 \pm 0.004$ & $-0.072 \pm 0.012$ & $-0.017 \pm 0.003$ \\
\hline IC 1199 & $-0.362 \pm 0.016$ & $-0.062 \pm 0.003$ & $0.019 \pm 0.013$ & $0.003 \pm 0.002$ \\
\hline NGC 6154 & $-0.524 \pm 0.01$ & $-0.075 \pm 0.001$ & $-0.086 \pm 0.005$ & $-0.012 \pm 0.001$ \\
\hline NGC 6155 & $-0.076 \pm 0.006$ & $-0.039 \pm 0.003$ & $-0.123 \pm 0.005$ & $-0.063 \pm 0.003$ \\
\hline NGC 6186 & $-0.245 \pm 0.02$ & $-0.03 \pm 0.002$ & $-0.216 \pm 0.023$ & $-0.027 \pm 0.003$ \\
\hline NGC 6278 & $-0.132 \pm 0.009$ & $-0.028 \pm 0.002$ & $-0.076 \pm 0.006$ & $-0.016 \pm 0.001$ \\
\hline NGC 6301 & $-0.534 \pm 0.015$ & $-0.044 \pm 0.001$ & $-0.086 \pm 0.009$ & $-0.007 \pm 0.001$ \\
\hline NGC 6314 & $0.061 \pm 0.009$ & $0.009 \pm 0.001$ & $0.022 \pm 0.008$ & $0.003 \pm 0.001$ \\
\hline UGC 10796 & $0.188 \pm 0.021$ & $0.024 \pm 0.003$ & $-0.002 \pm 0.01$ & $-0.0 \pm 0.001$ \\
\hline UGC 10811 & $-0.368 \pm 0.01$ & $-0.052 \pm 0.001$ & $-0.015 \pm 0.005$ & $-0.002 \pm 0.001$ \\
\hline IC 1256 & $-0.182 \pm 0.009$ & $-0.051 \pm 0.002$ & $-0.108 \pm 0.007$ & $-0.03 \pm 0.002$ \\
\hline NGC 6394 & $-0.049 \pm 0.009$ & $-0.007 \pm 0.001$ & $-0.056 \pm 0.006$ & $-0.008 \pm 0.001$ \\
\hline UGC 10905 & $0.016 \pm 0.012$ & $0.001 \pm 0.001$ & $-0.036 \pm 0.008$ & $-0.003 \pm 0.001$ \\
\hline NGC 6427 & $-0.033 \pm 0.005$ & $-0.011 \pm 0.002$ & $0.005 \pm 0.003$ & $0.002 \pm 0.001$ \\
\hline NGC 6478 & $-0.463 \pm 0.018$ & $-0.048 \pm 0.002$ & $-0.057 \pm 0.011$ & $-0.006 \pm 0.001$ \\
\hline NGC 6497 & $-0.763 \pm 0.015$ & $-0.09 \pm 0.002$ & $-0.147 \pm 0.007$ & $-0.017 \pm 0.001$ \\
\hline UGC 11228 & $-0.075 \pm 0.011$ & $-0.014 \pm 0.002$ & $-0.018 \pm 0.007$ & $-0.003 \pm 0.001$ \\
\hline UGC 11262 & $-0.281 \pm 0.015$ & $-0.042 \pm 0.002$ & $-0.125 \pm 0.009$ & $-0.019 \pm 0.001$ \\
\hline MCG-02-51-004 & $-0.224 \pm 0.014$ & $-0.043 \pm 0.003$ & $-0.015 \pm 0.008$ & $-0.003 \pm 0.002$ \\
\hline NGC 6941 & $-0.56 \pm 0.01$ & $-0.074 \pm 0.001$ & $-0.09 \pm 0.006$ & $-0.012 \pm 0.001$ \\
\hline NGC 6945 & $-0.064 \pm 0.008$ & $-0.015 \pm 0.002$ & $-0.025 \pm 0.005$ & $-0.006 \pm 0.001$ \\
\hline NGC 6978 & $-0.069 \pm 0.007$ & $-0.012 \pm 0.001$ & $-0.041 \pm 0.004$ & $-0.007 \pm 0.001$ \\
\hline UGC 11649 & $-0.528 \pm 0.01$ & $-0.132 \pm 0.002$ & $-0.149 \pm 0.006$ & $-0.037 \pm 0.002$ \\
\hline NGC 7047 & $-0.342 \pm 0.027$ & $-0.05 \pm 0.004$ & $-0.063 \pm 0.011$ & $-0.009 \pm 0.002$ \\
\hline NGC 7311 & $-0.186 \pm 0.005$ & $-0.051 \pm 0.002$ & $0.021 \pm 0.004$ & $0.006 \pm 0.001$ \\
\hline NGC 7321 & $-0.185 \pm 0.006$ & $-0.034 \pm 0.001$ & $0.061 \pm 0.007$ & $0.011 \pm 0.001$ \\
\hline UGC 12185 & $-0.586 \pm 0.018$ & $-0.047 \pm 0.001$ & $-0.341 \pm 0.018$ & $-0.028 \pm 0.001$ \\
\hline UGC 12224 & $-0.255 \pm 0.015$ & $-0.055 \pm 0.003$ & $-0.095 \pm 0.01$ & $-0.021 \pm 0.002$ \\
\hline NGC 7466 & $-0.191 \pm 0.012$ & $-0.025 \pm 0.002$ & $-0.068 \pm 0.01$ & $-0.009 \pm 0.001$ \\
\hline NGC 7489 & $-0.214 \pm 0.014$ & $-0.039 \pm 0.003$ & $-0.136 \pm 0.008$ & $-0.025 \pm 0.001$ \\
\hline NGC 7536 & $-0.511 \pm 0.084$ & $-0.059 \pm 0.01$ & $-0.085 \pm 0.062$ & $-0.01 \pm 0.007$ \\
\hline NGC 7549 & $-0.368 \pm 0.016$ & $-0.045 \pm 0.002$ & $-0.071 \pm 0.008$ & $-0.009 \pm 0.001$ \\
\hline NGC 7563 & $-0.366 \pm 0.038$ & $-0.02 \pm 0.002$ & $-0.173 \pm 0.026$ & $-0.009 \pm 0.001$ \\
\hline NGC 7591 & $-0.154 \pm 0.008$ & $-0.024 \pm 0.001$ & $-0.096 \pm 0.009$ & $-0.015 \pm 0.001$ \\
\hline IC 5309 & $-0.046 \pm 0.013$ & $-0.013 \pm 0.003$ & $-0.019 \pm 0.007$ & $-0.005 \pm 0.002$ \\
\hline NGC 7611 & $0.04 \pm 0.009$ & $0.014 \pm 0.003$ & $0.034 \pm 0.006$ & $0.012 \pm 0.002$ \\
\hline NGC 7623 & $-0.318 \pm 0.045$ & $-0.061 \pm 0.009$ & $-0.174 \pm 0.03$ & $-0.033 \pm 0.006$ \\
\hline NGC 7631 & $-0.128 \pm 0.007$ & $-0.033 \pm 0.002$ & $-0.007 \pm 0.004$ & $-0.002 \pm 0.001$ \\
\hline
\end{tabular}


A\&A 604, A4 (2017)

Table B.1. continued.

\begin{tabular}{|c|c|c|c|c|}
\hline $\begin{array}{l}\text { Name } \\
\text { (1) } \\
\end{array}$ & $\begin{array}{r}\nabla \text { lo } \\
\left(\operatorname{dex} / h_{\text {in }}\right) \\
(2) \\
\end{array}$ & $\begin{array}{r}\text { e) })_{\mathrm{L}, \mathrm{in}} \\
(\mathrm{dex} / \mathrm{kpc}) \\
(3) \\
\end{array}$ & $\begin{array}{r}\nabla \operatorname{lo} \\
\left(\operatorname{dex} / h_{\text {in }}\right) \\
(4) \\
\end{array}$ & $\begin{array}{r})_{\mathrm{M}, \mathrm{in}} \\
\quad(\mathrm{dex} / \mathrm{kpc}) \\
(5) \\
\end{array}$ \\
\hline NGC 7653 & $-0.178 \pm 0.012$ & $-0.047 \pm 0.003$ & $-0.14 \pm 0.009$ & $-0.037 \pm 0.002$ \\
\hline NGC 7671 & $-0.1 \pm 0.006$ & $-0.035 \pm 0.002$ & $-0.063 \pm 0.004$ & $-0.022 \pm 0.001$ \\
\hline NGC 7691 & $-0.324 \pm 0.026$ & $-0.065 \pm 0.005$ & $-0.103 \pm 0.013$ & $-0.021 \pm 0.003$ \\
\hline NGC 7716 & $-0.348 \pm 0.01$ & $-0.077 \pm 0.002$ & $-0.051 \pm 0.005$ & $-0.011 \pm 0.001$ \\
\hline NGC 7722 & $-0.002 \pm 0.016$ & $-0.001 \pm 0.005$ & $-0.078 \pm 0.009$ & $-0.027 \pm 0.003$ \\
\hline NGC 7738 & $-0.216 \pm 0.007$ & $-0.045 \pm 0.001$ & $-0.015 \pm 0.005$ & $-0.003 \pm 0.001$ \\
\hline UGC 12810 & $-0.369 \pm 0.01$ & $-0.044 \pm 0.001$ & $-0.117 \pm 0.007$ & $-0.014 \pm 0.001$ \\
\hline UGC 12816 & $-0.26 \pm 0.05$ & $-0.022 \pm 0.004$ & $-0.054 \pm 0.023$ & $-0.005 \pm 0.002$ \\
\hline NGC 7782 & $-0.423 \pm 0.009$ & $-0.064 \pm 0.001$ & $-0.093 \pm 0.006$ & $-0.014 \pm 0.001$ \\
\hline NGC 7787 & $-0.404 \pm 0.012$ & $-0.084 \pm 0.002$ & $-0.069 \pm 0.007$ & $-0.014 \pm 0.001$ \\
\hline UGC 12864 & $-0.155 \pm 0.014$ & $-0.013 \pm 0.001$ & $-0.119 \pm 0.008$ & $-0.01 \pm 0.001$ \\
\hline UGC 04455 & $-0.493 \pm 0.027$ & $-0.146 \pm 0.008$ & $-0.05 \pm 0.02$ & $-0.015 \pm 0.006$ \\
\hline UGC 06249 & $-0.509 \pm 0.051$ & $-0.045 \pm 0.005$ & $-0.042 \pm 0.031$ & $-0.004 \pm 0.003$ \\
\hline SN2002ji & $-0.252 \pm 0.009$ & $-0.26 \pm 0.009$ & $-0.144 \pm 0.009$ & $-0.149 \pm 0.009$ \\
\hline UGC 07129 & $0.048 \pm 0.025$ & $0.054 \pm 0.028$ & $0.076 \pm 0.025$ & $0.085 \pm 0.027$ \\
\hline UGC 08909 & $-0.18 \pm 0.021$ & $-0.214 \pm 0.025$ & $-0.117 \pm 0.01$ & $-0.139 \pm 0.011$ \\
\hline NGC 0495 & $-0.12 \pm 0.005$ & $-0.094 \pm 0.004$ & $-0.074 \pm 0.004$ & $-0.057 \pm 0.003$ \\
\hline KUG1349+143 & $0.035 \pm 0.017$ & $0.005 \pm 0.002$ & $-0.041 \pm 0.011$ & $-0.006 \pm 0.002$ \\
\hline CGCG163-062 & $0.144 \pm 0.025$ & $0.038 \pm 0.007$ & $0.035 \pm 0.015$ & $0.009 \pm 0.004$ \\
\hline NGC 5794 & $-0.048 \pm 0.008$ & $-0.013 \pm 0.002$ & $-0.002 \pm 0.005$ & $-0.0 \pm 0.001$ \\
\hline IC 1078 & $-0.523 \pm 0.017$ & $-0.108 \pm 0.003$ & $-0.027 \pm 0.01$ & $-0.006 \pm 0.002$ \\
\hline NGC 6977 & $-0.318 \pm 0.009$ & $-0.044 \pm 0.001$ & $-0.099 \pm 0.006$ & $-0.014 \pm 0.001$ \\
\hline SDSSJ015424 & $-0.028 \pm 0.014$ & $-0.01 \pm 0.005$ & $-0.013 \pm 0.015$ & $-0.005 \pm 0.005$ \\
\hline NGC 2691 & $0.245 \pm 0.026$ & $0.082 \pm 0.009$ & $0.109 \pm 0.021$ & $0.037 \pm 0.007$ \\
\hline NGC 2780 & $-0.163 \pm 0.027$ & $-0.014 \pm 0.002$ & $-0.1 \pm 0.017$ & $-0.008 \pm 0.001$ \\
\hline UGC 06517 & $-0.28 \pm 0.014$ & $-0.14 \pm 0.007$ & $-0.112 \pm 0.007$ & $-0.056 \pm 0.004$ \\
\hline NGC 5145 & $0.716 \pm 0.11$ & $0.271 \pm 0.042$ & $0.593 \pm 0.163$ & $0.224 \pm 0.062$ \\
\hline NGC 5950 & $-0.228 \pm 0.034$ & $-0.168 \pm 0.025$ & $-0.063 \pm 0.013$ & $-0.047 \pm 0.009$ \\
\hline UGC 10803 & $-0.095 \pm 0.005$ & $-0.073 \pm 0.004$ & $-0.003 \pm 0.003$ & $-0.003 \pm 0.002$ \\
\hline MCG-01-52-012 & $-0.132 \pm 0.011$ & $-0.189 \pm 0.015$ & $-0.122 \pm 0.008$ & $-0.175 \pm 0.011$ \\
\hline UGC 09837 & $-0.126 \pm 0.03$ & $-0.027 \pm 0.006$ & $-0.147 \pm 0.017$ & $-0.031 \pm 0.004$ \\
\hline UGC 12250 & $-1.324 \pm 0.068$ & $-0.089 \pm 0.005$ & $-0.296 \pm 0.053$ & $-0.02 \pm 0.004$ \\
\hline NGC 5947 & $-0.433 \pm 0.016$ & $-0.063 \pm 0.002$ & $-0.085 \pm 0.012$ & $-0.012 \pm 0.002$ \\
\hline
\end{tabular}


T. Ruiz-Lara et al.: Observational hints of radial migration in disc galaxies from CALIFA

Table B.2. Stellar metallicity inner gradients from the stellar content analysis (see Sect. 4).

\begin{tabular}{|c|c|c|c|c|}
\hline $\begin{array}{l}\text { Name } \\
\text { (1) }\end{array}$ & $\begin{array}{r}\nabla[1 \\
\left(\operatorname{dex} / h_{\text {in }}\right) \\
(2)\end{array}$ & $\begin{array}{r}(\mathrm{dex} / \mathrm{kpc}) \\
(3)\end{array}$ & $\begin{array}{r}\nabla[\mathrm{N} \\
\left(\operatorname{dex} / h_{\mathrm{in}}\right) \\
(4)\end{array}$ & $\begin{array}{r}(\mathrm{dex} / \mathrm{kpc}) \\
(5)\end{array}$ \\
\hline UGC 00005 & $-0.176 \pm 0.009$ & $-0.028 \pm 0.001$ & $0.007 \pm 0.011$ & $0.001 \pm 0.002$ \\
\hline NGC 7819 & $-0.124 \pm 0.027$ & $-0.008 \pm 0.002$ & $-0.752 \pm 0.037$ & $-0.049 \pm 0.002$ \\
\hline IC 1528 & $-0.017 \pm 0.009$ & $-0.004 \pm 0.002$ & $-0.15 \pm 0.012$ & $-0.036 \pm 0.003$ \\
\hline UGC 00036 & $-0.043 \pm 0.006$ & $-0.01 \pm 0.001$ & $0.046 \pm 0.007$ & $0.011 \pm 0.002$ \\
\hline NGC 0001 & $-0.071 \pm 0.01$ & $-0.016 \pm 0.002$ & $0.018 \pm 0.013$ & $0.004 \pm 0.003$ \\
\hline NGC 0023 & $1.887 \pm 0.105$ & $0.055 \pm 0.003$ & $0.026 \pm 0.121$ & $0.001 \pm 0.004$ \\
\hline NGC 0036 & $-0.077 \pm 0.011$ & $-0.008 \pm 0.001$ & $0.101 \pm 0.014$ & $0.011 \pm 0.001$ \\
\hline UGC 00139 & $-0.02 \pm 0.033$ & $-0.006 \pm 0.01$ & $-0.525 \pm 0.05$ & $-0.161 \pm 0.015$ \\
\hline MCG-02-02-030 & $-0.507 \pm 0.019$ & $-0.112 \pm 0.004$ & $-0.107 \pm 0.11$ & $-0.024 \pm 0.024$ \\
\hline UGC 00312 & $-0.097 \pm 0.014$ & $-0.017 \pm 0.003$ & $-0.033 \pm 0.03$ & $-0.006 \pm 0.005$ \\
\hline ESO540-G003 & $-0.727 \pm 0.048$ & $-0.215 \pm 0.014$ & $-0.711 \pm 0.117$ & $-0.21 \pm 0.034$ \\
\hline NGC 0160 & $-0.598 \pm 0.044$ & $-0.035 \pm 0.003$ & $-0.292 \pm 0.049$ & $-0.017 \pm 0.003$ \\
\hline NGC 0165 & $0.074 \pm 0.015$ & $0.013 \pm 0.003$ & $-0.137 \pm 0.02$ & $-0.024 \pm 0.004$ \\
\hline NGC 0171 & $-2.367 \pm 0.038$ & $-0.116 \pm 0.002$ & $-0.296 \pm 0.07$ & $-0.015 \pm 0.003$ \\
\hline NGC 0180 & $-0.337 \pm 0.006$ & $-0.04 \pm 0.001$ & $-0.079 \pm 0.013$ & $-0.009 \pm 0.002$ \\
\hline NGC 0214 & $-0.402 \pm 0.014$ & $-0.094 \pm 0.003$ & $-0.439 \pm 0.025$ & $-0.103 \pm 0.006$ \\
\hline NGC 0237 & $-0.079 \pm 0.008$ & $-0.029 \pm 0.003$ & $-0.21 \pm 0.021$ & $-0.078 \pm 0.008$ \\
\hline NGC 0234 & $0.062 \pm 0.017$ & $0.011 \pm 0.003$ & $0.056 \pm 0.022$ & $0.01 \pm 0.004$ \\
\hline NGC 0257 & $-0.27 \pm 0.009$ & $-0.049 \pm 0.002$ & $-0.271 \pm 0.013$ & $-0.049 \pm 0.002$ \\
\hline NGC 0309 & $-0.443 \pm 0.012$ & $-0.048 \pm 0.001$ & $-0.544 \pm 0.022$ & $-0.059 \pm 0.002$ \\
\hline NGC 0447 & $-0.58 \pm 0.027$ & $-0.057 \pm 0.003$ & $-0.35 \pm 0.026$ & $-0.035 \pm 0.003$ \\
\hline NGC 0477 & $-0.204 \pm 0.011$ & $-0.03 \pm 0.002$ & $-0.139 \pm 0.017$ & $-0.021 \pm 0.002$ \\
\hline IC 1683 & $-0.038 \pm 0.009$ & $-0.008 \pm 0.002$ & $-0.179 \pm 0.01$ & $-0.037 \pm 0.002$ \\
\hline NGC 0496 & $-0.323 \pm 0.01$ & $-0.059 \pm 0.002$ & $-0.516 \pm 0.015$ & $-0.093 \pm 0.003$ \\
\hline NGC 0528 & $-0.076 \pm 0.004$ & $-0.026 \pm 0.001$ & $-0.054 \pm 0.004$ & $-0.018 \pm 0.001$ \\
\hline NGC 0551 & $-0.214 \pm 0.005$ & $-0.042 \pm 0.001$ & $-0.22 \pm 0.009$ & $-0.043 \pm 0.002$ \\
\hline NGC 0570 & $-0.037 \pm 0.01$ & $-0.007 \pm 0.002$ & $-0.054 \pm 0.018$ & $-0.01 \pm 0.003$ \\
\hline UGC 01271 & $0.005 \pm 0.006$ & $0.001 \pm 0.001$ & $0.006 \pm 0.007$ & $0.002 \pm 0.002$ \\
\hline NGC 0716 & $-0.161 \pm 0.018$ & $-0.048 \pm 0.005$ & $-0.24 \pm 0.028$ & $-0.071 \pm 0.008$ \\
\hline NGC 0768 & $-0.262 \pm 0.011$ & $-0.04 \pm 0.002$ & $-0.076 \pm 0.015$ & $-0.012 \pm 0.002$ \\
\hline NGC 0776 & $-0.167 \pm 0.011$ & $-0.029 \pm 0.002$ & $-0.008 \pm 0.013$ & $-0.001 \pm 0.002$ \\
\hline NGC 0787 & $-0.215 \pm 0.014$ & $-0.053 \pm 0.003$ & $-0.11 \pm 0.014$ & $-0.027 \pm 0.003$ \\
\hline NGC 0842 & $-0.109 \pm 0.005$ & $-0.025 \pm 0.001$ & $-0.143 \pm 0.005$ & $-0.033 \pm 0.001$ \\
\hline UGC 01659 & $-1.063 \pm 0.114$ & $-0.036 \pm 0.004$ & $-0.061 \pm 0.129$ & $-0.002 \pm 0.004$ \\
\hline NGC 0873 & $0.118 \pm 0.014$ & $0.042 \pm 0.005$ & $0.099 \pm 0.023$ & $0.036 \pm 0.008$ \\
\hline NGC 0924 & $0.054 \pm 0.039$ & $0.01 \pm 0.007$ & $-0.052 \pm 0.052$ & $-0.01 \pm 0.009$ \\
\hline UGC 01918 & $-0.221 \pm 0.046$ & $-0.035 \pm 0.007$ & $0.005 \pm 0.055$ & $0.001 \pm 0.009$ \\
\hline NGC 0932 & $-0.059 \pm 0.008$ & $-0.011 \pm 0.002$ & $-0.006 \pm 0.009$ & $-0.001 \pm 0.002$ \\
\hline NGC 0941 & $0.146 \pm 0.01$ & $0.065 \pm 0.005$ & $0.116 \pm 0.02$ & $0.052 \pm 0.009$ \\
\hline NGC 0976 & $-0.408 \pm 0.095$ & $-0.124 \pm 0.029$ & $-0.174 \pm 0.139$ & $-0.053 \pm 0.042$ \\
\hline NGC 0991 & $0.011 \pm 0.015$ & $0.004 \pm 0.005$ & $0.185 \pm 0.024$ & $0.064 \pm 0.008$ \\
\hline UGC 02099 & $-0.206 \pm 0.019$ & $-0.02 \pm 0.002$ & $-0.025 \pm 0.023$ & $-0.002 \pm 0.002$ \\
\hline UGC 02134 & $0.115 \pm 0.082$ & $0.005 \pm 0.004$ & $1.15 \pm 0.181$ & $0.054 \pm 0.009$ \\
\hline NGC 1070 & $-0.073 \pm 0.009$ & $-0.031 \pm 0.004$ & $0.032 \pm 0.016$ & $0.013 \pm 0.007$ \\
\hline NGC 1094 & $0.208 \pm 0.018$ & $0.076 \pm 0.007$ & $-0.043 \pm 0.02$ & $-0.016 \pm 0.007$ \\
\hline NGC 1093 & $-0.159 \pm 0.009$ & $-0.035 \pm 0.002$ & $0.007 \pm 0.012$ & $0.002 \pm 0.003$ \\
\hline UGC 02311 & $-0.255 \pm 0.01$ & $-0.045 \pm 0.002$ & $-0.353 \pm 0.013$ & $-0.062 \pm 0.002$ \\
\hline UGC 02403 & $-0.455 \pm 0.024$ & $-0.092 \pm 0.005$ & $-0.92 \pm 0.02$ & $-0.186 \pm 0.004$ \\
\hline UGC 02443 & $0.343 \pm 0.03$ & $0.093 \pm 0.008$ & $-0.292 \pm 0.082$ & $-0.079 \pm 0.022$ \\
\hline NGC 1167 & $0.038 \pm 0.011$ & $0.004 \pm 0.001$ & $-0.102 \pm 0.013$ & $-0.012 \pm 0.002$ \\
\hline NGC 1211 & $0.355 \pm 0.056$ & $0.055 \pm 0.009$ & $0.528 \pm 0.063$ & $0.081 \pm 0.01$ \\
\hline UGC 02690 & $-0.156 \pm 0.021$ & $-0.032 \pm 0.004$ & $-0.783 \pm 0.024$ & $-0.162 \pm 0.005$ \\
\hline MCG-01-10-019 & $-0.04 \pm 0.015$ & $-0.006 \pm 0.002$ & $-0.522 \pm 0.017$ & $-0.074 \pm 0.002$ \\
\hline NGC 1645 & $-0.002 \pm 0.011$ & $-0.0 \pm 0.002$ & $-0.07 \pm 0.013$ & $-0.013 \pm 0.002$ \\
\hline NGC 1659 & $-0.085 \pm 0.008$ & $-0.023 \pm 0.002$ & $-0.081 \pm 0.023$ & $-0.022 \pm 0.006$ \\
\hline NGC 1665 & $0.001 \pm 0.007$ & $0.0 \pm 0.002$ & $-0.051 \pm 0.006$ & $-0.015 \pm 0.002$ \\
\hline NGC 1666 & $-0.189 \pm 0.009$ & $-0.074 \pm 0.003$ & $-0.142 \pm 0.01$ & $-0.056 \pm 0.004$ \\
\hline NGC 1667 & $-0.012 \pm 0.006$ & $-0.003 \pm 0.002$ & $0.028 \pm 0.009$ & $0.008 \pm 0.003$ \\
\hline UGC 03253 & $-0.095 \pm 0.018$ & $-0.026 \pm 0.005$ & $0.089 \pm 0.023$ & $0.025 \pm 0.006$ \\
\hline NGC 2253 & $-0.059 \pm 0.007$ & $-0.02 \pm 0.002$ & $0.133 \pm 0.008$ & $0.046 \pm 0.003$ \\
\hline NGC 2347 & $-0.258 \pm 0.008$ & $-0.055 \pm 0.002$ & $-0.224 \pm 0.013$ & $-0.048 \pm 0.003$ \\
\hline
\end{tabular}

Notes. This table shows the gradients for the light- and mass-weighted $[\mathrm{M} / \mathrm{H}]\left(\mathrm{dex} / h_{\text {in }}\right.$ and $\left.\mathrm{dex} / \mathrm{kpc}\right)$ represented in Fig. 6. 
A\&A 604, A4 (2017)

Table B.2. continued.

\begin{tabular}{|c|c|c|c|c|}
\hline \multirow{2}{*}{$\begin{array}{l}\text { Name } \\
\text { (1) }\end{array}$} & \multicolumn{2}{|c|}{$\nabla[\mathrm{M} / \mathrm{H}]_{\mathrm{L}, \text { in }}$} & \multicolumn{2}{|c|}{$\nabla[\mathrm{M} / \mathrm{H}]_{\mathrm{M}, \text { in }}$} \\
\hline & $\begin{array}{r}\left(\operatorname{dex} / h_{\text {in }}\right) \\
(2)\end{array}$ & $\begin{array}{r}(\mathrm{dex} / \mathrm{kpc}) \\
(3)\end{array}$ & $\begin{array}{r}\left(\operatorname{dex} / h_{\text {in }}\right) \\
(4)\end{array}$ & $\begin{array}{r}(\mathrm{dex} / \mathrm{kpc}) \\
(5)\end{array}$ \\
\hline UGC 03944 & $-0.119 \pm 0.011$ & $-0.038 \pm 0.003$ & $-0.318 \pm 0.03$ & $-0.101 \pm 0.009$ \\
\hline UGC 03973 & $0.221 \pm 0.023$ & $0.029 \pm 0.003$ & $-0.091 \pm 0.027$ & $-0.012 \pm 0.003$ \\
\hline UGC 03995 & $-0.232 \pm 0.008$ & $-0.031 \pm 0.001$ & $-0.075 \pm 0.01$ & $-0.01 \pm 0.001$ \\
\hline NGC 2449 & $-0.055 \pm 0.005$ & $-0.012 \pm 0.001$ & $-0.026 \pm 0.008$ & $-0.006 \pm 0.002$ \\
\hline NGC 2487 & $0.116 \pm 0.015$ & $0.016 \pm 0.002$ & $0.24 \pm 0.021$ & $0.032 \pm 0.003$ \\
\hline UGC 04195 & $-0.158 \pm 0.024$ & $-0.023 \pm 0.004$ & $-0.172 \pm 0.026$ & $-0.025 \pm 0.004$ \\
\hline NGC 2530 & $0.105 \pm 0.01$ & $0.023 \pm 0.002$ & $-0.34 \pm 0.018$ & $-0.073 \pm 0.004$ \\
\hline NGC 2540 & $-0.114 \pm 0.015$ & $-0.021 \pm 0.003$ & $-0.199 \pm 0.016$ & $-0.037 \pm 0.003$ \\
\hline NGC 2543 & $-0.108 \pm 0.014$ & $-0.028 \pm 0.004$ & $0.103 \pm 0.017$ & $0.027 \pm 0.004$ \\
\hline UGC 04308 & $-0.191 \pm 0.01$ & $-0.045 \pm 0.002$ & $-0.377 \pm 0.025$ & $-0.09 \pm 0.006$ \\
\hline NGC 2553 & $0.072 \pm 0.006$ & $0.017 \pm 0.001$ & $0.119 \pm 0.005$ & $0.027 \pm 0.001$ \\
\hline UGC 04262 & $-0.203 \pm 0.014$ & $-0.035 \pm 0.002$ & $-0.295 \pm 0.018$ & $-0.051 \pm 0.003$ \\
\hline NGC 2558 & $-0.151 \pm 0.011$ & $-0.027 \pm 0.002$ & $-0.016 \pm 0.023$ & $-0.003 \pm 0.004$ \\
\hline NGC 2565 & $-0.156 \pm 0.006$ & $-0.044 \pm 0.002$ & $-0.042 \pm 0.01$ & $-0.012 \pm 0.003$ \\
\hline NGC 2572 & $-0.194 \pm 0.008$ & $-0.04 \pm 0.002$ & $-0.197 \pm 0.019$ & $-0.041 \pm 0.004$ \\
\hline UGC 04375 & $-0.135 \pm 0.006$ & $-0.05 \pm 0.002$ & $-0.087 \pm 0.009$ & $-0.032 \pm 0.003$ \\
\hline NGC 2596 & $-0.058 \pm 0.034$ & $-0.007 \pm 0.004$ & $0.07 \pm 0.026$ & $0.008 \pm 0.003$ \\
\hline NGC 2595 & $-0.164 \pm 0.014$ & $-0.019 \pm 0.002$ & $-0.135 \pm 0.029$ & $-0.016 \pm 0.003$ \\
\hline NGC 2604 & $-0.135 \pm 0.017$ & $-0.046 \pm 0.006$ & $-0.217 \pm 0.034$ & $-0.073 \pm 0.012$ \\
\hline NGC 2639 & $-0.073 \pm 0.003$ & $-0.026 \pm 0.001$ & $-0.065 \pm 0.005$ & $-0.023 \pm 0.002$ \\
\hline NGC 2730 & $0.151 \pm 0.013$ & $0.024 \pm 0.002$ & $0.341 \pm 0.032$ & $0.054 \pm 0.005$ \\
\hline NGC 2805 & $1.563 \pm 0.443$ & $0.228 \pm 0.065$ & $5.439 \pm 0.845$ & $0.793 \pm 0.123$ \\
\hline NGC 2906 & $-0.144 \pm 0.003$ & $-0.072 \pm 0.002$ & $-0.062 \pm 0.005$ & $-0.031 \pm 0.003$ \\
\hline NGC 2916 & $-0.363 \pm 0.016$ & $-0.064 \pm 0.003$ & $0.001 \pm 0.027$ & $0.0 \pm 0.005$ \\
\hline UGC 05108 & $-2.359 \pm 0.049$ & $-0.06 \pm 0.001$ & $-0.545 \pm 0.052$ & $-0.014 \pm 0.001$ \\
\hline UGC 05359 & $-0.226 \pm 0.011$ & $-0.035 \pm 0.002$ & $-0.056 \pm 0.014$ & $-0.009 \pm 0.002$ \\
\hline UGC 05396 & $-0.193 \pm 0.012$ & $-0.03 \pm 0.002$ & $-0.017 \pm 0.03$ & $-0.003 \pm 0.005$ \\
\hline NGC 3106 & $-0.069 \pm 0.01$ & $-0.009 \pm 0.001$ & $0.12 \pm 0.019$ & $0.016 \pm 0.002$ \\
\hline NGC 3300 & $-0.085 \pm 0.005$ & $-0.03 \pm 0.002$ & $-0.092 \pm 0.005$ & $-0.033 \pm 0.002$ \\
\hline NGC 3381 & $-0.095 \pm 0.008$ & $-0.046 \pm 0.004$ & $-0.058 \pm 0.018$ & $-0.028 \pm 0.009$ \\
\hline IC 0674 & $-0.217 \pm 0.006$ & $-0.035 \pm 0.001$ & $0.035 \pm 0.005$ & $0.006 \pm 0.001$ \\
\hline UGC 06312 & $0.034 \pm 0.011$ & $0.005 \pm 0.002$ & $-0.022 \pm 0.018$ & $-0.003 \pm 0.003$ \\
\hline NGC 3614 & $-0.637 \pm 0.023$ & $-0.132 \pm 0.005$ & $-0.562 \pm 0.038$ & $-0.116 \pm 0.008$ \\
\hline NGC 3687 & $0.023 \pm 0.016$ & $0.01 \pm 0.007$ & $0.136 \pm 0.021$ & $0.06 \pm 0.009$ \\
\hline NGC 3811 & $-0.147 \pm 0.007$ & $-0.057 \pm 0.003$ & $-0.084 \pm 0.028$ & $-0.032 \pm 0.011$ \\
\hline NGC 3815 & $-0.096 \pm 0.007$ & $-0.034 \pm 0.002$ & $-0.086 \pm 0.011$ & $-0.03 \pm 0.004$ \\
\hline NGC 3994 & $-0.091 \pm 0.005$ & $-0.071 \pm 0.004$ & $-0.003 \pm 0.008$ & $-0.003 \pm 0.006$ \\
\hline NGC 4003 & $0.017 \pm 0.007$ & $0.003 \pm 0.001$ & $-0.16 \pm 0.01$ & $-0.024 \pm 0.002$ \\
\hline UGC 07012 & $-0.01 \pm 0.017$ & $-0.006 \pm 0.01$ & $0.386 \pm 0.034$ & $0.22 \pm 0.02$ \\
\hline NGC 4047 & $-0.158 \pm 0.012$ & $-0.064 \pm 0.005$ & $-0.01 \pm 0.016$ & $-0.004 \pm 0.006$ \\
\hline UGC 07145 & $-0.56 \pm 0.011$ & $-0.088 \pm 0.002$ & $-1.04 \pm 0.02$ & $-0.164 \pm 0.003$ \\
\hline NGC 4185 & $-0.16 \pm 0.012$ & $-0.022 \pm 0.002$ & $0.018 \pm 0.014$ & $0.002 \pm 0.002$ \\
\hline NGC 4210 & $0.078 \pm 0.018$ & $0.014 \pm 0.003$ & $0.441 \pm 0.031$ & $0.077 \pm 0.005$ \\
\hline NGC 4470 & $0.042 \pm 0.005$ & $0.024 \pm 0.003$ & $-0.32 \pm 0.006$ & $-0.181 \pm 0.003$ \\
\hline NGC 4711 & $-0.2 \pm 0.012$ & $-0.051 \pm 0.003$ & $-0.022 \pm 0.029$ & $-0.006 \pm 0.007$ \\
\hline UGC 08004 & $-0.151 \pm 0.015$ & $-0.022 \pm 0.002$ & $-0.522 \pm 0.023$ & $-0.077 \pm 0.003$ \\
\hline NGC 4961 & $0.656 \pm 0.019$ & $0.256 \pm 0.008$ & $0.502 \pm 0.035$ & $0.196 \pm 0.014$ \\
\hline UGC 08231 & $0.13 \pm 0.007$ & $0.057 \pm 0.003$ & $0.357 \pm 0.012$ & $0.158 \pm 0.005$ \\
\hline NGC 5000 & $0.021 \pm 0.011$ & $0.004 \pm 0.002$ & $-0.059 \pm 0.017$ & $-0.011 \pm 0.003$ \\
\hline NGC 5016 & $0.009 \pm 0.007$ & $0.004 \pm 0.004$ & $-0.076 \pm 0.026$ & $-0.038 \pm 0.013$ \\
\hline NGC 5157 & $-0.29 \pm 0.006$ & $-0.04 \pm 0.001$ & $0.066 \pm 0.007$ & $0.009 \pm 0.001$ \\
\hline NGC 5205 & $-0.178 \pm 0.01$ & $-0.1 \pm 0.006$ & $-0.143 \pm 0.026$ & $-0.081 \pm 0.015$ \\
\hline NGC 5267 & $-0.605 \pm 0.014$ & $-0.062 \pm 0.001$ & $0.02 \pm 0.019$ & $0.002 \pm 0.002$ \\
\hline NGC 5320 & $-0.227 \pm 0.007$ & $-0.06 \pm 0.002$ & $0.058 \pm 0.013$ & $0.016 \pm 0.003$ \\
\hline UGC 08781 & $-0.191 \pm 0.01$ & $-0.025 \pm 0.001$ & $-0.164 \pm 0.013$ & $-0.021 \pm 0.002$ \\
\hline NGC 5376 & $-0.244 \pm 0.011$ & $-0.084 \pm 0.004$ & $0.145 \pm 0.01$ & $0.05 \pm 0.004$ \\
\hline NGC 5379 & $0.107 \pm 0.006$ & $0.059 \pm 0.003$ & $0.041 \pm 0.008$ & $0.023 \pm 0.004$ \\
\hline NGC 5378 & $-0.091 \pm 0.013$ & $-0.018 \pm 0.003$ & $0.18 \pm 0.016$ & $0.035 \pm 0.003$ \\
\hline NGC 5406 & $-0.786 \pm 0.069$ & $-0.036 \pm 0.003$ & $-0.231 \pm 0.048$ & $-0.01 \pm 0.002$ \\
\hline NGC 5473 & $-0.181 \pm 0.006$ & $-0.077 \pm 0.002$ & $-0.181 \pm 0.007$ & $-0.077 \pm 0.003$ \\
\hline NGC 5480 & $-0.101 \pm 0.037$ & $-0.034 \pm 0.013$ & $0.492 \pm 0.07$ & $0.167 \pm 0.024$ \\
\hline UGC 09067 & $-0.055 \pm 0.009$ & $-0.012 \pm 0.002$ & $-0.048 \pm 0.015$ & $-0.01 \pm 0.003$ \\
\hline NGC 5520 & $-0.05 \pm 0.007$ & $-0.03 \pm 0.004$ & $-0.097 \pm 0.017$ & $-0.059 \pm 0.011$ \\
\hline NGC 5633 & $-0.064 \pm 0.005$ & $-0.045 \pm 0.003$ & $0.05 \pm 0.01$ & $0.036 \pm 0.007$ \\
\hline
\end{tabular}


T. Ruiz-Lara et al.: Observational hints of radial migration in disc galaxies from CALIFA

Table B.2. continued.

\begin{tabular}{|c|c|c|c|c|}
\hline \multirow{3}{*}{$\begin{array}{l}\text { Name } \\
\text { (1) }\end{array}$} & \multicolumn{2}{|c|}{$\nabla[\mathrm{M} / \mathrm{H}]_{\mathrm{L}, \text { in }}$} & \multicolumn{2}{|c|}{$\nabla[\mathrm{M} / \mathrm{H}]_{\mathrm{M}, \text { in }}$} \\
\hline & $\left(\operatorname{dex} / h_{\text {in }}\right)$ & $(\mathrm{dex} / \mathrm{kpc})$ & $\left(\mathrm{dex} / h_{\mathrm{in}}\right)$ & $(\mathrm{dex} / \mathrm{kpc})$ \\
\hline & $(2)$ & (3) & $(4)$ & (5) \\
\hline NGC 5657 & $-0.154 \pm 0.007$ & $-0.039 \pm 0.002$ & $-0.046 \pm 0.013$ & $-0.012 \pm 0.003$ \\
\hline NGC 5720 & $-0.343 \pm 0.005$ & $-0.05 \pm 0.001$ & $-0.397 \pm 0.01$ & $-0.058 \pm 0.001$ \\
\hline NGC 5732 & $0.131 \pm 0.012$ & $0.051 \pm 0.005$ & $0.01 \pm 0.023$ & $0.004 \pm 0.009$ \\
\hline UGC 09476 & $0.017 \pm 0.008$ & $0.004 \pm 0.002$ & $-0.033 \pm 0.02$ & $-0.008 \pm 0.005$ \\
\hline NGC 5784 & $-0.046 \pm 0.009$ & $-0.007 \pm 0.001$ & $-0.114 \pm 0.013$ & $-0.017 \pm 0.002$ \\
\hline UGC 09598 & $-0.215 \pm 0.009$ & $-0.037 \pm 0.001$ & $-0.26 \pm 0.018$ & $-0.044 \pm 0.003$ \\
\hline NGC 5876 & $-0.279 \pm 0.011$ & $-0.039 \pm 0.002$ & $0.033 \pm 0.015$ & $0.005 \pm 0.002$ \\
\hline NGC 5888 & $0.002 \pm 0.011$ & $0.0 \pm 0.001$ & $0.028 \pm 0.008$ & $0.003 \pm 0.001$ \\
\hline UGC 09777 & $-0.092 \pm 0.009$ & $-0.037 \pm 0.003$ & $-0.193 \pm 0.013$ & $-0.077 \pm 0.005$ \\
\hline UGC 09842 & $-0.129 \pm 0.013$ & $-0.019 \pm 0.002$ & $-0.275 \pm 0.023$ & $-0.041 \pm 0.003$ \\
\hline NGC 5957 & $-0.113 \pm 0.006$ & $-0.045 \pm 0.002$ & $0.137 \pm 0.009$ & $0.054 \pm 0.004$ \\
\hline NGC 5971 & $-0.164 \pm 0.022$ & $-0.039 \pm 0.005$ & $-0.216 \pm 0.049$ & $-0.051 \pm 0.012$ \\
\hline IC 4566 & $-0.142 \pm 0.008$ & $-0.03 \pm 0.002$ & $-0.028 \pm 0.012$ & $-0.006 \pm 0.002$ \\
\hline NGC 5980 & $-0.025 \pm 0.007$ & $-0.007 \pm 0.002$ & $-0.059 \pm 0.009$ & $-0.018 \pm 0.003$ \\
\hline NGC 6004 & $-0.075 \pm 0.009$ & $-0.016 \pm 0.002$ & $0.067 \pm 0.009$ & $0.014 \pm 0.002$ \\
\hline IC 1151 & $0.109 \pm 0.007$ & $0.042 \pm 0.003$ & $0.038 \pm 0.014$ & $0.015 \pm 0.005$ \\
\hline NGC 6032 & $0.701 \pm 0.123$ & $0.024 \pm 0.004$ & $-1.68 \pm 0.312$ & $-0.057 \pm 0.011$ \\
\hline NGC 6060 & $-0.145 \pm 0.012$ & $-0.022 \pm 0.002$ & $-0.078 \pm 0.013$ & $-0.012 \pm 0.002$ \\
\hline NGC 6063 & $0.126 \pm 0.023$ & $0.03 \pm 0.006$ & $-0.094 \pm 0.047$ & $-0.022 \pm 0.011$ \\
\hline IC 1199 & $-0.297 \pm 0.017$ & $-0.051 \pm 0.003$ & $-0.238 \pm 0.026$ & $-0.041 \pm 0.005$ \\
\hline NGC 6154 & $-0.197 \pm 0.012$ & $-0.028 \pm 0.002$ & $0.22 \pm 0.013$ & $0.032 \pm 0.002$ \\
\hline NGC 6155 & $0.091 \pm 0.007$ & $0.046 \pm 0.004$ & $-0.081 \pm 0.011$ & $-0.042 \pm 0.005$ \\
\hline NGC 6186 & $0.039 \pm 0.026$ & $0.005 \pm 0.003$ & $0.412 \pm 0.045$ & $0.051 \pm 0.005$ \\
\hline NGC 6278 & $0.043 \pm 0.007$ & $0.009 \pm 0.001$ & $-0.043 \pm 0.007$ & $-0.009 \pm 0.002$ \\
\hline NGC 6301 & $-0.279 \pm 0.015$ & $-0.023 \pm 0.001$ & $-0.206 \pm 0.027$ & $-0.017 \pm 0.002$ \\
\hline NGC 6314 & $-0.029 \pm 0.01$ & $-0.004 \pm 0.001$ & $-0.037 \pm 0.015$ & $-0.005 \pm 0.002$ \\
\hline UGC 10796 & $-0.177 \pm 0.027$ & $-0.022 \pm 0.003$ & $-0.794 \pm 0.035$ & $-0.101 \pm 0.004$ \\
\hline UGC 10811 & $-0.342 \pm 0.008$ & $-0.048 \pm 0.001$ & $-0.031 \pm 0.014$ & $-0.004 \pm 0.002$ \\
\hline IC 1256 & $-0.0 \pm 0.011$ & $-0.0 \pm 0.003$ & $0.051 \pm 0.011$ & $0.014 \pm 0.003$ \\
\hline NGC 6394 & $-0.041 \pm 0.01$ & $-0.006 \pm 0.001$ & $-0.159 \pm 0.016$ & $-0.022 \pm 0.002$ \\
\hline UGC 10905 & $-0.036 \pm 0.011$ & $-0.003 \pm 0.001$ & $-0.048 \pm 0.014$ & $-0.004 \pm 0.001$ \\
\hline NGC 6427 & $-0.086 \pm 0.004$ & $-0.029 \pm 0.001$ & $-0.047 \pm 0.004$ & $-0.016 \pm 0.001$ \\
\hline NGC 6478 & $-0.465 \pm 0.022$ & $-0.049 \pm 0.002$ & $0.021 \pm 0.018$ & $0.002 \pm 0.002$ \\
\hline NGC 6497 & $-0.454 \pm 0.012$ & $-0.053 \pm 0.001$ & $0.117 \pm 0.023$ & $0.014 \pm 0.003$ \\
\hline UGC 11228 & $0.027 \pm 0.006$ & $0.005 \pm 0.001$ & $0.105 \pm 0.006$ & $0.02 \pm 0.001$ \\
\hline UGC 11262 & $-0.206 \pm 0.011$ & $-0.031 \pm 0.002$ & $-0.447 \pm 0.016$ & $-0.067 \pm 0.002$ \\
\hline MCG-02-51-004 & $-0.07 \pm 0.015$ & $-0.013 \pm 0.003$ & $-0.046 \pm 0.021$ & $-0.009 \pm 0.004$ \\
\hline NGC 6941 & $-0.369 \pm 0.009$ & $-0.049 \pm 0.001$ & $0.027 \pm 0.013$ & $0.004 \pm 0.002$ \\
\hline NGC 6945 & $-0.004 \pm 0.008$ & $-0.001 \pm 0.002$ & $-0.102 \pm 0.008$ & $-0.024 \pm 0.002$ \\
\hline NGC 6978 & $0.028 \pm 0.006$ & $0.005 \pm 0.001$ & $-0.074 \pm 0.009$ & $-0.013 \pm 0.002$ \\
\hline UGC 11649 & $-0.171 \pm 0.01$ & $-0.043 \pm 0.003$ & $-0.151 \pm 0.024$ & $-0.038 \pm 0.006$ \\
\hline NGC 7047 & $-0.431 \pm 0.015$ & $-0.063 \pm 0.002$ & $-0.042 \pm 0.017$ & $-0.006 \pm 0.002$ \\
\hline NGC 7311 & $-0.139 \pm 0.005$ & $-0.038 \pm 0.001$ & $-0.075 \pm 0.008$ & $-0.021 \pm 0.002$ \\
\hline NGC 7321 & $-0.236 \pm 0.008$ & $-0.043 \pm 0.001$ & $-0.168 \pm 0.009$ & $-0.031 \pm 0.002$ \\
\hline UGC 12185 & $0.134 \pm 0.02$ & $0.011 \pm 0.002$ & $-0.11 \pm 0.037$ & $-0.009 \pm 0.003$ \\
\hline UGC 12224 & $-0.408 \pm 0.02$ & $-0.088 \pm 0.004$ & $-0.456 \pm 0.035$ & $-0.099 \pm 0.008$ \\
\hline NGC 7466 & $-0.354 \pm 0.009$ & $-0.047 \pm 0.001$ & $-0.725 \pm 0.01$ & $-0.096 \pm 0.001$ \\
\hline NGC 7489 & $-0.317 \pm 0.007$ & $-0.058 \pm 0.001$ & $-0.518 \pm 0.01$ & $-0.095 \pm 0.002$ \\
\hline NGC 7536 & $0.676 \pm 0.069$ & $0.078 \pm 0.008$ & $0.225 \pm 0.064$ & $0.026 \pm 0.007$ \\
\hline NGC 7549 & $-0.124 \pm 0.016$ & $-0.015 \pm 0.002$ & $-0.009 \pm 0.021$ & $-0.001 \pm 0.003$ \\
\hline NGC 7563 & $0.066 \pm 0.025$ & $0.004 \pm 0.001$ & $0.045 \pm 0.023$ & $0.002 \pm 0.001$ \\
\hline NGC 7591 & $-0.169 \pm 0.01$ & $-0.026 \pm 0.002$ & $-0.283 \pm 0.018$ & $-0.044 \pm 0.003$ \\
\hline IC 5309 & $-0.046 \pm 0.014$ & $-0.013 \pm 0.004$ & $-0.265 \pm 0.018$ & $-0.072 \pm 0.005$ \\
\hline NGC 7611 & $-0.221 \pm 0.009$ & $-0.08 \pm 0.003$ & $-0.249 \pm 0.009$ & $-0.09 \pm 0.003$ \\
\hline NGC 7623 & $-0.155 \pm 0.038$ & $-0.03 \pm 0.007$ & $-0.159 \pm 0.031$ & $-0.031 \pm 0.006$ \\
\hline NGC 7631 & $-0.092 \pm 0.009$ & $-0.024 \pm 0.002$ & $-0.09 \pm 0.01$ & $-0.023 \pm 0.003$ \\
\hline NGC 7653 & $-0.001 \pm 0.012$ & $-0.0 \pm 0.003$ & $0.02 \pm 0.031$ & $0.005 \pm 0.008$ \\
\hline NGC 7671 & $-0.038 \pm 0.005$ & $-0.013 \pm 0.002$ & $-0.062 \pm 0.006$ & $-0.022 \pm 0.002$ \\
\hline NGC 7691 & $0.012 \pm 0.023$ & $0.002 \pm 0.005$ & $0.333 \pm 0.029$ & $0.067 \pm 0.006$ \\
\hline NGC 7716 & $-0.249 \pm 0.006$ & $-0.055 \pm 0.001$ & $-0.009 \pm 0.009$ & $-0.002 \pm 0.002$ \\
\hline NGC 7722 & $0.119 \pm 0.016$ & $0.041 \pm 0.005$ & $0.136 \pm 0.02$ & $0.047 \pm 0.007$ \\
\hline NGC 7738 & $-0.186 \pm 0.007$ & $-0.039 \pm 0.002$ & $0.023 \pm 0.011$ & $0.005 \pm 0.002$ \\
\hline UGC 12810 & $-0.158 \pm 0.012$ & $-0.019 \pm 0.001$ & $-0.332 \pm 0.016$ & $-0.04 \pm 0.002$ \\
\hline
\end{tabular}


A\&A 604, A4 (2017)

Table B.2. continued.

\begin{tabular}{|c|c|c|c|c|}
\hline $\begin{array}{l}\text { Name } \\
\text { (1) }\end{array}$ & $\begin{array}{r}\nabla[ \\
\left(\operatorname{dex} / h_{\text {in }}\right) \\
(2)\end{array}$ & $\begin{array}{r}(\mathrm{dex} / \mathrm{kpc}) \\
(3) \\
\end{array}$ & $\begin{array}{r}\nabla[\mathrm{\Lambda} \\
\left(\mathrm{dex} / h_{\mathrm{in}}\right) \\
(4) \\
\end{array}$ & $\begin{array}{r}(\mathrm{dex} / \mathrm{kpc}) \\
(5) \\
\end{array}$ \\
\hline UGC 12816 & $-0.381 \pm 0.037$ & $-0.033 \pm 0.003$ & $-0.744 \pm 0.052$ & $-0.064 \pm 0.004$ \\
\hline NGC 7782 & $-0.132 \pm 0.008$ & $-0.02 \pm 0.001$ & $-0.061 \pm 0.011$ & $-0.009 \pm 0.002$ \\
\hline NGC 7787 & $0.093 \pm 0.015$ & $0.019 \pm 0.003$ & $0.156 \pm 0.015$ & $0.033 \pm 0.003$ \\
\hline UGC 12864 & $-0.189 \pm 0.016$ & $-0.016 \pm 0.001$ & $-0.262 \pm 0.025$ & $-0.022 \pm 0.002$ \\
\hline UGC 04455 & $-0.095 \pm 0.013$ & $-0.028 \pm 0.004$ & $0.007 \pm 0.012$ & $0.002 \pm 0.004$ \\
\hline UGC 06249 & $-0.128 \pm 0.059$ & $-0.011 \pm 0.005$ & $-0.036 \pm 0.068$ & $-0.003 \pm 0.006$ \\
\hline SN2002ji & $0.013 \pm 0.01$ & $0.014 \pm 0.01$ & $0.223 \pm 0.039$ & $0.231 \pm 0.04$ \\
\hline UGC 07129 & $-0.331 \pm 0.024$ & $-0.37 \pm 0.026$ & $0.247 \pm 0.034$ & $0.276 \pm 0.037$ \\
\hline UGC 08909 & $-0.364 \pm 0.026$ & $-0.431 \pm 0.031$ & $-0.507 \pm 0.046$ & $-0.6 \pm 0.055$ \\
\hline NGC 0495 & $0.066 \pm 0.005$ & $0.051 \pm 0.004$ & $0.041 \pm 0.007$ & $0.032 \pm 0.006$ \\
\hline KUG1349+143 & $0.245 \pm 0.02$ & $0.034 \pm 0.003$ & $-0.143 \pm 0.022$ & $-0.02 \pm 0.003$ \\
\hline CGCG163-062 & $-0.385 \pm 0.029$ & $-0.102 \pm 0.008$ & $-0.52 \pm 0.03$ & $-0.138 \pm 0.008$ \\
\hline NGC 5794 & $-0.008 \pm 0.006$ & $-0.002 \pm 0.002$ & $-0.034 \pm 0.005$ & $-0.01 \pm 0.001$ \\
\hline IC 1078 & $-0.773 \pm 0.023$ & $-0.16 \pm 0.005$ & $-0.795 \pm 0.036$ & $-0.165 \pm 0.008$ \\
\hline NGC 6977 & $-0.174 \pm 0.012$ & $-0.024 \pm 0.002$ & $-0.054 \pm 0.014$ & $-0.007 \pm 0.002$ \\
\hline SDSSJ015424 & $0.024 \pm 0.012$ & $0.009 \pm 0.004$ & $-0.04 \pm 0.022$ & $-0.015 \pm 0.008$ \\
\hline NGC 2691 & $0.218 \pm 0.029$ & $0.073 \pm 0.01$ & $0.352 \pm 0.034$ & $0.118 \pm 0.011$ \\
\hline NGC 2780 & $0.282 \pm 0.031$ & $0.024 \pm 0.003$ & $0.281 \pm 0.047$ & $0.024 \pm 0.004$ \\
\hline UGC 06517 & $-0.152 \pm 0.013$ & $-0.076 \pm 0.006$ & $-0.548 \pm 0.041$ & $-0.274 \pm 0.021$ \\
\hline NGC 5145 & $1.636 \pm 0.072$ & $0.619 \pm 0.027$ & $1.552 \pm 0.084$ & $0.587 \pm 0.032$ \\
\hline NGC 5950 & $0.304 \pm 0.037$ & $0.225 \pm 0.027$ & $-0.351 \pm 0.058$ & $-0.26 \pm 0.043$ \\
\hline UGC 10803 & $-0.037 \pm 0.004$ & $-0.029 \pm 0.003$ & $0.036 \pm 0.005$ & $0.028 \pm 0.004$ \\
\hline MCG-01-52-012 & $0.123 \pm 0.017$ & $0.175 \pm 0.025$ & $0.062 \pm 0.029$ & $0.089 \pm 0.041$ \\
\hline UGC 09837 & $-0.027 \pm 0.02$ & $-0.006 \pm 0.004$ & $0.528 \pm 0.038$ & $0.111 \pm 0.008$ \\
\hline UGC 12250 & $-0.491 \pm 0.063$ & $-0.033 \pm 0.004$ & $-0.134 \pm 0.12$ & $-0.009 \pm 0.008$ \\
\hline NGC 5947 & $-0.394 \pm 0.013$ & $-0.057 \pm 0.002$ & $-0.261 \pm 0.036$ & $-0.038 \pm 0.005$ \\
\hline
\end{tabular}


T. Ruiz-Lara et al.: Observational hints of radial migration in disc galaxies from CALIFA

Table B.3. Colour inner gradients $(g-r, g-i$, and $r-i)$ from the analysis of the SDSS images (see Sect. 3.2).

\begin{tabular}{|c|c|c|c|c|c|c|}
\hline \multirow{3}{*}{$\begin{array}{l}\text { Name } \\
\text { (1) }\end{array}$} & \multicolumn{2}{|c|}{$\nabla(g-r)_{\text {in }}$} & \multicolumn{2}{|c|}{$\nabla(g-i)_{\text {in }}$} & \multicolumn{2}{|c|}{$\nabla(r-i)_{\text {in }}$} \\
\hline & $\left(\operatorname{dex} / h_{\mathrm{in}}\right)$ & $(\mathrm{dex} / \mathrm{kpc})$ & $\left(\operatorname{dex} / h_{\text {in }}\right)$ & $(\mathrm{dex} / \mathrm{kpc})$ & $\left(\operatorname{dex} / h_{\text {in }}\right)$ & $(\mathrm{dex} / \mathrm{kpc})$ \\
\hline & (2) & (3) & (4) & (5) & (6) & (7) \\
\hline UGC 00005 & $-0.13 \pm 0.18$ & $-0.02 \pm 0.03$ & $-0.20 \pm 0.18$ & $-0.03 \pm 0.03$ & $-0.07 \pm 0.18$ & $-0.01 \pm 0.03$ \\
\hline NGC 7819 & $-0.33 \pm 0.36$ & $-0.02 \pm 0.02$ & $-0.49 \pm 0.36$ & $-0.03 \pm 0.02$ & $-0.16 \pm 0.36$ & $-0.01 \pm 0.02$ \\
\hline IC 1528 & $-0.10 \pm 0.12$ & $-0.02 \pm 0.03$ & $-0.12 \pm 0.12$ & $-0.03 \pm 0.03$ & $-0.02 \pm 0.12$ & $-0.01 \pm 0.03$ \\
\hline UGC 00036 & $-0.04 \pm 0.16$ & $-0.01 \pm 0.04$ & $-0.05 \pm 0.16$ & $-0.01 \pm 0.04$ & $-0.00 \pm 0.16$ & $-0.00 \pm 0.04$ \\
\hline NGC 0001 & $-0.07 \pm 0.13$ & $-0.02 \pm 0.03$ & $-0.08 \pm 0.13$ & $-0.02 \pm 0.03$ & $-0.02 \pm 0.13$ & $-0.00 \pm 0.03$ \\
\hline NGC 0023 & $-0.33 \pm 0.78$ & $-0.01 \pm 0.02$ & $-0.32 \pm 0.78$ & $-0.01 \pm 0.02$ & $0.02 \pm 0.78$ & $0.00 \pm 0.02$ \\
\hline NGC 0036 & $-0.16 \pm 0.17$ & $-0.02 \pm 0.02$ & $-0.33 \pm 0.17$ & $-0.03 \pm 0.02$ & $-0.17 \pm 0.17$ & $-0.02 \pm 0.02$ \\
\hline UGC 00139 & $-0.19 \pm 0.21$ & $-0.06 \pm 0.06$ & $-0.26 \pm 0.21$ & $-0.08 \pm 0.06$ & $-0.07 \pm 0.21$ & $-0.02 \pm 0.06$ \\
\hline MCG-02-02-030 & $-0.21 \pm 0.59$ & $-0.05 \pm 0.13$ & $-0.31 \pm 0.59$ & $-0.07 \pm 0.13$ & $-0.10 \pm 0.59$ & $-0.02 \pm 0.13$ \\
\hline UGC 00312 & $-0.09 \pm 0.32$ & $-0.02 \pm 0.06$ & $-0.25 \pm 0.32$ & $-0.04 \pm 0.06$ & $-0.16 \pm 0.32$ & $-0.03 \pm 0.06$ \\
\hline ESO540-G003 & $-0.33 \pm 0.87$ & $-0.10 \pm 0.26$ & $-0.54 \pm 0.87$ & $-0.16 \pm 0.26$ & $-0.21 \pm 0.87$ & $-0.06 \pm 0.26$ \\
\hline NGC 0160 & $-0.20 \pm 0.37$ & $-0.01 \pm 0.02$ & $-0.26 \pm 0.37$ & $-0.02 \pm 0.02$ & $-0.06 \pm 0.37$ & $-0.00 \pm 0.02$ \\
\hline NGC 0165 & $-0.16 \pm 0.25$ & $-0.03 \pm 0.04$ & $-0.23 \pm 0.25$ & $-0.04 \pm 0.04$ & $-0.07 \pm 0.25$ & $-0.01 \pm 0.04$ \\
\hline NGC 0171 & $-0.41 \pm 0.56$ & $-0.02 \pm 0.03$ & $-0.49 \pm 0.56$ & $-0.02 \pm 0.03$ & $-0.09 \pm 0.56$ & $-0.00 \pm 0.03$ \\
\hline NGC 0180 & $-0.17 \pm 0.17$ & $-0.02 \pm 0.02$ & $-0.23 \pm 0.17$ & $-0.03 \pm 0.02$ & $-0.07 \pm 0.17$ & $-0.01 \pm 0.02$ \\
\hline NGC 0214 & $-0.11 \pm 0.20$ & $-0.03 \pm 0.05$ & $-0.15 \pm 0.20$ & $-0.04 \pm 0.05$ & $-0.04 \pm 0.20$ & $-0.01 \pm 0.05$ \\
\hline NGC 0237 & $-0.08 \pm 0.11$ & $-0.03 \pm 0.04$ & $-0.09 \pm 0.11$ & $-0.03 \pm 0.04$ & $-0.00 \pm 0.11$ & $-0.00 \pm 0.04$ \\
\hline NGC 0234 & $-0.06 \pm 0.35$ & $-0.01 \pm 0.06$ & $-0.12 \pm 0.35$ & $-0.02 \pm 0.06$ & $-0.05 \pm 0.35$ & $-0.01 \pm 0.06$ \\
\hline NGC 0257 & $-0.10 \pm 0.11$ & $-0.02 \pm 0.02$ & $-0.14 \pm 0.11$ & $-0.02 \pm 0.02$ & $-0.03 \pm 0.11$ & $-0.01 \pm 0.02$ \\
\hline NGC 0309 & $-0.25 \pm 0.20$ & $-0.03 \pm 0.02$ & $-0.31 \pm 0.20$ & $-0.03 \pm 0.02$ & $-0.06 \pm 0.20$ & $-0.01 \pm 0.02$ \\
\hline NGC 0447 & $-0.16 \pm 0.60$ & $-0.02 \pm 0.06$ & $-0.18 \pm 0.60$ & $-0.02 \pm 0.06$ & $-0.02 \pm 0.60$ & $-0.00 \pm 0.06$ \\
\hline NGC 0477 & $-0.23 \pm 0.16$ & $-0.03 \pm 0.02$ & $-0.34 \pm 0.16$ & $-0.05 \pm 0.02$ & $-0.11 \pm 0.16$ & $-0.02 \pm 0.02$ \\
\hline IC 1683 & $-0.07 \pm 0.21$ & $-0.02 \pm 0.04$ & $-0.09 \pm 0.21$ & $-0.02 \pm 0.04$ & $-0.02 \pm 0.21$ & $-0.00 \pm 0.04$ \\
\hline NGC 0496 & $-0.05 \pm 0.13$ & $-0.01 \pm 0.02$ & $-0.06 \pm 0.13$ & $-0.01 \pm 0.02$ & $-0.01 \pm 0.13$ & $-0.00 \pm 0.02$ \\
\hline NGC 0528 & $-0.02 \pm 0.14$ & $-0.01 \pm 0.05$ & $0.01 \pm 0.14$ & $0.00 \pm 0.05$ & $0.02 \pm 0.14$ & $0.01 \pm 0.05$ \\
\hline NGC 0551 & $-0.08 \pm 0.12$ & $-0.01 \pm 0.02$ & $-0.11 \pm 0.12$ & $-0.02 \pm 0.02$ & $-0.03 \pm 0.12$ & $-0.01 \pm 0.02$ \\
\hline NGC 0570 & $-0.04 \pm 0.24$ & $-0.01 \pm 0.04$ & $-0.04 \pm 0.24$ & $-0.01 \pm 0.04$ & $-0.01 \pm 0.24$ & $-0.00 \pm 0.04$ \\
\hline UGC 01271 & $-0.01 \pm 0.19$ & $-0.00 \pm 0.05$ & $0.01 \pm 0.19$ & $0.00 \pm 0.05$ & $0.01 \pm 0.19$ & $0.00 \pm 0.05$ \\
\hline NGC 0716 & $-0.18 \pm 0.19$ & $-0.05 \pm 0.06$ & $-0.30 \pm 0.19$ & $-0.09 \pm 0.06$ & $-0.12 \pm 0.19$ & $-0.04 \pm 0.06$ \\
\hline NGC 0768 & $-0.14 \pm 0.13$ & $-0.02 \pm 0.02$ & $-0.10 \pm 0.13$ & $-0.02 \pm 0.02$ & $0.04 \pm 0.13$ & $0.01 \pm 0.02$ \\
\hline NGC 0776 & $-0.10 \pm 0.19$ & $-0.02 \pm 0.03$ & $-0.14 \pm 0.19$ & $-0.03 \pm 0.03$ & $-0.05 \pm 0.19$ & $-0.01 \pm 0.03$ \\
\hline NGC 0787 & $-0.06 \pm 0.13$ & $-0.01 \pm 0.03$ & $-0.06 \pm 0.13$ & $-0.02 \pm 0.03$ & $-0.01 \pm 0.13$ & $-0.00 \pm 0.03$ \\
\hline NGC 0842 & $-0.01 \pm 0.17$ & $-0.00 \pm 0.04$ & $0.01 \pm 0.17$ & $0.00 \pm 0.04$ & $0.03 \pm 0.17$ & $0.01 \pm 0.04$ \\
\hline UGC 01659 & $-0.63 \pm 2.59$ & $-0.02 \pm 0.09$ & $-0.93 \pm 2.59$ & $-0.03 \pm 0.09$ & $-0.30 \pm 2.59$ & $-0.01 \pm 0.09$ \\
\hline NGC 0873 & $-0.06 \pm 0.16$ & $-0.02 \pm 0.06$ & $-0.04 \pm 0.16$ & $-0.02 \pm 0.06$ & $0.02 \pm 0.16$ & $0.01 \pm 0.06$ \\
\hline NGC 0924 & $-0.08 \pm 0.15$ & $-0.02 \pm 0.03$ & $-0.10 \pm 0.15$ & $-0.02 \pm 0.03$ & $-0.02 \pm 0.15$ & $-0.00 \pm 0.03$ \\
\hline UGC 01918 & $-0.09 \pm 0.87$ & $-0.01 \pm 0.14$ & $-0.34 \pm 0.87$ & $-0.05 \pm 0.14$ & $-0.24 \pm 0.87$ & $-0.04 \pm 0.14$ \\
\hline NGC 0932 & $-0.06 \pm 0.17$ & $-0.01 \pm 0.03$ & $-0.08 \pm 0.17$ & $-0.01 \pm 0.03$ & $-0.02 \pm 0.17$ & $-0.00 \pm 0.03$ \\
\hline NGC 0941 & $-0.16 \pm 0.24$ & $-0.07 \pm 0.11$ & $-0.16 \pm 0.24$ & $-0.07 \pm 0.11$ & $-0.01 \pm 0.24$ & $-0.00 \pm 0.11$ \\
\hline NGC 0976 & $-0.05 \pm 1.68$ & $-0.01 \pm 0.51$ & $-0.07 \pm 1.68$ & $-0.02 \pm 0.51$ & $-0.02 \pm 1.68$ & $-0.01 \pm 0.51$ \\
\hline NGC 0991 & $-0.11 \pm 0.13$ & $-0.04 \pm 0.04$ & $-0.17 \pm 0.13$ & $-0.06 \pm 0.04$ & $-0.06 \pm 0.13$ & $-0.02 \pm 0.04$ \\
\hline UGC 02099 & $-0.10 \pm 0.32$ & $-0.01 \pm 0.03$ & $-0.09 \pm 0.32$ & $-0.01 \pm 0.03$ & $0.01 \pm 0.32$ & $0.00 \pm 0.03$ \\
\hline UGC 02134 & $-0.42 \pm 1.58$ & $-0.02 \pm 0.07$ & $-0.68 \pm 1.58$ & $-0.03 \pm 0.07$ & $-0.26 \pm 1.58$ & $-0.01 \pm 0.07$ \\
\hline NGC 1070 & $-0.04 \pm 0.27$ & $-0.02 \pm 0.11$ & $-0.04 \pm 0.27$ & $-0.02 \pm 0.11$ & $-0.00 \pm 0.27$ & $-0.00 \pm 0.11$ \\
\hline NGC 1094 & $-0.05 \pm 0.24$ & $-0.02 \pm 0.09$ & $-0.06 \pm 0.24$ & $-0.02 \pm 0.09$ & $-0.01 \pm 0.24$ & $-0.00 \pm 0.09$ \\
\hline NGC 1093 & $-0.10 \pm 0.14$ & $-0.02 \pm 0.03$ & $-0.14 \pm 0.14$ & $-0.03 \pm 0.03$ & $-0.04 \pm 0.14$ & $-0.01 \pm 0.03$ \\
\hline UGC 02311 & $-0.08 \pm 0.19$ & $-0.01 \pm 0.03$ & $-0.08 \pm 0.19$ & $-0.01 \pm 0.03$ & $0.00 \pm 0.19$ & $0.00 \pm 0.03$ \\
\hline UGC 02403 & $-0.25 \pm 0.23$ & $-0.05 \pm 0.05$ & $-0.33 \pm 0.23$ & $-0.07 \pm 0.05$ & $-0.08 \pm 0.23$ & $-0.02 \pm 0.05$ \\
\hline UGC 02443 & $-0.13 \pm 0.45$ & $-0.04 \pm 0.12$ & $-0.21 \pm 0.45$ & $-0.06 \pm 0.12$ & $-0.08 \pm 0.45$ & $-0.02 \pm 0.12$ \\
\hline NGC 1167 & $0.00 \pm 0.28$ & $0.00 \pm 0.03$ & $0.04 \pm 0.28$ & $0.00 \pm 0.03$ & $0.04 \pm 0.28$ & $0.00 \pm 0.03$ \\
\hline NGC 1211 & $-0.08 \pm 1.14$ & $-0.01 \pm 0.18$ & $0.04 \pm 1.14$ & $0.01 \pm 0.18$ & $0.11 \pm 1.14$ & $0.02 \pm 0.18$ \\
\hline UGC 02690 & $-0.10 \pm 0.13$ & $-0.02 \pm 0.03$ & $-0.13 \pm 0.13$ & $-0.03 \pm 0.03$ & $-0.03 \pm 0.13$ & $-0.01 \pm 0.03$ \\
\hline MCG-01-10-019 & $-0.14 \pm 0.20$ & $-0.02 \pm 0.03$ & $-0.20 \pm 0.20$ & $-0.03 \pm 0.03$ & $-0.06 \pm 0.20$ & $-0.01 \pm 0.03$ \\
\hline NGC 1645 & $-0.12 \pm 0.15$ & $-0.02 \pm 0.03$ & $-0.14 \pm 0.15$ & $-0.03 \pm 0.03$ & $-0.02 \pm 0.15$ & $-0.00 \pm 0.03$ \\
\hline NGC 1659 & $-0.08 \pm 0.14$ & $-0.02 \pm 0.04$ & $-0.11 \pm 0.14$ & $-0.03 \pm 0.04$ & $-0.02 \pm 0.14$ & $-0.01 \pm 0.04$ \\
\hline NGC 1665 & $-0.00 \pm 0.14$ & $-0.00 \pm 0.04$ & $-0.00 \pm 0.14$ & $-0.00 \pm 0.04$ & $0.00 \pm 0.14$ & $0.00 \pm 0.04$ \\
\hline NGC 1666 & $-0.01 \pm 0.04$ & $0.00 \pm 0.02$ & $0.00 \pm 0.04$ & $0.00 \pm 0.02$ & $0.01 \pm 0.04$ & $0.00 \pm 0.02$ \\
\hline NGC 1667 & $-0.05 \pm 0.11$ & $-0.01 \pm 0.03$ & $-0.05 \pm 0.11$ & $-0.01 \pm 0.03$ & $0.00 \pm 0.11$ & $0.00 \pm 0.03$ \\
\hline UGC 03253 & $-0.17 \pm 0.26$ & $-0.05 \pm 0.07$ & $-0.25 \pm 0.26$ & $-0.07 \pm 0.07$ & $-0.07 \pm 0.26$ & $-0.02 \pm 0.07$ \\
\hline NGC 2253 & $-0.07 \pm 0.11$ & $-0.03 \pm 0.04$ & $-0.11 \pm 0.11$ & $-0.04 \pm 0.04$ & $-0.03 \pm 0.11$ & $-0.01 \pm 0.04$ \\
\hline NGC 2347 & $-0.07 \pm 0.12$ & $-0.01 \pm 0.03$ & $-0.05 \pm 0.12$ & $-0.01 \pm 0.03$ & $0.02 \pm 0.12$ & $0.00 \pm 0.03$ \\
\hline UGC 03944 & $-0.09 \pm 0.13$ & $-0.03 \pm 0.04$ & $-0.14 \pm 0.13$ & $-0.04 \pm 0.04$ & $-0.05 \pm 0.13$ & $-0.02 \pm 0.04$ \\
\hline
\end{tabular}

Notes. This table shows the gradients $\left(\mathrm{dex} / h_{\mathrm{in}}\right.$ and $\left.\mathrm{dex} / \mathrm{kpc}\right)$ for the three colours represented in Fig. 5 . 
Table B.3. continued.

\begin{tabular}{|c|c|c|c|c|c|c|}
\hline \multirow{3}{*}{$\begin{array}{l}\text { Name } \\
\text { (1) }\end{array}$} & \multicolumn{2}{|c|}{$\nabla(g-r)_{\text {in }}$} & \multicolumn{2}{|c|}{$\nabla(g-i)_{\text {in }}$} & \multicolumn{2}{|c|}{$\nabla(r-i)_{\text {in }}$} \\
\hline & $\left(\operatorname{dex} / h_{\text {in }}\right)$ & $(\mathrm{dex} / \mathrm{kpc})$ & $\left(\operatorname{dex} / h_{\text {in }}\right)$ & $(\mathrm{dex} / \mathrm{kpc})$ & $\left(\operatorname{dex} / h_{\mathrm{in}}\right)$ & $(\mathrm{dex} / \mathrm{kpc})$ \\
\hline & & (3) & & & (6) & (7) \\
\hline UGC 03973 & $-0.03 \pm 0.21$ & $-0.00 \pm 0.03$ & $-0.05 \pm 0.21$ & $-0.01 \pm 0.03$ & $-0.02 \pm 0.21$ & $-0.00 \pm 0.03$ \\
\hline UGC 03995 & $-0.13 \pm 0.17$ & $-0.02 \pm 0.02$ & $-0.20 \pm 0.17$ & $-0.03 \pm 0.02$ & $-0.06 \pm 0.17$ & $-0.01 \pm 0.02$ \\
\hline NGC 2449 & $-0.06 \pm 0.12$ & $-0.01 \pm 0.03$ & $-0.06 \pm 0.12$ & $-0.01 \pm 0.03$ & $0.01 \pm 0.12$ & $0.00 \pm 0.03$ \\
\hline NGC 2487 & $-0.27 \pm 0.29$ & $-0.04 \pm 0.04$ & $-0.37 \pm 0.29$ & $-0.05 \pm 0.04$ & $-0.10 \pm 0.29$ & $-0.01 \pm 0.04$ \\
\hline UGC 04195 & $-0.24 \pm 0.24$ & $-0.04 \pm 0.03$ & $-0.34 \pm 0.24$ & $-0.05 \pm 0.03$ & $-0.10 \pm 0.24$ & $-0.01 \pm 0.03$ \\
\hline NGC 2530 & $-0.18 \pm 0.18$ & $-0.04 \pm 0.04$ & $-0.18 \pm 0.18$ & $-0.04 \pm 0.04$ & $0.00 \pm 0.18$ & $0.00 \pm 0.04$ \\
\hline NGC 2540 & $-0.09 \pm 0.17$ & $-0.02 \pm 0.03$ & $-0.13 \pm 0.17$ & $-0.02 \pm 0.03$ & $-0.04 \pm 0.17$ & $-0.01 \pm 0.03$ \\
\hline NGC 2543 & $-0.22 \pm 0.17$ & $-0.06 \pm 0.05$ & $-0.35 \pm 0.17$ & $-0.09 \pm 0.05$ & $-0.13 \pm 0.17$ & $-0.03 \pm 0.05$ \\
\hline UGC 04308 & $-0.18 \pm 0.20$ & $-0.04 \pm 0.05$ & $-0.25 \pm 0.20$ & $-0.06 \pm 0.05$ & $-0.07 \pm 0.20$ & $-0.02 \pm 0.05$ \\
\hline NGC 2553 & $-0.02 \pm 0.19$ & $-0.01 \pm 0.04$ & $-0.00 \pm 0.19$ & $-0.00 \pm 0.04$ & $0.02 \pm 0.19$ & $0.00 \pm 0.04$ \\
\hline UGC 04262 & $-0.13 \pm 0.17$ & $-0.02 \pm 0.03$ & $-0.20 \pm 0.17$ & $-0.03 \pm 0.03$ & $-0.07 \pm 0.17$ & $-0.01 \pm 0.03$ \\
\hline NGC 2558 & $-0.13 \pm 0.21$ & $-0.02 \pm 0.04$ & $-0.18 \pm 0.21$ & $-0.03 \pm 0.04$ & $-0.04 \pm 0.21$ & $-0.01 \pm 0.04$ \\
\hline NGC 2565 & $-0.12 \pm 0.14$ & $-0.03 \pm 0.04$ & $-0.17 \pm 0.14$ & $-0.05 \pm 0.04$ & $-0.04 \pm 0.14$ & $-0.01 \pm 0.04$ \\
\hline NGC 2572 & $-0.07 \pm 0.18$ & $-0.02 \pm 0.04$ & $-0.08 \pm 0.18$ & $-0.02 \pm 0.04$ & $-0.01 \pm 0.18$ & $-0.00 \pm 0.04$ \\
\hline UGC 04375 & $-0.12 \pm 0.15$ & $-0.04 \pm 0.05$ & $-0.17 \pm 0.15$ & $-0.06 \pm 0.05$ & $-0.05 \pm 0.15$ & $-0.02 \pm 0.05$ \\
\hline NGC 2596 & $-0.34 \pm 0.33$ & $-0.04 \pm 0.04$ & $-0.50 \pm 0.33$ & $-0.06 \pm 0.04$ & $-0.16 \pm 0.33$ & $-0.02 \pm 0.04$ \\
\hline NGC 2595 & $-0.13 \pm 0.29$ & $-0.02 \pm 0.03$ & $-0.26 \pm 0.29$ & $-0.03 \pm 0.03$ & $-0.13 \pm 0.29$ & $-0.01 \pm 0.03$ \\
\hline NGC 2604 & $-0.08 \pm 0.25$ & $-0.03 \pm 0.09$ & $-0.09 \pm 0.25$ & $-0.03 \pm 0.09$ & $-0.02 \pm 0.25$ & $-0.01 \pm 0.09$ \\
\hline NGC 2639 & $-0.04 \pm 0.09$ & $-0.01 \pm 0.03$ & $-0.04 \pm 0.09$ & $-0.01 \pm 0.03$ & $-0.00 \pm 0.09$ & $-0.00 \pm 0.03$ \\
\hline NGC 2730 & $-0.16 \pm 0.32$ & $-0.03 \pm 0.05$ & $-0.29 \pm 0.32$ & $-0.05 \pm 0.05$ & $-0.13 \pm 0.32$ & $-0.02 \pm 0.05$ \\
\hline NGC 2805 & $-0.11 \pm 4.02$ & $-0.02 \pm 0.59$ & $-0.12 \pm 4.02$ & $-0.02 \pm 0.59$ & $-0.01 \pm 4.02$ & $-0.00 \pm 0.59$ \\
\hline NGC 2906 & $-0.05 \pm 0.10$ & $-0.03 \pm 0.05$ & $-0.06 \pm 0.10$ & $-0.03 \pm 0.05$ & $-0.00 \pm 0.10$ & $-0.00 \pm 0.05$ \\
\hline NGC 2916 & $-0.23 \pm 0.30$ & $-0.04 \pm 0.05$ & $-0.33 \pm 0.30$ & $-0.06 \pm 0.05$ & $-0.10 \pm 0.30$ & $-0.02 \pm 0.05$ \\
\hline UGC 05108 & $-0.56 \pm 0.85$ & $-0.01 \pm 0.02$ & $-0.61 \pm 0.85$ & $-0.02 \pm 0.02$ & $-0.05 \pm 0.85$ & $-0.00 \pm 0.02$ \\
\hline UGC 05359 & $-0.15 \pm 0.17$ & $-0.02 \pm 0.03$ & $-0.21 \pm 0.17$ & $-0.03 \pm 0.03$ & $-0.06 \pm 0.17$ & $-0.01 \pm 0.03$ \\
\hline UGC 05396 & $-0.14 \pm 0.08$ & $-0.02 \pm 0.01$ & $-0.14 \pm 0.08$ & $-0.02 \pm 0.01$ & $0.00 \pm 0.08$ & $0.00 \pm 0.01$ \\
\hline NGC 3106 & $-0.07 \pm 0.21$ & $-0.01 \pm 0.03$ & $-0.08 \pm 0.21$ & $-0.01 \pm 0.03$ & $-0.01 \pm 0.21$ & $-0.00 \pm 0.03$ \\
\hline NGC 3300 & $-0.01 \pm 0.15$ & $-0.00 \pm 0.05$ & $-0.01 \pm 0.15$ & $-0.00 \pm 0.05$ & $0.01 \pm 0.15$ & $0.00 \pm 0.05$ \\
\hline NGC 3381 & $-0.06 \pm 0.16$ & $-0.03 \pm 0.08$ & $-0.05 \pm 0.16$ & $-0.02 \pm 0.08$ & $0.01 \pm 0.16$ & $0.00 \pm 0.08$ \\
\hline IC 0674 & $-0.12 \pm 0.11$ & $-0.02 \pm 0.02$ & $-0.16 \pm 0.11$ & $-0.03 \pm 0.02$ & $-0.04 \pm 0.11$ & $-0.01 \pm 0.02$ \\
\hline UGC 06312 & $-0.10 \pm 0.23$ & $-0.01 \pm 0.03$ & $-0.15 \pm 0.23$ & $-0.02 \pm 0.03$ & $-0.04 \pm 0.23$ & $-0.01 \pm 0.03$ \\
\hline NGC 3614 & $-0.21 \pm 0.32$ & $-0.04 \pm 0.07$ & $-0.31 \pm 0.32$ & $-0.06 \pm 0.07$ & $-0.10 \pm 0.32$ & $-0.02 \pm 0.07$ \\
\hline NGC 3687 & $-0.11 \pm 0.15$ & $-0.05 \pm 0.07$ & $-0.15 \pm 0.15$ & $-0.07 \pm 0.07$ & $-0.04 \pm 0.15$ & $-0.02 \pm 0.07$ \\
\hline NGC 3811 & $-0.09 \pm 0.12$ & $-0.04 \pm 0.05$ & $-0.13 \pm 0.12$ & $-0.05 \pm 0.05$ & $-0.03 \pm 0.12$ & $-0.01 \pm 0.05$ \\
\hline NGC 3815 & $-0.10 \pm 0.11$ & $-0.04 \pm 0.04$ & $-0.13 \pm 0.11$ & $-0.04 \pm 0.04$ & $-0.02 \pm 0.11$ & $-0.01 \pm 0.04$ \\
\hline NGC 3994 & $-0.01 \pm 0.14$ & $-0.01 \pm 0.11$ & $0.03 \pm 0.14$ & $0.02 \pm 0.11$ & $0.04 \pm 0.14$ & $0.03 \pm 0.11$ \\
\hline NGC 4003 & $-0.02 \pm 0.19$ & $-0.00 \pm 0.03$ & $-0.02 \pm 0.19$ & $-0.00 \pm 0.03$ & $-0.00 \pm 0.19$ & $-0.00 \pm 0.03$ \\
\hline UGC 07012 & $-0.06 \pm 0.23$ & $-0.04 \pm 0.13$ & $-0.09 \pm 0.23$ & $-0.05 \pm 0.13$ & $-0.03 \pm 0.23$ & $-0.02 \pm 0.13$ \\
\hline NGC 4047 & $-0.05 \pm 0.11$ & $-0.02 \pm 0.04$ & $-0.05 \pm 0.11$ & $-0.02 \pm 0.04$ & $-0.00 \pm 0.11$ & $-0.00 \pm 0.04$ \\
\hline UGC 07145 & $-0.16 \pm 0.38$ & $-0.03 \pm 0.06$ & $-0.23 \pm 0.38$ & $-0.04 \pm 0.06$ & $-0.06 \pm 0.38$ & $-0.01 \pm 0.06$ \\
\hline NGC 4185 & $-0.13 \pm 0.30$ & $-0.02 \pm 0.04$ & $-0.20 \pm 0.30$ & $-0.03 \pm 0.04$ & $-0.07 \pm 0.30$ & $-0.01 \pm 0.04$ \\
\hline NGC 4210 & $-0.22 \pm 0.32$ & $-0.04 \pm 0.06$ & $-0.33 \pm 0.32$ & $-0.06 \pm 0.06$ & $-0.12 \pm 0.32$ & $-0.02 \pm 0.06$ \\
\hline NGC 4470 & $0.07 \pm 0.13$ & $0.04 \pm 0.07$ & $0.12 \pm 0.13$ & $0.07 \pm 0.07$ & $0.04 \pm 0.13$ & $0.02 \pm 0.07$ \\
\hline NGC 4711 & $-0.14 \pm 0.32$ & $-0.03 \pm 0.08$ & $-0.20 \pm 0.32$ & $-0.05 \pm 0.08$ & $-0.06 \pm 0.32$ & $-0.02 \pm 0.08$ \\
\hline UGC 08004 & $-0.14 \pm 0.13$ & $-0.02 \pm 0.02$ & $-0.21 \pm 0.13$ & $-0.03 \pm 0.02$ & $-0.08 \pm 0.13$ & $-0.01 \pm 0.02$ \\
\hline NGC 4961 & $-0.10 \pm 0.32$ & $-0.04 \pm 0.13$ & $-0.11 \pm 0.32$ & $-0.04 \pm 0.13$ & $-0.00 \pm 0.32$ & $-0.00 \pm 0.13$ \\
\hline UGC 08231 & $-0.04 \pm 0.12$ & $-0.02 \pm 0.05$ & $-0.06 \pm 0.12$ & $-0.03 \pm 0.05$ & $-0.02 \pm 0.12$ & $-0.01 \pm 0.05$ \\
\hline NGC 5000 & $-0.10 \pm 0.20$ & $-0.02 \pm 0.04$ & $-0.11 \pm 0.20$ & $-0.02 \pm 0.04$ & $-0.01 \pm 0.20$ & $-0.00 \pm 0.04$ \\
\hline NGC 5016 & $-0.05 \pm 0.14$ & $-0.03 \pm 0.07$ & $-0.06 \pm 0.14$ & $-0.03 \pm 0.07$ & $-0.01 \pm 0.14$ & $-0.01 \pm 0.07$ \\
\hline NGC 5157 & $-0.09 \pm 0.16$ & $-0.01 \pm 0.02$ & $-0.12 \pm 0.16$ & $-0.02 \pm 0.02$ & $-0.02 \pm 0.16$ & $-0.00 \pm 0.02$ \\
\hline NGC 5205 & $-0.14 \pm 0.17$ & $-0.08 \pm 0.09$ & $-0.17 \pm 0.17$ & $-0.10 \pm 0.09$ & $-0.03 \pm 0.17$ & $-0.02 \pm 0.09$ \\
\hline NGC 5267 & $-0.26 \pm 0.55$ & $-0.03 \pm 0.06$ & $-0.32 \pm 0.55$ & $-0.03 \pm 0.06$ & $-0.06 \pm 0.55$ & $-0.01 \pm 0.06$ \\
\hline NGC 5320 & $-0.18 \pm 0.16$ & $-0.05 \pm 0.04$ & $-0.28 \pm 0.16$ & $-0.07 \pm 0.04$ & $-0.09 \pm 0.16$ & $-0.02 \pm 0.04$ \\
\hline UGC 08781 & $-0.15 \pm 0.17$ & $-0.02 \pm 0.02$ & $-0.20 \pm 0.17$ & $-0.03 \pm 0.02$ & $-0.05 \pm 0.17$ & $-0.01 \pm 0.02$ \\
\hline NGC 5376 & $-0.17 \pm 0.35$ & $-0.06 \pm 0.12$ & $-0.24 \pm 0.35$ & $-0.08 \pm 0.12$ & $-0.06 \pm 0.35$ & $-0.02 \pm 0.12$ \\
\hline NGC 5379 & $-0.03 \pm 0.17$ & $-0.02 \pm 0.09$ & $-0.02 \pm 0.17$ & $-0.01 \pm 0.09$ & $0.01 \pm 0.17$ & $0.01 \pm 0.09$ \\
\hline NGC 5378 & $-0.14 \pm 0.23$ & $-0.03 \pm 0.04$ & $-0.19 \pm 0.23$ & $-0.04 \pm 0.04$ & $-0.05 \pm 0.23$ & $-0.01 \pm 0.04$ \\
\hline NGC 5406 & $-0.35 \pm 1.00$ & $-0.02 \pm 0.05$ & $-0.63 \pm 1.00$ & $-0.03 \pm 0.05$ & $-0.28 \pm 1.00$ & $-0.01 \pm 0.05$ \\
\hline NGC 5473 & $-0.02 \pm 0.29$ & $-0.01 \pm 0.12$ & $-0.01 \pm 0.29$ & $-0.00 \pm 0.12$ & $0.02 \pm 0.29$ & $0.01 \pm 0.12$ \\
\hline NGC 5480 & $-0.14 \pm 0.52$ & $-0.05 \pm 0.18$ & $-0.22 \pm 0.52$ & $-0.07 \pm 0.18$ & $-0.08 \pm 0.52$ & $-0.03 \pm 0.18$ \\
\hline UGC 09067 & $-0.11 \pm 0.16$ & $-0.02 \pm 0.03$ & $-0.13 \pm 0.16$ & $-0.03 \pm 0.03$ & $-0.02 \pm 0.16$ & $-0.00 \pm 0.03$ \\
\hline NGC 5520 & $-0.10 \pm 0.10$ & $-0.06 \pm 0.06$ & $-0.16 \pm 0.10$ & $-0.09 \pm 0.06$ & $-0.06 \pm 0.10$ & $-0.04 \pm 0.06$ \\
\hline NGC 5633 & $-0.02 \pm 0.13$ & $-0.02 \pm 0.09$ & $-0.03 \pm 0.13$ & $-0.02 \pm 0.09$ & $-0.01 \pm 0.13$ & $-0.00 \pm 0.09$ \\
\hline
\end{tabular}


T. Ruiz-Lara et al.: Observational hints of radial migration in disc galaxies from CALIFA

Table B.3. continued.

\begin{tabular}{|c|c|c|c|c|c|c|}
\hline \multirow{3}{*}{$\begin{array}{l}\text { Name } \\
\text { (1) }\end{array}$} & \multicolumn{2}{|c|}{$\nabla(g-r)_{\text {in }}$} & \multicolumn{2}{|c|}{$\nabla(g-i)_{\text {in }}$} & \multicolumn{2}{|c|}{$\nabla(r-i)_{\text {in }}$} \\
\hline & $\left(\operatorname{dex} / h_{\text {in }}\right)$ & $(\mathrm{dex} / \mathrm{kpc})$ & $\left(\operatorname{dex} / h_{\text {in }}\right)$ & (dex/kpc) & $\left(\operatorname{dex} / h_{\text {in }}\right)$ & $(\mathrm{dex} / \mathrm{kpc})$ \\
\hline & (2) & (3) & (4) & (5) & (6) & (7) \\
\hline NGC 5657 & $-0.13 \pm 0.11$ & $-0.03 \pm 0.03$ & $-0.17 \pm 0.11$ & $-0.04 \pm 0.03$ & $-0.05 \pm 0.11$ & $-0.01 \pm 0.03$ \\
\hline NGC 5720 & $-0.14 \pm 0.12$ & $-0.02 \pm 0.02$ & $-0.17 \pm 0.12$ & $-0.03 \pm 0.02$ & $-0.03 \pm 0.12$ & $-0.00 \pm 0.02$ \\
\hline NGC 5732 & $-0.08 \pm 0.12$ & $-0.03 \pm 0.05$ & $-0.07 \pm 0.12$ & $-0.03 \pm 0.05$ & $0.01 \pm 0.12$ & $0.01 \pm 0.05$ \\
\hline UGC 09476 & $-0.07 \pm 0.16$ & $-0.02 \pm 0.04$ & $-0.06 \pm 0.16$ & $-0.01 \pm 0.04$ & $0.01 \pm 0.16$ & $0.00 \pm 0.04$ \\
\hline NGC 5784 & $-0.02 \pm 0.20$ & $-0.00 \pm 0.03$ & $-0.03 \pm 0.20$ & $-0.00 \pm 0.03$ & $-0.01 \pm 0.20$ & $-0.00 \pm 0.03$ \\
\hline UGC 09598 & $-0.16 \pm 0.21$ & $-0.03 \pm 0.04$ & $-0.22 \pm 0.21$ & $-0.04 \pm 0.04$ & $-0.06 \pm 0.21$ & $-0.01 \pm 0.04$ \\
\hline NGC 5876 & $-0.11 \pm 0.21$ & $-0.01 \pm 0.03$ & $-0.14 \pm 0.21$ & $-0.02 \pm 0.03$ & $-0.03 \pm 0.21$ & $-0.00 \pm 0.03$ \\
\hline NGC 5888 & $0.06 \pm 0.11$ & $0.01 \pm 0.01$ & $0.07 \pm 0.11$ & $0.01 \pm 0.01$ & $0.02 \pm 0.11$ & $0.00 \pm 0.01$ \\
\hline UGC 09777 & $-0.10 \pm 0.10$ & $-0.04 \pm 0.04$ & $-0.16 \pm 0.10$ & $-0.06 \pm 0.04$ & $-0.05 \pm 0.10$ & $-0.02 \pm 0.04$ \\
\hline UGC 09842 & $-0.09 \pm 0.17$ & $-0.01 \pm 0.03$ & $-0.26 \pm 0.17$ & $-0.04 \pm 0.03$ & $-0.17 \pm 0.17$ & $-0.03 \pm 0.03$ \\
\hline NGC 5957 & $-0.26 \pm 0.18$ & $-0.10 \pm 0.07$ & $-0.32 \pm 0.18$ & $-0.13 \pm 0.07$ & $-0.06 \pm 0.18$ & $-0.02 \pm 0.07$ \\
\hline NGC 5971 & $-0.21 \pm 0.23$ & $-0.05 \pm 0.05$ & $-0.22 \pm 0.23$ & $-0.05 \pm 0.05$ & $-0.01 \pm 0.23$ & $-0.00 \pm 0.05$ \\
\hline IC 4566 & $-0.10 \pm 0.18$ & $-0.02 \pm 0.04$ & $-0.13 \pm 0.18$ & $-0.03 \pm 0.04$ & $-0.03 \pm 0.18$ & $-0.01 \pm 0.04$ \\
\hline NGC 5980 & $-0.13 \pm 0.11$ & $-0.04 \pm 0.03$ & $-0.18 \pm 0.11$ & $-0.05 \pm 0.03$ & $-0.04 \pm 0.11$ & $-0.01 \pm 0.03$ \\
\hline NGC 6004 & $-0.05 \pm 0.14$ & $-0.01 \pm 0.03$ & $-0.05 \pm 0.14$ & $-0.01 \pm 0.03$ & $-0.00 \pm 0.14$ & $-0.00 \pm 0.03$ \\
\hline IC 1151 & $-0.13 \pm 0.13$ & $-0.05 \pm 0.05$ & $-0.16 \pm 0.13$ & $-0.06 \pm 0.05$ & $-0.03 \pm 0.13$ & $-0.01 \pm 0.05$ \\
\hline NGC 6032 & $-0.28 \pm 1.00$ & $-0.01 \pm 0.03$ & $-0.74 \pm 1.00$ & $-0.02 \pm 0.03$ & $-0.46 \pm 1.00$ & $-0.02 \pm 0.03$ \\
\hline NGC 6060 & $-0.17 \pm 0.16$ & $-0.03 \pm 0.02$ & $-0.26 \pm 0.16$ & $-0.04 \pm 0.02$ & $-0.09 \pm 0.16$ & $-0.01 \pm 0.02$ \\
\hline NGC 6063 & $-0.17 \pm 0.36$ & $-0.04 \pm 0.08$ & $-0.27 \pm 0.36$ & $-0.06 \pm 0.08$ & $-0.10 \pm 0.36$ & $-0.02 \pm 0.08$ \\
\hline IC 1199 & $-0.33 \pm 0.41$ & $-0.06 \pm 0.07$ & $-0.38 \pm 0.41$ & $-0.07 \pm 0.07$ & $-0.05 \pm 0.41$ & $-0.01 \pm 0.07$ \\
\hline NGC 6154 & $-0.14 \pm 0.20$ & $-0.02 \pm 0.03$ & $-0.20 \pm 0.20$ & $-0.03 \pm 0.03$ & $-0.05 \pm 0.20$ & $-0.01 \pm 0.03$ \\
\hline NGC 6155 & $-0.02 \pm 0.13$ & $-0.01 \pm 0.07$ & $-0.02 \pm 0.13$ & $-0.01 \pm 0.07$ & $-0.00 \pm 0.13$ & $-0.00 \pm 0.07$ \\
\hline NGC 6186 & $-0.16 \pm 0.45$ & $-0.02 \pm 0.06$ & $-0.13 \pm 0.45$ & $-0.02 \pm 0.06$ & $0.03 \pm 0.45$ & $0.00 \pm 0.06$ \\
\hline NGC 6278 & $-0.01 \pm 0.28$ & $-0.00 \pm 0.06$ & $0.02 \pm 0.28$ & $0.00 \pm 0.06$ & $0.03 \pm 0.28$ & $0.01 \pm 0.06$ \\
\hline NGC 6301 & $-0.20 \pm 0.28$ & $-0.02 \pm 0.02$ & $-0.32 \pm 0.28$ & $-0.03 \pm 0.02$ & $-0.12 \pm 0.28$ & $-0.01 \pm 0.02$ \\
\hline NGC 6314 & $-0.09 \pm 0.20$ & $-0.01 \pm 0.03$ & $-0.12 \pm 0.20$ & $-0.02 \pm 0.03$ & $-0.03 \pm 0.20$ & $-0.00 \pm 0.03$ \\
\hline UGC 10796 & $0.18 \pm 0.31$ & $0.02 \pm 0.04$ & $0.24 \pm 0.31$ & $0.03 \pm 0.04$ & $0.06 \pm 0.31$ & $0.01 \pm 0.04$ \\
\hline UGC 10811 & $-0.18 \pm 0.19$ & $-0.03 \pm 0.03$ & $-0.23 \pm 0.19$ & $-0.03 \pm 0.03$ & $-0.05 \pm 0.19$ & $-0.01 \pm 0.03$ \\
\hline IC 1256 & $-0.08 \pm 0.19$ & $-0.02 \pm 0.05$ & $-0.10 \pm 0.19$ & $-0.03 \pm 0.05$ & $-0.03 \pm 0.19$ & $-0.01 \pm 0.05$ \\
\hline NGC 6394 & $-0.09 \pm 0.16$ & $-0.01 \pm 0.02$ & $-0.13 \pm 0.16$ & $-0.02 \pm 0.02$ & $-0.04 \pm 0.16$ & $-0.01 \pm 0.02$ \\
\hline UGC 10905 & $-0.07 \pm 0.24$ & $-0.01 \pm 0.02$ & $-0.07 \pm 0.24$ & $-0.01 \pm 0.02$ & $-0.00 \pm 0.24$ & $-0.00 \pm 0.02$ \\
\hline NGC 6427 & $-0.08 \pm 0.12$ & $-0.03 \pm 0.04$ & $-0.04 \pm 0.12$ & $-0.01 \pm 0.04$ & $0.04 \pm 0.12$ & $0.01 \pm 0.04$ \\
\hline NGC 6478 & $-0.17 \pm 0.43$ & $-0.02 \pm 0.05$ & $-0.30 \pm 0.43$ & $-0.03 \pm 0.05$ & $-0.12 \pm 0.43$ & $-0.01 \pm 0.05$ \\
\hline NGC 6497 & $-0.20 \pm 0.42$ & $-0.02 \pm 0.05$ & $-0.30 \pm 0.42$ & $-0.04 \pm 0.05$ & $-0.10 \pm 0.42$ & $-0.01 \pm 0.05$ \\
\hline UGC 11228 & $-0.03 \pm 0.17$ & $-0.01 \pm 0.03$ & $-0.01 \pm 0.17$ & $-0.00 \pm 0.03$ & $0.02 \pm 0.17$ & $0.00 \pm 0.03$ \\
\hline UGC 11262 & $-0.20 \pm 0.27$ & $-0.03 \pm 0.04$ & $-0.27 \pm 0.27$ & $-0.04 \pm 0.04$ & $-0.07 \pm 0.27$ & $-0.01 \pm 0.04$ \\
\hline MCG-02-51-004 & $-0.16 \pm 0.17$ & $-0.03 \pm 0.03$ & $-0.25 \pm 0.17$ & $-0.05 \pm 0.03$ & $-0.09 \pm 0.17$ & $-0.02 \pm 0.03$ \\
\hline NGC 6941 & $-0.16 \pm 0.17$ & $-0.02 \pm 0.02$ & $-0.21 \pm 0.17$ & $-0.03 \pm 0.02$ & $-0.05 \pm 0.17$ & $-0.01 \pm 0.02$ \\
\hline NGC 6945 & $-0.00 \pm 0.22$ & $-0.00 \pm 0.05$ & $0.01 \pm 0.22$ & $0.00 \pm 0.05$ & $0.02 \pm 0.22$ & $0.00 \pm 0.05$ \\
\hline NGC 6978 & $-0.04 \pm 0.12$ & $-0.01 \pm 0.02$ & $-0.03 \pm 0.12$ & $-0.01 \pm 0.02$ & $0.00 \pm 0.12$ & $0.00 \pm 0.02$ \\
\hline UGC 11649 & $-0.12 \pm 0.19$ & $-0.03 \pm 0.05$ & $-0.15 \pm 0.19$ & $-0.04 \pm 0.05$ & $-0.03 \pm 0.19$ & $-0.01 \pm 0.05$ \\
\hline NGC 7047 & $-0.19 \pm 0.41$ & $-0.03 \pm 0.06$ & $-0.25 \pm 0.41$ & $-0.04 \pm 0.06$ & $-0.06 \pm 0.41$ & $-0.01 \pm 0.06$ \\
\hline NGC 7311 & $-0.08 \pm 0.12$ & $-0.02 \pm 0.03$ & $-0.10 \pm 0.12$ & $-0.03 \pm 0.03$ & $-0.02 \pm 0.12$ & $-0.01 \pm 0.03$ \\
\hline NGC 7321 & $-0.11 \pm 0.12$ & $-0.02 \pm 0.02$ & $-0.14 \pm 0.12$ & $-0.03 \pm 0.02$ & $-0.03 \pm 0.12$ & $-0.01 \pm 0.02$ \\
\hline UGC 12185 & $-0.22 \pm 0.43$ & $-0.02 \pm 0.04$ & $-0.25 \pm 0.43$ & $-0.02 \pm 0.04$ & $-0.03 \pm 0.43$ & $-0.00 \pm 0.04$ \\
\hline UGC 12224 & $-0.22 \pm 0.28$ & $-0.05 \pm 0.06$ & $-0.28 \pm 0.28$ & $-0.06 \pm 0.06$ & $-0.06 \pm 0.28$ & $-0.01 \pm 0.06$ \\
\hline NGC 7466 & $-0.11 \pm 0.15$ & $-0.02 \pm 0.02$ & $-0.20 \pm 0.15$ & $-0.03 \pm 0.02$ & $-0.09 \pm 0.15$ & $-0.01 \pm 0.02$ \\
\hline NGC 7489 & $-0.13 \pm 0.13$ & $-0.02 \pm 0.02$ & $-0.20 \pm 0.13$ & $-0.04 \pm 0.02$ & $-0.06 \pm 0.13$ & $-0.01 \pm 0.02$ \\
\hline NGC 7536 & $-0.20 \pm 0.63$ & $-0.02 \pm 0.07$ & $-0.38 \pm 0.63$ & $-0.04 \pm 0.07$ & $-0.18 \pm 0.63$ & $-0.02 \pm 0.07$ \\
\hline NGC 7549 & $-0.22 \pm 0.26$ & $-0.03 \pm 0.03$ & $-0.41 \pm 0.26$ & $-0.05 \pm 0.03$ & $-0.19 \pm 0.26$ & $-0.02 \pm 0.03$ \\
\hline NGC 7563 & $-0.05 \pm 1.45$ & $-0.00 \pm 0.08$ & $0.08 \pm 1.45$ & $0.00 \pm 0.08$ & $0.13 \pm 1.45$ & $0.01 \pm 0.08$ \\
\hline NGC 7591 & $-0.15 \pm 0.14$ & $-0.02 \pm 0.02$ & $-0.18 \pm 0.14$ & $-0.03 \pm 0.02$ & $-0.04 \pm 0.14$ & $-0.01 \pm 0.02$ \\
\hline IC 5309 & $-0.08 \pm 0.18$ & $-0.02 \pm 0.05$ & $-0.09 \pm 0.18$ & $-0.03 \pm 0.05$ & $-0.01 \pm 0.18$ & $-0.00 \pm 0.05$ \\
\hline NGC 7611 & $-0.02 \pm 0.22$ & $-0.01 \pm 0.08$ & $-0.01 \pm 0.22$ & $-0.00 \pm 0.08$ & $0.01 \pm 0.22$ & $0.00 \pm 0.08$ \\
\hline NGC 7623 & $0.00 \pm 0.48$ & $0.00 \pm 0.09$ & $0.04 \pm 0.48$ & $0.01 \pm 0.09$ & $0.04 \pm 0.48$ & $0.01 \pm 0.09$ \\
\hline NGC 7631 & $-0.12 \pm 0.14$ & $-0.03 \pm 0.04$ & $-0.16 \pm 0.14$ & $-0.04 \pm 0.04$ & $-0.04 \pm 0.14$ & $-0.01 \pm 0.04$ \\
\hline NGC 7653 & $-0.08 \pm 0.16$ & $-0.02 \pm 0.04$ & $-0.12 \pm 0.16$ & $-0.03 \pm 0.04$ & $-0.04 \pm 0.16$ & $-0.01 \pm 0.04$ \\
\hline NGC 7671 & $-0.02 \pm 0.08$ & $-0.01 \pm 0.03$ & $-0.00 \pm 0.08$ & $-0.00 \pm 0.03$ & $0.02 \pm 0.08$ & $0.01 \pm 0.03$ \\
\hline NGC 7691 & $-0.15 \pm 0.26$ & $-0.03 \pm 0.05$ & $-0.20 \pm 0.26$ & $-0.04 \pm 0.05$ & $-0.06 \pm 0.26$ & $-0.01 \pm 0.05$ \\
\hline NGC 7716 & $-0.15 \pm 0.14$ & $-0.03 \pm 0.03$ & $-0.20 \pm 0.14$ & $-0.04 \pm 0.03$ & $-0.05 \pm 0.14$ & $-0.01 \pm 0.03$ \\
\hline NGC 7722 & $-0.09 \pm 0.27$ & $-0.03 \pm 0.09$ & $-0.16 \pm 0.27$ & $-0.05 \pm 0.09$ & $-0.06 \pm 0.27$ & $-0.02 \pm 0.09$ \\
\hline NGC 7738 & $-0.07 \pm 0.13$ & $-0.02 \pm 0.03$ & $-0.11 \pm 0.13$ & $-0.02 \pm 0.03$ & $-0.03 \pm 0.13$ & $-0.01 \pm 0.03$ \\
\hline UGC 12810 & $-0.30 \pm 0.17$ & $-0.04 \pm 0.02$ & $-0.37 \pm 0.17$ & $-0.04 \pm 0.02$ & $-0.07 \pm 0.17$ & $-0.01 \pm 0.02$ \\
\hline
\end{tabular}


Table B.3. continued.

\begin{tabular}{|c|c|c|c|c|c|c|}
\hline $\begin{array}{l}\text { Name } \\
\text { (1) } \\
\end{array}$ & $\begin{array}{r}\nabla( \\
\left(\operatorname{dex} / h_{\text {in }}\right) \\
(2) \\
\end{array}$ & $\begin{array}{r}\text { in } \\
(\mathrm{dex} / \mathrm{kpc}) \\
(3)\end{array}$ & $\begin{array}{r}\nabla\left(\operatorname{dex} / h_{\text {in }}\right) \\
(4)\end{array}$ & $\begin{array}{r}\text { in } \\
\text { (dex/kpc) } \\
(5) \\
\end{array}$ & $\begin{array}{r}\nabla \\
\left(\operatorname{dex} / h_{\text {in }}\right) \\
(6) \\
\end{array}$ & $\begin{array}{r}\text { in } \\
(\mathrm{dex} / \mathrm{kpc}) \\
(7) \\
\end{array}$ \\
\hline UGC 12816 & $-0.15 \pm 0.29$ & $-0.01 \pm 0.02$ & $-0.21 \pm 0.29$ & $-0.02 \pm 0.02$ & $-0.06 \pm 0.29$ & $-0.01 \pm 0.02$ \\
\hline NGC 7782 & $-0.16 \pm 0.17$ & $-0.02 \pm 0.03$ & $-0.22 \pm 0.17$ & $-0.03 \pm 0.03$ & $-0.06 \pm 0.17$ & $-0.01 \pm 0.03$ \\
\hline NGC 7787 & $-0.02 \pm 0.20$ & $-0.00 \pm 0.04$ & $-0.22 \pm 0.20$ & $-0.05 \pm 0.04$ & $-0.20 \pm 0.20$ & $-0.04 \pm 0.04$ \\
\hline UGC 12864 & $-0.16 \pm 0.20$ & $-0.01 \pm 0.02$ & $-0.23 \pm 0.20$ & $-0.02 \pm 0.02$ & $-0.06 \pm 0.20$ & $-0.01 \pm 0.02$ \\
\hline UGC 04455 & $0.22 \pm 0.49$ & $0.06 \pm 0.14$ & $-0.06 \pm 0.49$ & $-0.02 \pm 0.14$ & $-0.27 \pm 0.49$ & $-0.08 \pm 0.14$ \\
\hline UGC 06249 & $0.13 \pm 0.57$ & $0.01 \pm 0.05$ & $-0.04 \pm 0.57$ & $-0.00 \pm 0.05$ & $-0.17 \pm 0.57$ & $-0.01 \pm 0.05$ \\
\hline SN2002ji & $-0.13 \pm 0.28$ & $-0.14 \pm 0.29$ & $-0.22 \pm 0.28$ & $-0.23 \pm 0.29$ & $-0.09 \pm 0.28$ & $-0.09 \pm 0.29$ \\
\hline UGC 07129 & $-0.03 \pm 0.34$ & $-0.03 \pm 0.38$ & $0.04 \pm 0.34$ & $0.05 \pm 0.38$ & $0.07 \pm 0.34$ & $0.08 \pm 0.38$ \\
\hline UGC 08909 & $0.10 \pm 0.45$ & $0.12 \pm 0.54$ & $-0.05 \pm 0.45$ & $-0.06 \pm 0.54$ & $-0.14 \pm 0.45$ & $-0.17 \pm 0.54$ \\
\hline NGC 0495 & $0.02 \pm 0.17$ & $0.01 \pm 0.13$ & $0.02 \pm 0.17$ & $0.01 \pm 0.13$ & $-0.00 \pm 0.17$ & $-0.00 \pm 0.13$ \\
\hline KUG1349+143 & $0.08 \pm 0.40$ & $0.01 \pm 0.05$ & $-0.03 \pm 0.40$ & $-0.00 \pm 0.05$ & $-0.11 \pm 0.40$ & $-0.02 \pm 0.05$ \\
\hline CGCG163-062 & $0.04 \pm 0.64$ & $0.01 \pm 0.17$ & $-0.04 \pm 0.64$ & $-0.01 \pm 0.17$ & $-0.08 \pm 0.64$ & $-0.02 \pm 0.17$ \\
\hline NGC 5794 & $0.03 \pm 0.16$ & $0.01 \pm 0.05$ & $0.05 \pm 0.16$ & $0.01 \pm 0.05$ & $0.02 \pm 0.16$ & $0.00 \pm 0.05$ \\
\hline IC 1078 & $0.01 \pm 0.15$ & $0.00 \pm 0.03$ & $-0.00 \pm 0.15$ & $-0.00 \pm 0.03$ & $-0.01 \pm 0.15$ & $-0.00 \pm 0.03$ \\
\hline NGC 6977 & $0.06 \pm 0.22$ & $0.01 \pm 0.03$ & $-0.01 \pm 0.22$ & $-0.00 \pm 0.03$ & $-0.06 \pm 0.22$ & $-0.01 \pm 0.03$ \\
\hline SDSSJ015424 & $0.04 \pm 0.32$ & $0.01 \pm 0.12$ & $-0.03 \pm 0.32$ & $-0.01 \pm 0.12$ & $-0.06 \pm 0.32$ & $-0.02 \pm 0.12$ \\
\hline NGC 2691 & $0.02 \pm 0.44$ & $0.01 \pm 0.15$ & $0.01 \pm 0.44$ & $0.00 \pm 0.15$ & $-0.01 \pm 0.44$ & $-0.00 \pm 0.15$ \\
\hline NGC 2780 & $0.25 \pm 0.58$ & $0.02 \pm 0.05$ & $0.04 \pm 0.58$ & $0.00 \pm 0.05$ & $-0.21 \pm 0.58$ & $-0.02 \pm 0.05$ \\
\hline UGC 06517 & $0.12 \pm 0.22$ & $0.06 \pm 0.11$ & $-0.06 \pm 0.22$ & $-0.03 \pm 0.11$ & $-0.19 \pm 0.22$ & $-0.09 \pm 0.11$ \\
\hline NGC 5145 & $0.25 \pm 0.81$ & $0.09 \pm 0.31$ & $0.18 \pm 0.81$ & $0.07 \pm 0.31$ & $-0.07 \pm 0.81$ & $-0.03 \pm 0.31$ \\
\hline NGC 5950 & $0.02 \pm 0.07$ & $0.01 \pm 0.06$ & $0.01 \pm 0.07$ & $0.01 \pm 0.06$ & $-0.01 \pm 0.07$ & $-0.00 \pm 0.06$ \\
\hline UGC 10803 & $0.06 \pm 0.09$ & $0.05 \pm 0.07$ & $0.01 \pm 0.09$ & $0.01 \pm 0.07$ & $-0.06 \pm 0.09$ & $-0.04 \pm 0.07$ \\
\hline MCG-01-52-012 & $0.03 \pm 0.37$ & $0.05 \pm 0.53$ & $0.02 \pm 0.37$ & $0.03 \pm 0.53$ & $-0.01 \pm 0.37$ & $-0.02 \pm 0.53$ \\
\hline UGC 09837 & $0.06 \pm 0.11$ & $0.01 \pm 0.02$ & $-0.03 \pm 0.11$ & $-0.01 \pm 0.02$ & $-0.09 \pm 0.11$ & $-0.02 \pm 0.02$ \\
\hline UGC 12250 & $0.57 \pm 1.26$ & $0.04 \pm 0.08$ & $-0.27 \pm 1.26$ & $-0.02 \pm 0.08$ & $-0.84 \pm 1.26$ & $-0.06 \pm 0.08$ \\
\hline NGC 5947 & $-0.13 \pm 0.09$ & $-0.02 \pm 0.01$ & $-0.12 \pm 0.09$ & $-0.02 \pm 0.01$ & $0.00 \pm 0.09$ & $0.00 \pm 0.01$ \\
\hline
\end{tabular}

